\title{
Intelligent-Illumination STED
}

\author{
Dissertation \\ zur Erlangung des mathematisch-naturwissenschaftlichen Doktorgrades \\ „Doctor rerum naturalium“ \\ der Georg-August-Universität Göttingen \\ im Promotionsprogramm PROPHYS \\ der Georg-August University School of Science (GAUSS)
}

vorgelegt von

Jörn Heine

aus Gifhorn

Göttingen, 2017 


\section{Betreuungsausschuss}

Prof. Dr. Stefan W. Hell, Department of NanoBiophotonics, Max Planck Institute for Biophysical Chemistry, Göttingen

Prof. Dr. Tim Salditt, Institute for X-Ray Physics / Nanoscale Imaging of Cellular Dynamics, Georg-AugustUniversität Göttingen

Dr. Benjamin Harke, Abberior Instruments GmbH, Göttingen

\section{Mitglieder der Prüfungskommission}

Referent: Prof. Dr. Stefan W. Hell

Korreferent: Prof. Dr. Tim Salditt

\section{Weitere Mitglieder der Prüfungskommission}

PD Dr. Alexander Egner, Optical Nanoscopy, Laser-Laboratorium Göttingen

Prof. Dr. Stefan Klumpp, Department for non-linear Dynamics, GeorgAugust-Universität Göttingen

Prof. Dr. Sarah Köster, Institute for X-Ray Physics / Nanoscale Imaging of Cellular Dynamics, Georg-AugustUniversität Göttingen

Prof. Dr. Stefan Jacobs, Department of NanoBiophotonics, Max Planck Institute for Biophysical Chemistry, Göttingen

Tag der mündlichen Prüfung: 
We dance around in a ring and suppose,

But the Secret sits in the middle and knows.

Robert Lee Frost 



\section{Contents}

Abstract I

List of Abbreviations II

Disclaimer III

$\begin{array}{ll}\text { 1. General Introduction } & 1\end{array}$

1.1. Microscopy . . . . . . . . . . . . . . . . . . . . . . . 1

1.2. Superresolution microscopy . . . . . . . . . . . . . . . . 4

1.2.1. Coordinate-targeted methods . . . . . . . . . . . . 5

1.2.2. Coordinate-stochastic methods . . . . . . . . . . . . 5

1.3. STED fluorescence nanoscopy . . . . . . . . . . . . . . . . 6

1.4. Photobleaching in STED nanoscopy . . . . . . . . . . . . . . . 10

1.5. Motivation . . . . . . . . . . . . . . . . . 12

\begin{tabular}{|l|l|}
\hline 2. Fluorescence Photon Budget and STED Resolution & 13
\end{tabular}

2.1. Introduction . . . . . . . . . . . . . . . . . . . . . . . . . . 13

2.2. Fluorescence signal and resolution . . . . . . . . . . . . 15

2.3. Collecting the maximum fluorescence signal . . . . . . . . . . . . 19

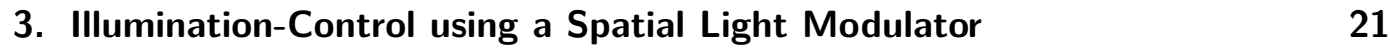

3.1. STED nanoscopy setup . . . . . . . . . . . . . . . . . . . 21

3.2. OFF-switching pattern enhancement . . . . . . . . . . . . . 24

3.2.1.2D-STED . . . . . . . . . . . . . . . . . 24

3.2.2. Axial-STED . . . . . . . . . . . . . . . 26

3.3. Axial-STED in live-cell embedded samples . . . . . . . . . . . . . . . . 28

3.3.1. Imaging depths for different objectives . . . . . . . . . . . . 29

3.3.2. Correction for spherical aberrations . . . . . . . . . 31

$3.3 .3 . \quad$ Live-cell imaging . . . . . . . . . . . . . . . . . . . . 33

\begin{tabular}{|l|} 
4. Simpler MINFIELD Illumination \\
\hline 5
\end{tabular}

4.1. Introduction . . . . . . . . . . . . . . . . . . . . . . . . . . . . . 35

4.2. MINFIELD with galvanometric scanners . . . . . . . . . . 36

4.3. 2D-STED MINFIELD . . . . . . . . . . . . . . . . . . 37

$4.4 . \quad 3 \mathrm{D}-\mathrm{STED}$ MINFIELD $\ldots \ldots \ldots \ldots$ 
4.5. MINFIELD in the green dye spectrum . . . . . . . . . . . . . 41

5. Adaptive-Illumination STED Nanoscopy 43

5.1. Introduction . . . . . . . . . . . . . . . . . 43

5.2. Concept of DyMIN . . . . . . . . . . . . . . . . . 44

5.3. Simulation framework . . . . . . . . . . . . . . . 48

5.4. Assessment of DyMIN . . . . . . . . . . . . . . . . . 50

5.4.1. Simulated light dose reduction for different samples. . . . . . 52

5.4.2. Experimental parameter optimization . . . . . . . . 55

5.5 .2 D-STED DyMIN applications $\ldots \ldots \ldots \ldots$. . . . . . . 58

5.5.1. Applying DyMIN to biological samples . . . . . . . . . . . . 58

$5.5 .2 . \quad$ Evaluation of the 2D-STED DyMIN resolution . . . . . . . 60

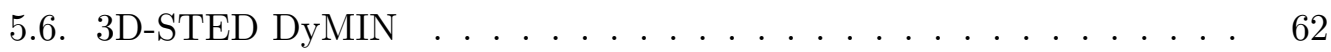

5.6.1. Experimental parameter optimization . . . . . . . . 62

5.6 .2$. Improved imaging of a volume . . . . . . . . . . . . 63

$5.6 .3 . \quad$ Evaluation of the 3D-STED DyMIN resolution . . . . . . . 65

5.7. Multi-color DyMIN imaging . . . . . . . . . . . . . . . . 66

5.8. Heterogeneous photobleaching and structure probing . . . . . . . . 68

\begin{tabular}{ll}
\hline 6. Conclusion and Outlook & $\mathbf{7 1}$
\end{tabular}

6.1. Summary . . . . . . . . . . . . . . . . . . . 71

6.2 . Improving conventional STED scanning . . . . . . . . . . . . . . . . 72

6.3. Intelligent-illumination schemata . . . . . . . . . . . . . . 74

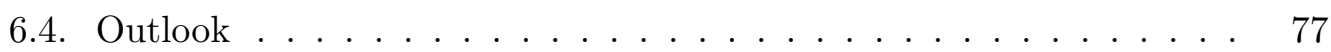

\begin{tabular}{lr}
\hline 7. Material and Methods & 81
\end{tabular}

7.1. General statements . . . . . . . . . . . . . . . . . . . . . . . . . . . . 81

7.2. Sample preparation . . . . . . . . . . . . . . . . 81

\begin{tabular}{lr}
\hline Bibliography & 85
\end{tabular}

\begin{tabular}{lr}
\hline A. Appendix & 99
\end{tabular}

A.1. Further information . . . . . . . . . . . . . . . . . . . . . 99

A.2. Imaging parameters . . . . . . . . . . . . . . . . . 104

\begin{tabular}{lr}
\hline Danksagungen & 115
\end{tabular} 


\begin{abstract}
Recently established fluorescence superresolution microscopy techniques, such as stimulated emission depletion (STED), are capable of imaging fixed and living cells at the nanometer scale. In STED, ON- and OFF-switching of fluorophores is performed with an excitation and a STED laser beam, respectively. During each switching cycle a finite probability exists that the fluorophore is photobleached. This hampers the possible STED resolution and sample structure definition.

In this thesis, the connection between photobleaching, the obtainable fluorescence signal and the STED resolution was investigated. It was found that the maximum fluorescence signal extractable from a sample is inversely proportional to the STED resolution increase to the power of four. As a reverse conclusion, a higher fluorescence signal will always facilitate a better STED resolution and sample structure representation.

To achieve such higher signals, a STED microscope was built which uses a spatial light modulator (SLM) as the phase mask element for the OFF-switching pattern creation. Especially under live-cell conditions, and at great sample depths, the SLM has conceptual advantages compared to conventional elements.

To push the maximum fluorescence signal and resolution further, novel intelligentillumination scan schemata were developed. Those locally reduce photobleaching in the sample. The MINFIELD scan scheme (sub-diffraction sized scan fields of $\sim 50-200 \mathrm{~nm}$ ) was realized with 2D- and 3D-STED using intelligent beam control, leading to a simple microscope design with a great application range.

The novel illumination scheme, Dynamic intensity MINimum (DyMIN), combines the advantages of MINFIELD and of the sample responsive illumination concept RESCue. With DyMIN, just as little OFF-switching intensity is applied to the current sample structure to have a clear ON/OFF-separation of the fluorophores. The typically much lower light dose provides a superior bleaching reduction for a large scan field. With 2D- and 3D-STED DyMIN scanning, a reduction of the light dose acting directly on the fluorophores up to 20 -fold was possible, and the overall illumination of the sample was lowered more than 100-fold for sparser samples, both compared to a similar conventional scan. DyMIN enabled the best ever reported STED resolutions for a large field of view, $\sim 17 \mathrm{~nm}$ in the lateral direction, and $\sim 34 \mathrm{~nm}$ in the axial direction.
\end{abstract}




\title{
List of Abbreviations
}

\author{
AFM ......... Atomic Force Microscopy \\ AOM........ Acousto-Optical Modulator \\ APD .......... Avalanche Photo Diode \\ AU $\ldots \ldots \ldots \ldots$ Airy Units \\ a.u. .......... Arbitrary Units \\ CW .......... Continuous Wave \\ DyMIN ....... Dynamic Intensity Minimum \\ FFT …...... Fast Fourier Transformation \\ FLIM $\ldots . . \ldots$. Fluorescence Lifetime Imaging \\ FPGA ….... Field Programmable Gate Array \\ FWHM ....... Full Width at Half Maximum \\ ISC $\ldots \ldots \ldots$ Intersystem Crossing \\ NA $\ldots \ldots \ldots$ Numerical Aperture \\ NPC $\ldots . . \ldots .$. Nuclear Pore Complex \\ PALM ........ Photoactivated Localization Microscopy \\ PSF .......... Point Spread Function \\ SEM .......... Scanning Electron Microscopy \\ SIM $\ldots . . . \ldots . .$. Structured Illumination Microscopy \\ SiR $\ldots . . . \ldots .$. Silicon Rhodamine \\ SLM ......... Spatial Light Modulator \\ STED ........ Stimulated Emission Depletion \\ STORM ...... Stochastic Optical Reconstruction Microscopy \\ TEM ….... Transmission Electron Microscopy \\ TIRF ........ Total Inner Reflection Fluorescence
}




\section{Disclaimer}

Parts of the here presented work are already published in peer-reviewed articles. These are: Fabian Göttfert et. al. (Strong signal increase in STED fluorescence microscopy by imaging regions of subdiffraction extent, 2017) [1]; Jörn Heine et. al (Adaptive-Illumination STED Nanoscopy, 2017) 2. With respect to the „Promotionsordnung" of July $2012 \S 10$ (2) of the Georg-August-University School of Science (GAUSS) in Göttingen I reuse parts of these publications (text, data, and figures) in this thesis. Partly the text and figures taken are modified and/or extended for enhanced clarity. 



\section{General Introduction}

In this chapter, an overview about microscopy in general, and superresolution microscopy techniques is given. Namely the coordinate-targeted methods and the coordinate-stochastic methods.

\subsection{Microscopy}

The ideal microscope captures all the processes, actions, structures and changes in a cell with an unlimited spatial and temporal resolution. Scientists can easily zoom in on a spot of interest and slow down processes in their observation to see every detail, or rewind things if necessary. The cellular life would be understood quickly. Unluckily, this microscope does not exist yet. Real microscopes can often only feature a small subset of the requirements, and most of the time a lot information is lost due to principle physical limitations such as the diffraction barrier.

However, even with all their limitations, microscopes are in general the main tool to study life on the cellular level, which is of course of great interest to mankind. The light microscope features one of the widest application ranges in this respect. It has a decent spatial and a good time resolution, and the sample preparation is relatively easy to perform. It can acquire images with multiple colors [3 7], and most important, it is capable of studying processes inside a living cell [8 11], since the light is minimally invasive and most cells are optically transparent.

However, when trying to image sub-cellular components with a light micoscope, the structure starts to blur at a certain feature level (size of the structure). In fact, no detail much smaller than approximately half the wavelength of light $(\sim 180 \mathrm{~nm})$ can be optically separated with a microscope. This problem, known as the diffraction barrier, was first reported by Ernst Abbe in 1873 [12], and for a long time was the absolute resolution limit for far-field light microscopy. The diffraction-limited resolution $d_{\text {min }}$, defined as the full width at half maximum (FWHM), in the lateral imaging plane $(x, y)$ is

$$
d_{x, y \min } \approx \frac{\lambda}{2 n \sin (\alpha)}
$$




\section{General Introduction}

and along the optical axis $(z)$

$$
d_{z \min } \approx \frac{\lambda}{n-\sqrt{n^{2}-n^{2} \sin ^{2}(\alpha)}}
$$

for a high numerical aperture (NA) [13]. $\lambda$ is the wavelength of light, $n$ the refractive index of the medium between objective lens and sample, and $\alpha$ the half opening angle of the light focus. The NA is the product of $n$ and $\sin (\alpha)$. When using light at a wavelength of $500 \mathrm{~nm}$, and an objective lens with an NA of 1.4, the resolution is almost at its maximum with $\sim 180 \mathrm{~nm}$ laterally and $\sim 540 \mathrm{~nm}$ axially. Better resolutions can hardly be achieved with conventional methods, because in practice a much shorter wavelength cannot be used. The reason is that at shorter wavelengths, light starts to be toxic to cells [14], and that the glass lenses absorb the light below a certain wavelength. Another limit occurs from the NA which has a maximum at $n$ (typically $n \approx 1.518$ ) times the sine of $\alpha=90^{\circ}$. Luckily, the resolution barrier was fundamentally broken in the late nineties by superresolution microscopy [15 18], see the following section 1.2 .

Besides superresolution microscopy, several microscopy techniques exist which feature a higher spatial resolution compared to conventional light microscopy.

Very high spatial information can be collected when using accelerated electrons instead of light to probe the sample. The Electrons have a wavelength which is orders of magnitudes lower compared to light. Electron microscopes can therefore achieve resolutions on a molecular or even atomic scale. Two basic configurations for electron microscopes exist, the scanning electron microscope (SEM) [19] and the transmission electron microscope (TEM) 20. In SEM, a focused electron beam is scanned over the sample, and the scattered electrons are detected as the signal. In TEM, a very thin sample is homogeneously irradiated, and the transmitted electrons are detected behind the sample. For both electron microscope techniques the drawback is that the sample has to be imaged in a vacuum, otherwise the surrounding gas would absorb the electrons. Live-cell experiments with electron microscopy are hence impossible. Additionally, in SEM only information from the sample surface can be collected since the electrons are scattered at the first material they arrive at. To gain information from inside a cell (and even in three dimensions), the cell is frozen and then cut into thin layers. Each layer is individually imaged and afterwards all images are fused to form a volume 21. A combination of SEM and fluorescence microscopy can provide structural and functional information [22].

Atomic force microscopy (AFM) uses a thin „needle“to directly „touch"the sample and extract information from the near-field. The needle is moved over the sample surface and is continuously adapted to the surface height. By that, the sample surface topography is recorded with a resolution higher compared to far-field light 
microscopy 2325$]$.

In confocal laser scanning microscopy, the sample is scanned with a focused laser spot which is diffraction-limited [26]. Specific sample structures are typically labeled with fluorescent dyes or proteins (immunofluorescence 27,29 which are excited by the laser spot. The resulting fluorescence light from the labels is focused through a pinhole which is in a conjugated plane to the sample. Light which originates from out-of-focus sample planes is blocked at the pinhole to a large extent, whereby the contrast of the image is enhanced. With small pinhole sizes also the resolution of the microscope is improved. For an infinitely small pinhole size the resolution is enhanced by factor of $\sqrt{2}[13$, but no light is transmitted. A backprojected pinhole size in the sample of 0.5 - 1.0 Airy Units (AU) is a typical compromise between resolution enhancement and light transmission. Recently developed methods, namely Airyscan [30, 31] and rescan-microscopy [32, feature the maximum confocal resolution improvement while detecting most of the fluorescence signal. Both techniques use an array detector to record the fluorescence light and reassign the spatially distributed signal to the imaged pixel.

In a laser scanning microscope, a very short and intense laser pulse at a lower photon energy (longer wavelength compared to single-photon excitation) can be used for two-photon excitation [33. Because the excitation probability has a quadratic dependency on the excitation intensity (which is Gaussian-shaped in the focus), the resolution is increased by a factor of $\sqrt{2}$. Nevertheless, since (typically) a doubled excitation wavelength is used, the two-photon resolution is in fact worse. The advantages of two-photon excitation are a deeper penetration depth and lower scattering. Additionally, photobleaching occurs only close to the focal spot and out of focus sample planes remain nearly unaffected.

A good axial resolution, but only at the coverslip surface, can be attained with total internal reflection fluorescence microscopy (TIRF) 34. For that, illumination light is focused to the outer region of the back aperture of a high numerical aperture objective lens (e.g. NA of $\approx 1.49$ ). Collimated light leaves the objective lens with an illumination angle in respect to the sample, which is larger than the critical angle for total internal reflection at the surface between coverslip and sample mounting medium. A resulting evanescent field penetrates (illuminates) the sample only $100-200 \mathrm{~nm}$ deep, depending on the illumination angle.

A way to double the axial and lateral resolution is structured illumination microscopy (SIM) 35 37. For this method, the sample is illuminated with a sinusoidal pattern which is rotated and shifted in the sample plane. The light pattern (with a maximum frequency dictated by diffraction) interacts with the spatial frequencies of the sample. Thereby Moiré fringes arise, which are detected in a widefield configuration. The information of each pattern position is fused in the Fourier space. The illumination pattern frequency is filtered, and back transformation to the real space produces an image of the sample with doubled resolution. A higher resolution can 


\section{General Introduction}

be achieved by saturating the excitation $[38$. In practice, photobleaching increases with the excitation saturation, due to the high intensities at the dye absorption maximum. This limits the achievable resolution with saturated structured illumination.

In a $4 \mathrm{Pi}$ microscope the $\mathrm{NA}$ is increased by coherently overlaying the focus spots of two opposite objective lenses (e.g. one above and one below the sample). Due to the higher NA, an almost complete spherical wavefront is used in a confocal laser scanning microscope-like arrangement. An optical axial resolution down to $75 \mathrm{~nm}$ was reported [39]. However, the point spread function (PSF) of a 4Pi microscope has strong sidelopes, which disturb the image. These can be sufficiently suppressed by two-photon excitation 40 .

\subsection{Superresolution microscopy}

Superresolution microscopy became very important in the last years [16 [41. This was mainly because it enables imaging living biological samples with multiple colors and at a resolution of a few tens of nanometers, which is well beyond the classical optical diffraction limit.

For superresolution microscopy marker molecules are used which label a particular structure [27, 42, 43]. If the marker molecules are spaced closer than the diffraction limit, they would normally fluoresce simultaneously (the smallest possible excited spot is diffraction-limited), and the recorded fluorescence signal of each marker would also be overlapped by light diffraction. Hence it is impossible to separate or locate the individual molecules. In superresolution microscopy the markers can sequentially be switched between an ON-state (fluorescent) and an OFF-state (nonfluorescent) [16]. The principle of superresolution microscopy is to keep a large number of the marker molecules in the OFF-state while only a small subset is in the ON-state. The resulting signal which originates from the ON-fraction is no longer overlapped by all the other signals and can be assigned to a specific point in the sample. In a next step, the ON-molecules are switched OFF and another small fraction of molecules is transferred into the ON-state which then readout. To acquire a complete superresolved image, this procedure is continued until every sample position (marker molecule) of the sample was readout.

To date, two basic concepts exist for superresolution microscopy, the coordinatetargeted method (see section 1.2.1) and the coordinate-stochastic method (see section 1.2.2). For both concepts, different configurations have been developed 444 45. Very recently a method was reported which combines the stochastic marker activation with a coordinate-targeted readout of a single activated molecule, whereby a nanometer localization precision of the molecule was shown [46]. 


\subsubsection{Coordinate-targeted methods}

For coordinate-targeted superresolution, a small area of the sample is typically prepared in the ON-state by a focused Gaussian laser beam. Thereby the area cannot be made endlessly small because the laser focus for preparation is diffraction-limited. After the ON-preparation, a second laser beam is focused to a pattern which (typically) switches OFF the outer region of the ON-area again. The resulting fluorescent spot is smaller, the resolution is increased. Thereby the OFF-switching works in a statistical way, the remaining ON-marker density follows roughly an exponential decay depending on the OFF-switching light intensity and cross-section for OFFswitching [47].

Different OFF-switching patterns can be used which usually feature an intensity minimum (zero) in the respective centers [48]. At these minimum positions the molecules remain in the ON-state. A little away from the minimum the intensity of the OFF-switching light pattern rises and some marker molecules are transferred into the OFF-state, following the exponential decay. At a certain distance to the minimum, the OFF-switching pattern intensity reaches a level where effectively every marker molecule is switched OFF (saturated). Since the maximum OFFswitching pattern steepness (frequency) is again dictated by the diffraction limit, the only way to reach the saturated point closer to the minimum (leading to a higher resolution), is to increase the overall intensity of the OFF-switching illumination. In principle, the resolution can be unlimited for high OFF-switching intensities. In practice, those high intensities cannot be used, mainly because they induce photobleaching [1] [49].

\subsubsection{Coordinate-stochastic methods}

For coordinate-stochastic superresolution (also known as single molecule microscopy) all marker molecules are typically prepared in the OFF-state. Now a small subset of all markers is switched into the $\mathrm{ON}$-state, which happens randomly in space and over the full field of view [16. Thereby it is crucial that all ON-markers have a distance in respect to each other which allows their clear separation in the detected image. The ON-markers are continuously illuminated and imaged, and the fluorescence signal of each marker is accumulated. Every marker produces a diffraction-limited blurred spot in the image. Since every spot corresponds to only one marker - as defined the marker position can be localized with a precision higher as the diffraction limit. The precision of the localization mainly depends on the square root of the number of photons collected for a marker [50]. Now, the already imaged ON-markers are transferred into an OFF-state. The next subset of ON-markers is prepared and imaged, and so on, until all markers have been recorded. In principle, the resolution 


\section{General Introduction}

can be unlimited for an infinite high number of photons collected. In practice, the marker molecules unwanted switch to an OFF-state (e.g. bleaching or blinking) during illumination, limiting the maximum number of fluorescence photons collected and hence localization precision for each marker.

The first reported coordinate-stochastic method is photoactivated localization microscopy (PALM) [50] [51. In its first implementation a fluorescent protein was used which is per default in the OFF-state. A sparse number of the proteins was activated with a brief laser pulse (e.g. $\left.\lambda_{a c t}=405 \mathrm{~nm}\right)$ and imaged with (e.g. $\left.\lambda_{e x c}=561 \mathrm{~nm}\right)$ to the point where most of the fluorescent probes were bleached. Again, a subset was activated, and so on, until all inactivated unbleached molecules were imaged.

A second method uses a photo-switchable fluorophore that can be switched between an ON- and OFF-state by two different laser wavelengths, and is called stochastic optical reconstruction microscopy (STORM) 52 [53. Typically, a strong red laser pulse prepares all fluorophores in the OFF-state. A green laser switches ON a little subset of the fluorophores for imaging until those are either switched OFF again by the (red) excitation illumination or until they bleach.

For axial resolution enhencement, artificial astigmatism can be applied in the detection path of the microscope. Thereby each detected blurred spot (corresponding to one ON-fluorophore) has an elliptical shape, depending on the axial position of the fluorophore in the sample. The $z$-position of the marker is encoded in the direction and length of the long elliptical axis [54 [55].

\subsection{STED fluorescence nanoscopy}

The first superresolution technique ever reported is stimulated emission depletion (STED) [15]. This coordinate-targeted method is basically a confocal laser scanning microscope with an additional STED laser for OFF-switching of marker molecules. In STED, a laser pulse excites the molecules in a diffraction-limited volume to a high vibrational singlet state $\mathrm{S}_{1}$, vib. Due to very fast molecular internal loss of the vibrational energy, the molecule transitions into a low vibrational excited state $\mathrm{S}_{1}$ (see also figure 1.2 in section 1.4). From this energy level (after a certain state lifetime), a marker would normally spontaneously fall to any vibrational ground state $\mathrm{S}_{0}$, whereby it would emit a red-shifted fluorescence photon with respect to the excitation wavelength (spontaneous emission). In STED microscopy this is prevented by an intense (pulsed) STED laser with a wavelength at the red tail of the marker emission spectrum. The STED light quickly (before spontaneous emission could occure) forces the excited marker down to a high vibrational ground state $\mathrm{S}_{0 \text {, vib }}$ by stimulated emission. The population of this high vibrational ground state again decays very fast, and the STED laser photon energy is too low to overcome 
the energy gap between $S_{1}$ and $S_{0}$. Therefore the probability of re-excitation after stimulated emission to the $\mathrm{S}_{1}$ state by the STED laser light is low.

The STED and excitation wavelengths are blocked in the detection to avoid imaging of beam reflections and to exclude the low-resolution photons that arise from the stimulated emission. Only the fluorescence photons with a wavelength which is between the excitation and STED beam wavelengths are detected. These photons can only originate from the small vicinity of the STED OFF-switching minimum. Meaning the effective detected fluorescence spot is smaller compared to the confocal counterpart, the resolution is increased.

Several OFF-switching patterns exist, but only two are commonly used for STED microscopy. Both are shown in figure 1.1 .
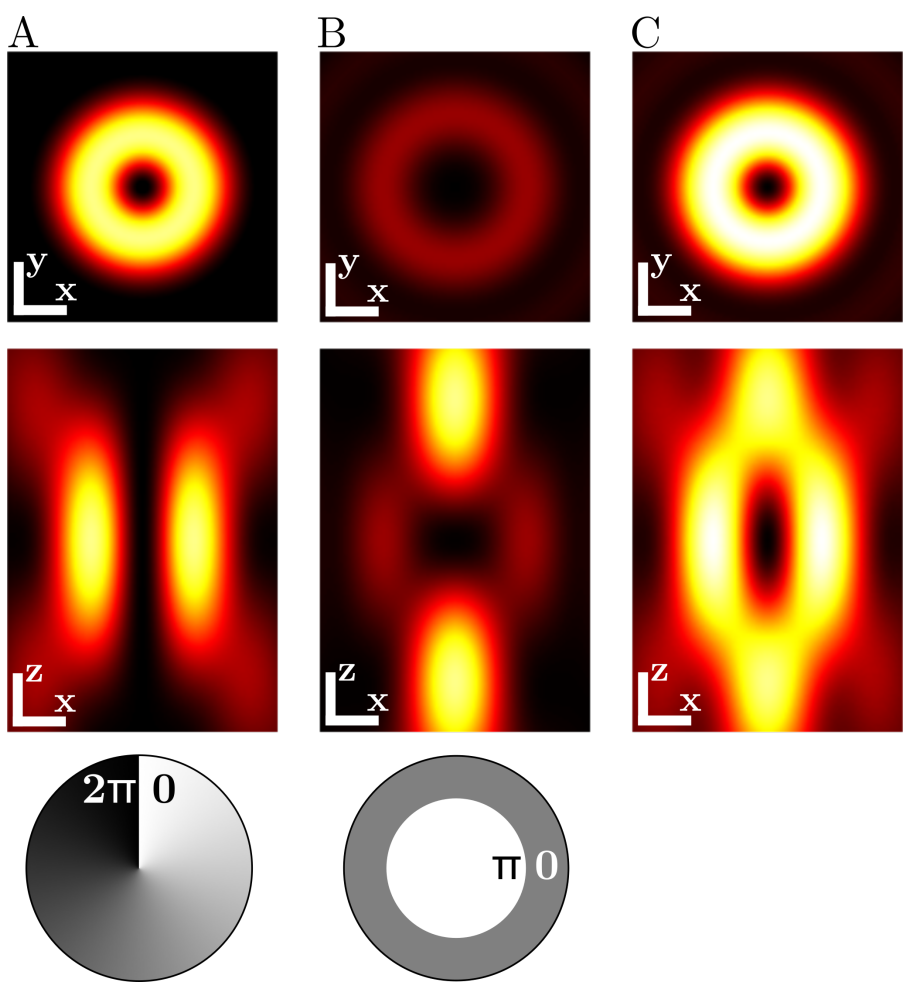

Figure 1.1.: Simulated intensity distributions for different STED modes and corresponding phase masks. Simulations for an NA of 1.4 and a STED wavelength of $775 \mathrm{~nm}$. OFF-switching patterns in $x y$-view (top), $x z$-view (middle), and corresponding phase masks (bottom). (A) 2D-STED, (B) axial-STED. For 2DSTED a helical phase ramp from $0-2 \pi$ is used (A, bottom). The phase mask for axial-STED has a concentric inner circle phase shifted by $\pi$ (B, bottom). (C) 3DSTED, an incoherent combination of both patterns of (A) and (B). Look-up table is the same for each OFF-switching pattern view. Scale bars $(x, y),(x, z)$ are $200 \mathrm{~nm}$. 


\section{General Introduction}

The best lateral resolution improvement ( $x$ - and $y$-directions in the sample) is achievable with the doughnut-shaped OFF-switching pattern in figure 1.1 (A), namely 2D-STED. This pattern is generated when a circular polarized wavefront with a helical phase ramp of $2 \pi$ (Figure 1.1 (A), bottom) is focused. Opposite points of the wavefront always have a phase shift of $\pi$, whereby every pair interferes destructively at the optical axis. The pattern has an quadratic course close to the focus spot in any lateral direction. However, this pattern only features a lateral resolution improvement while the axial resolution is the same as for a confocal laser scanning microscope. A better resolution along the optical axis ( $z$-direction), and also a little in the lateral plane, can be realized with the OFF-switching pattern shown in figure 1.1 (B), namely axial-STED. This pattern is generated by a focused wavefront with a central circle phase delay of $\pi$ (Figure 1.1 (B), bottom). The diameter of the inner circle is roughly $1 / \sqrt{2}$ of the back aperture diameter $d_{B A}$ of the used objective lens [48]. The axial-STED pattern has approximatly a quadratic intensity course along $z$, but only a $x^{4}, y^{4}$ course along the lateral directions, both close to the focal spot. A quadratic course in each direction would be preferred, since it features the best STED resolution at a certain OFF-switching power. Unluckily, no OFF-switching pattern exists which is able to feature a quadratic course in each spatial direction with a single beam polarization [56. However, a quadratic course in each direction is possible, if two OFF-switching patterns are overlayed incoherently (e.g. the 2D- and axial-STED pattern), shown in figure 1.1 (C). This OFF-switching pattern combination is called 3D-STED. By changing the power of both individual patterns, the resolution can be tuned independenly in the lateral and axial directions 57,59 .

The 2D-STED resolution (in the lateral plane) follows

$$
d_{x, y \min } \approx \frac{\lambda}{2 n \sin (\alpha) \sqrt{\mathbf{1}+\frac{\boldsymbol{I}}{\boldsymbol{I}_{\boldsymbol{s}}}}}
$$

where the additional term $\sqrt{1+I / I_{s}}$ in the denominator compared to equation 1.1 takes the resolution increase (due to the OFF-switching) into account. $I$ is the intensity of the STED light, and $I_{s}$ is defined as the STED intensity where half of the initial ON-markers are switched OFF. In this thesis, the power of the STED beam $P$ in the focus point is used instead of the intensity, mainly because the power is easier to measure in contrast to the intensity. The saturation power $P_{s}$ (similar defined as $I_{s}$ ) is the STED power where the microscope resolution is increased by $\sqrt{2}$. However, $P_{s}$ is a microscope dependent parameter which can vary between different microscopes used. 
To calculate the axial-STED resolution, the same STED term applies in the denominator:

$$
d_{z \min } \approx \frac{\lambda}{n-\sqrt{n^{2}-n^{2} \sin ^{2}(\alpha)} \cdot \sqrt{\mathbf{1}+\frac{\boldsymbol{I}}{\boldsymbol{I}_{s}}}}
$$

For both, 2D-STED and axial-STED, the approximate square root dependence on the STED intensity originates from the quadratic course of both OFF-switching patterns in the respective directions [47. To calculate the lateral and axial resolution for 3D-STED, the respective resolutions for the 2D-STED and axial-STED pattern need to be used.

Several combinations of STED with other microscopy techniques were developed, as well as improvements for STED microscopy in general. For instance, the singlephoton excitation was replaced by a two-photon excitation to image deep inside (living) specimens 60,61. Fluorescence lifetime imaging microscopy (FLIM) is straight forward to combine with STED [5]. STED allows fluorescence correlation spectroscopy (FCS) with a higher spatial resolution [62 64]. An important improvement for STED was the invention of gating [65], which increases the final image resolution and contrast. For gating, the early fluorescence photons of the dye lifetime are discarded. At this early point in time, the STED laser has not switched OFF all marker molecules yet due to his finite pulse length, which would have been switched $\mathrm{OFF}$ at a later point of time (higher resolution). Only the fluorescence photons carrying the maximum resolution information, originating when the STED pulse is finished, are used. Before the nowadays used pulsed STED lasers were employed, continuous wave (CW) STED lasers were used in the visible dye spectrum 66 67. For those CW-STED approaches gating results in a fundamental resolution improvement. Here, because of the lower STED intensity, a longer OFF-switching time is required, and a lot of (early) low resolution fluorescence photons are present. Therefore a lot of the fluorescence photons are discarded in gated CW-STED. As a result, the signal level is relatively low compared to microscopes with a pulsed STED laser. Hence, the structure definition is worse as well as the maximum attainable resolution.

STED can also be combined with AFM to capture functional and topographic information of a cell [68] 69. The best isotropic STED resolution $(<35 \mathrm{~nm})$ was achieved by combining STED with 4Pi microscopy [70 [71]. To guarantee a permanent overlay of the excitation focus and 2D-STED OFF-switching pattern in a regular setup, a special phase mask element can be used. Without the best 


\section{General Introduction}

overlay of both beams, the fluorescence signal decreases. To achieve a coaligned overlay, both beams pass the same optical fiber, optical elements in the microscope, and the phase mask element. The element is designed such that the phase of the excitation wavefront is nearly untouched, while the STED wavefront experiences a phase information that forms a useful OFF-switching pattern for a lateral resolution increase [72].

To improve the imaging speed, attempts to parallelize the STED imaging process were made. In one attempt the excitation and STED beam were splitted to four independent focal spots, and merged the four resulting images to one $[73$. In another attempt, two orthogonal standing sinusoidal waves were used which were shifted in the focal plane. A reconstruction of the individual spatial fluorescence distributions, corresponding to different wave positions, generates a superresolved image [74. However, video rate time resolution is already realizable with a single STED focus within a small scan area 75$]$ [76.

Biological reasearch often requires to label different sample structures with differently colored dyes. Several ways for multicolor STED imaging exist: e.g. using two independent excitation and STED beams [6] different excitation beams with a single STED beam [59,78,79], or up to four colors separated only in the detection [7] [80. Three-color live-cell STED with three independent excitation wavelengths and detection windows using a single STED wavelength was recently reported. In this case, sample structure was partly labeled with long-stoke dyes to have a greater wavelength range to use compared to regular dyes 29.

\subsection{Photobleaching in STED nanoscopy}

Photobleaching is the irreversible transition of a fluorophore to a non-fluorescent state caused by illumination with photons. When a fluorophore is bleached before being imaged, it cannot contribute to the sample structure definition. In the presence of strong photobleaching, the STED image has a low image quality.

In superresolution microscopy typically fluorophores are used which emit a fluorescence photon when falling from the excited $S_{1}$ singlet state to the $\mathrm{S}_{0}$ ground state (Figure 1.2).

As soon as the molecule is excited, different energy pathways are possible which are connected to fluorescence signal loss or bleaching. The precise process is often hard to decode 82 , because it depends on many different parameters like the specific dye, environmental conditions, the wavelengths involved and their respective irradiation levels 83. For some dyes, detailed bleaching measurements were performed with green- and red-shifted irradiation in respect to the excitation. By illuminating the dye with the green-shifted photons, intentionally higher excited states were 


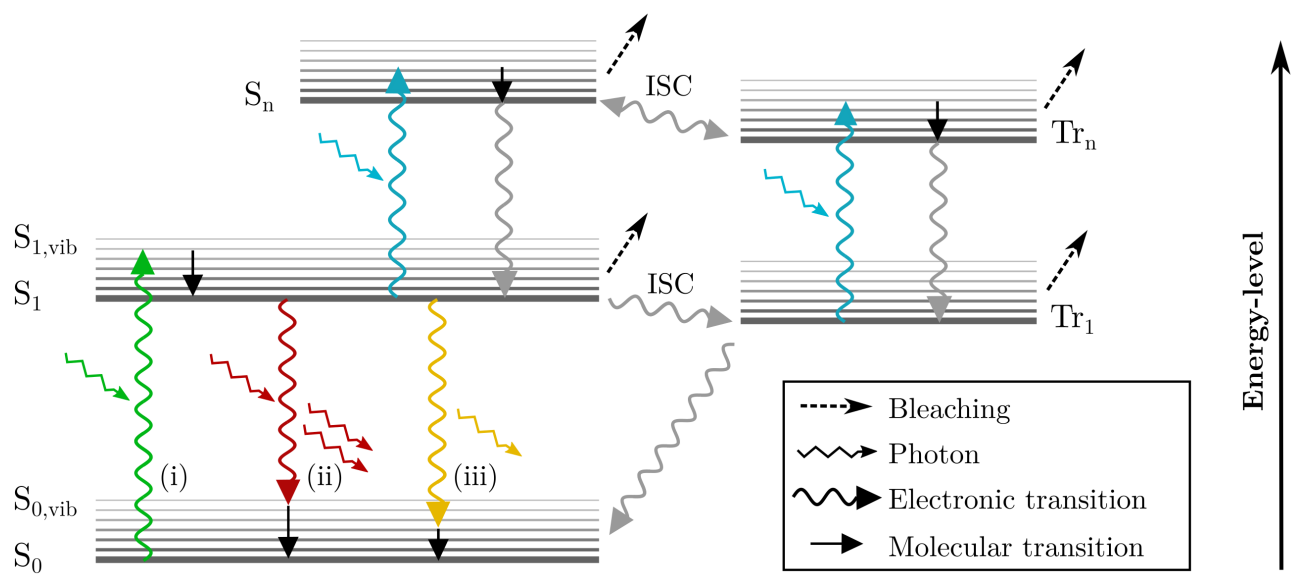

Figure 1.2.: Jablonski diagram of dye energy-levels. Modeled are the relevant valence electron singlet $(\mathrm{S})$ and triplet $(\mathrm{Tr})$ energy-levels, which are broadened to quasi-continuous energy bands due to vibrational and rotational molecular states. At room temperature, the relevant electron is in the ground state $\mathrm{S}_{0}$. By excitation with a photon, the electron can be transitioned to the $\mathrm{S}_{1}$ singlet state (i). From there, it maybe transitions to a higher singlet state $\left(\mathrm{S}_{\mathrm{n}}\right)$ by another photon-excitation, it transitions to a triplet state $\left(\operatorname{Tr}_{1} ; \operatorname{Tr}_{n}\right)$ by intersystem crossing (ISC), or returns to the ground state by either stimulated emission (ii) or spontaneous emission (iii). In this model, photobleaching may occur from any excitated state 81 .

populated which directly leads to a significantly higher photobleaching since more bleaching pathways are opened 84 . With the red-shifted photons, attempts were made to retrieve dyes from any triplet state $(\operatorname{Tr})$ to a singlet state $(\mathrm{S})$ [85]. A recovery from any triplet state (especially the $\operatorname{Tr}_{1}$ ) is preferable, because these are suspected to be the main bleaching pathways 86 [87. A strong reduction of photobleaching was observed when allowing relaxation from the triplet state $\left(\operatorname{Tr}_{1}\right)$ back to the ground state $\mathrm{S}_{0}$. This was achieved by using low repetition rates of the pulsed excitation in confocal and STED microscopy. Typically, an illumination pause between two pulses of more than 1 us was maintained. A different approach to reduce photobleaching is the use of a special mounting medium which quenches the $\operatorname{Tr}_{1}$ state by an included oxidizing or reducing compound [49] 88 .

In STED microscopy a short STED pulse can lead to two-photon excitation events, which excites the dye to higher energy levels as the $\mathrm{S}_{0}$ state [89]. This increases photobleaching since different bleaching pathways are possible from the higher excited states. However, avoiding two-photon excitation by using longer STED pulses (>150 ps) significantly reduces this effect [89] [90]. The STED wavelength may not only deplete the $\mathrm{S}_{1}$ population, but also excites the dye to any higher state from the $\mathrm{S}_{1}$ energy-level. Nevertheless, measurements on many different dyes showed, the dominant process is the intended de-excitation of the $\mathrm{S}_{1}$ state by stimulated emission 91 . 


\section{General Introduction}

In this thesis the fluorophore ATTO 647N (ATTO-TEC, Siegen, Germany) is frequently used. For this specific dye it was shown that photobleaching is mainly driven by STED photons in a linear dependency 1 .

\subsection{Motivation}

STED is basically an improvement of the well-established confocal microscope with a diffraction-free resolution. It therefore features a wide range of applications and the experiments are relatively easy to perform. Compared to other microscopy techniques with a resolution higher than the diffraction barrier, the STED principle works without any need for reconstruction or calculation of the image. Therefore it is known to produce almost artifact-free images of samples, capturing the reality closely.

Although STED microscopy became simpler and easier during the last twenty years, a lot of room for further improvements is present. The sample structure definition and resolution in STED microscopy is limited by the amount of fluorescence photons a dye molecule can contribute to the image before it is irreversibly bleached. Tools to increase the fluorescence signal, for instance by reducing the bleaching light dose, will help to acquire STED images with a higher resolution and with well defined structures. 


\section{Fluorescence Photon Budget and STED Resolution}

In this chapter, a link between the maximum fluorescence signal which can be collected in a STED image in dependence on the STED resolution is presented.

\subsection{Introduction}

In principle, STED can feature molecular resolution since only the intensity $I$ of the STED beam and the STED saturation intensity $I_{s}$ determine the resolution $\left(d_{\min } \propto 1 / \sqrt{I / I_{s}}\right)$, when $I$ becomes very large compared to $I_{s}$. Theoretically, increasing the STED intensity further and further would continuously lead to higher resolutions. However, due to the square root dependence on $I$, the resolution increases much slower at high resolutions while applying more STED power. To achieve a resolution of $1 \mathrm{~nm}$ with the microscope used in this thesis and common dyes, a STED power of more than $100000 \mathrm{~W}$ would be necessary in the sample. Up to now, no STED laser exists which features such high powers. Even if it did, such a molecular resolution is out of reach because of the loss of fluorescence signal due to photobleaching caused by the high irradiation. However, even at much lower light doses and resulting photobleaching, the fluorescence signal drops to a level where the structure is drowned in noise, hence the image is useless. Consequently, the optimization of the fluorescence signal will also provide the possibility to achieve the best resolution. In table 2.1 the most important effects which lower the fluorescence signal are listed, together with the main factors influencing them.

The quality (deepness) of the STED OFF-switching minimum (ideally zero) has a direct impact onto the STED image signal [47 [92]. When some light remains in the minimum, fluorescence is also de-excited (OFF-switching by the STED light) in this area, where it ideally should not be switched OFF. Unluckily, the effect of de-excitation by stimulated emission is very efficient at the beginning (low STED power), since it follows a square root dependence on the STED intensity. This means that already a little STED light in the minimum results in a relatively large fluorescence signal loss. Avoiding an unwanted filling of the STED minimum is hence of great benefit in terms of fluorescence signal. To optimize the OFF-switching 


\section{Fluorescence Photon Budget and STED Resolution}

Table 2.1.: Effects which lower the obtainable signal in a STED image.

\begin{tabular}{|c|c|}
\hline Basic effect & Influences \\
\hline $\begin{array}{l}\text { Quality of the OFF-switching } \\
\text { minimum }\end{array}$ & $\begin{array}{l}\text { Aberrations } \\
\text { Polarization of the STED beam } \\
\text { Inhomogeneous STED wavefront }\end{array}$ \\
\hline $\begin{array}{l}\text { Overlay excitation, STED minimum } \\
\text { and detection pinhole }\end{array}$ & $\begin{array}{l}\text { Basic Alignment } \\
\text { Drift between beams }\end{array}$ \\
\hline Fluorescence loss & $\begin{array}{l}\text { Bleaching of the dye molecules } \\
\text { Dark states }\end{array}$ \\
\hline
\end{tabular}

minimum, a spatial light modulator can be used, as described in chapter 3

With an ideal STED microscope (featuring a zero minimum for the OFF-switching pattern with diffraction-limited steepness, and a perfect alignment of all beams and detection), a maximum fluorescence signal can be achieved for a certain resolution, which is dictated by the photostability of the dye and the initial fluorescence level (number of dye molecules present). With a less photostable dye, the fluorescence signal can be integrated only for a shorter time period to arrive at the same level of bleaching. When less dye molecules are present, fewer fluorescence photons will be emitted.

When trying to obtain a higher spatial resolution by applying a higher STED power, the gain in resolution will cost fluorescence signal because of higher photobleaching induced by the higher STED intensity. At a certain amount of bleaching, the structure is eventually lost in noise, since photobleaching prevents collecting enough fluorescence signal for a structure representation. 


\subsection{Fluorescence signal and resolution}

A STED resolution increase leads to a lower fluorescence signal because of higher photobleaching. To derive the link between STED resolution and the maximum fluorescence signal, the following assumptions apply.

The pixel size is much smaller compared to the size of the excitation and OFFswitching intensity distributions in the focus. In the middle area of the linear scan (steady-state conditions), each pixel experiences the very same total light dose that drives photobleaching (excitation and OFF-switching illumination). Before a specific pixel is readout, half of the total bleaching dose has already been applied to the pixel during the imaging of earlier pixels. The same light dose is applied to the pixel after readout (Figure 2.1 (A)).

Assuming an exponential loss of the fluorescence level due to photobleaching (Fig-
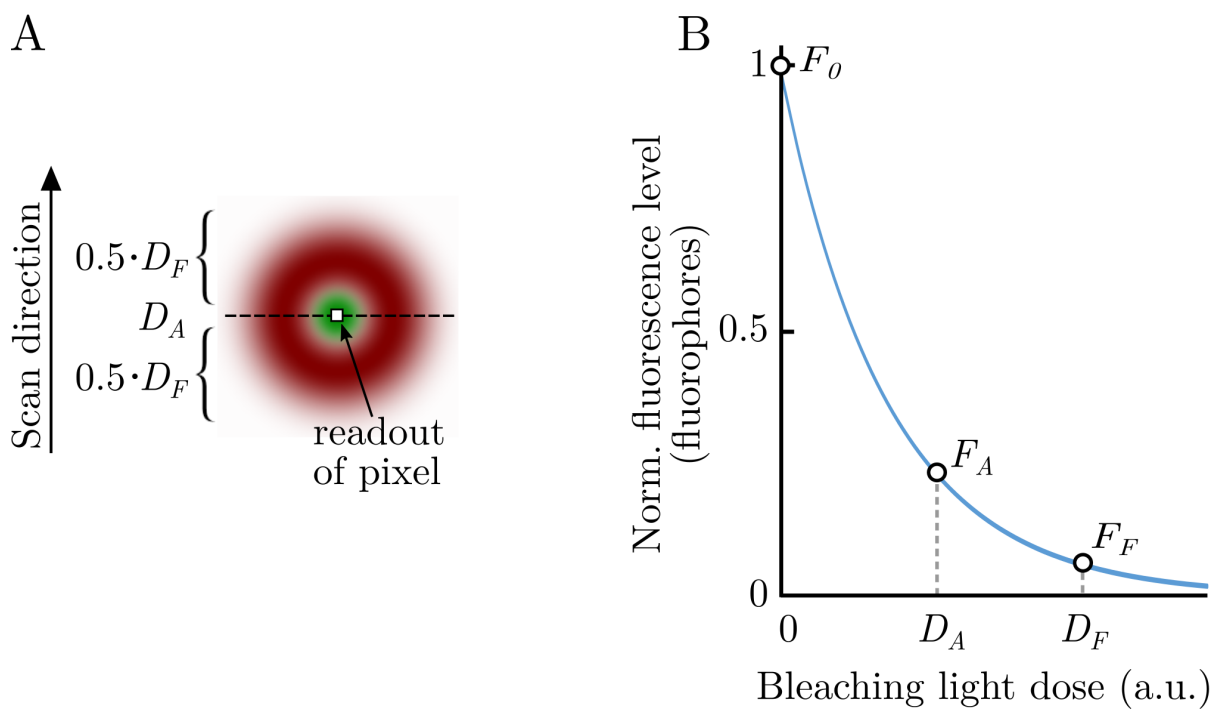

Figure 2.1.: Bleaching of the fluorescence level in STED imaging. (A) At steady-state conditions, in a nanoscope with point-scanning illumination, half of the bleaching light dose is applied at a certain pixel before the readout is performed. $D_{F}$ is the dosage after the scan, and $D_{A}=0.5 \cdot D_{F}$ is the dosage a pixel experiences before and after readout. (B) The fluorescence level during the readout is $F_{A}$ and the level after the linear scan is completed is $F_{F}$. Both levels are defined by an exponential photobleaching. Figure reproduced from ref. [2].

ure 2.1 (B)), the remaining fluorescence levels $F_{A}$ and $F_{F}$ are present during readout and after both illumination foci have passed, respectively. The fluorescence signal at a pixel is the product of the dwell time and the respective fluorescence level $F_{A}$ in 


\section{Fluorescence Photon Budget and STED Resolution}

the pixel, whereby $F_{A}$ decreases non-linearly with the exponential photobleaching course. At a low bleaching light dose (e.g. short dwell times), the fluorescence signal increases linearly with the dwell time. However, also the bleaching light dose increases linearly with the dwell time, whereby the fluorescence level decreases due to photobleaching. At a certain (best) dwell time the fluorescence signal is at a maximum. A longer dwell time decreases the fluorescence level non-linearly which is not compensated by the longer linear integration of fluorescence photons, thus the fluorescence signal starts to fall.

To derive the best dwell time, the following considerations apply: a fluorophore undergoes a certain amount of excitation and de-excitation events (cycles) while it is scanned. During each cycle, a finite probability exists that the fluorophore will transitions into a dark-state without recovery (photobleaching). For simplicity, bleaching is primarily introduced linearely with the STED illumination, as shown for the red dye ATTO 647N (ATTO-TEC, Germany) [1. The density function of the fluorophores present in the sample in respect to the photobleaching light dose is an exponential distribution for many fluorophores (continuous limit). The fluorescence signal $S_{f l}$ that can be collected from a sample scales linearly with the start fluorescence level $F_{0}$, which is the initial number of fluorophores times the amount of fluorescence photons they emit. $S_{f l}$ has a maximum $S_{f l, \max }$ at a certain dwell time. As mentioned before, this maximum is at the dwell time where a longer linear integration time cannot outperform the lower fluorescence level caused by the non-linear photobleaching which results from the longer illumination. To find the best dwell time (giving the maximum fluorescence signal), the number of photons leading to a photobleaching event $\sum N_{p h}$ at a certain pixel before readout happens needs to be calculated:

$$
\sum N_{p h}=t_{i m g} \cdot I \cdot \sigma \cdot \frac{1}{E_{p h}} \cdot \frac{n_{p x}}{2}
$$

where $t_{i m g}$ is the dwell time, $\sigma$ is a bleaching cross section per STED photon, $E_{p h}$ is the STED photon energy, and $n_{p x}=A / a^{2}$ is the number of pixels where photobleaching is driven. With $a$ as the pixelsize, and $A$ as the area of the focus where bleaching occurs.

The fluorescence signal is the product of the dwell time, a fluorescence photoncollecting efficiency $\eta$, and the start fluorescence level which decreases exponentially with the photobleaching events:

$$
S_{f l}=t_{i m g} \cdot \eta F_{0} \cdot e^{-C \cdot \sum N_{p h}}=t_{i m g} \cdot \eta F_{0} \cdot e^{\frac{-C \cdot t_{i m g} \cdot I}{a^{2}}}
$$


where the constant terms influencing the photobleaching have been combined into the constant factor $C$. Keeping the correct sampling, $a$ is inverse proportional to the increase of resolution (between confocal and STED) $\rho=d_{\text {conf }} / d_{x, y \text { min }}=\sqrt{1+I / I_{s}}$, concrete $a=d_{\text {conf }} /(\rho \cdot \nu)$. Whereby $d_{\text {conf }}$ and $d_{x, y \text { min }}$ are the confocal and STED resolution (defined as the FWHM), respectively. $\nu$ is an oversampling factor also known as Nyquist criterion. Using these expressions, the equation 2.2 can be written as:

$$
S_{f l}=t_{i m g} \cdot \eta F_{0} \cdot e^{-C \cdot t_{i m g} \cdot \rho^{2}\left(\rho^{2}-1\right) \cdot \nu^{2}}
$$

The constant terms again were moved into $C$ for enhanced clarity. The maximum of equation 2.3 is at

$$
t_{i m g}=1 /\left(C \cdot\left(\rho^{4}-\rho^{2}\right) \cdot \nu^{2}\right)
$$

This best dwell time differs for each dye and resolution (level of illumination), because $C$ and $\rho$ is changing. The exponent of the exponential function is mainly influenced by the $\rho^{4}$ for high resolution enhancements. The maximum fluorescence signal $S_{f l, \max }$ which can be extracted is

$$
S_{f l, \max }=\frac{\eta F_{0}}{e \cdot C \cdot\left(\rho^{4}-\rho^{2}\right) \cdot \nu^{2}}
$$

The equation 2.5 shows a $1 / \rho^{4}$ dependency on the maximum fluorescence signal, since at higher resolutions more pixels have to be scanned with a higher STED illumination. Both, the STED intensity and pixel number, increase quadratically with the resolution enhancement. As a consequence, the signal drops rapidly when approaching very high resolutions. Please note that equation 2.5 does not take into account the bleaching by the excitation, that is why $S_{f l, \max }(\rho=0)=\infty$.

In order to optimize the signal, $\nu$ should be kept as low as possible above the Nyquist criterion, since it has a quadratic dependence on the bleaching light dose. In a typical scan (square pixels) the critical direction for the Nyquist frequency is the diagonal direction of the pattern. The diagonal extent of a pixel needs to be smaller than half the target resolution (FWHM) $d_{x, y \min } / 2$. Assuming a STED resolution of $30 \mathrm{~nm}$, the pixel size to fulfill the Nyquist criterion along the diagonal axis is $a<d_{x, y \min } /(2 \sqrt{2}) \approx 10.6 \mathrm{~nm}$.

As can be seen in equation 2.5, a doubling of the resolution results in an approximately 16-times lower maximum signal. Keeping $\rho$ and $\nu$ fixed, possibilities to increase $S_{f l, \max }$ are: a higher detection efficiency (increasing $\eta$ ), a higher labeling 


\section{Fluorescence Photon Budget and STED Resolution}

density and thus fluorescence level $F_{0}$, or a lowering of the constant parameters defining $C$.

One way to lower $C$ is to reduce the bleaching cross section of the specific dye. Another possibility is the promising approach to decrease the number of photons $\sum N_{p h}$ leading to photobleaching events. Reducing $\sum N_{p h}$ is the rationale of two different scan schemata presented in chapter 4 and 5 . For instance, a photobleaching reduction of a factor $\kappa$ will allow a higher $S_{f l, \max }$ by the same factor, if the dwell time is increased by $\kappa$. According to equation 2.5, the higher fluorescence signal can be converted into an approximately $\sqrt[4]{\kappa}$ higher resolution with the same fluorescence signal as before. As an example, a ten times higher maximum signal is convertible into a $\sim 45 \%$ higher resolution (i.e. $40 \mathrm{~nm} \Rightarrow 22 \mathrm{~nm}$ ), see figure 2.2 .

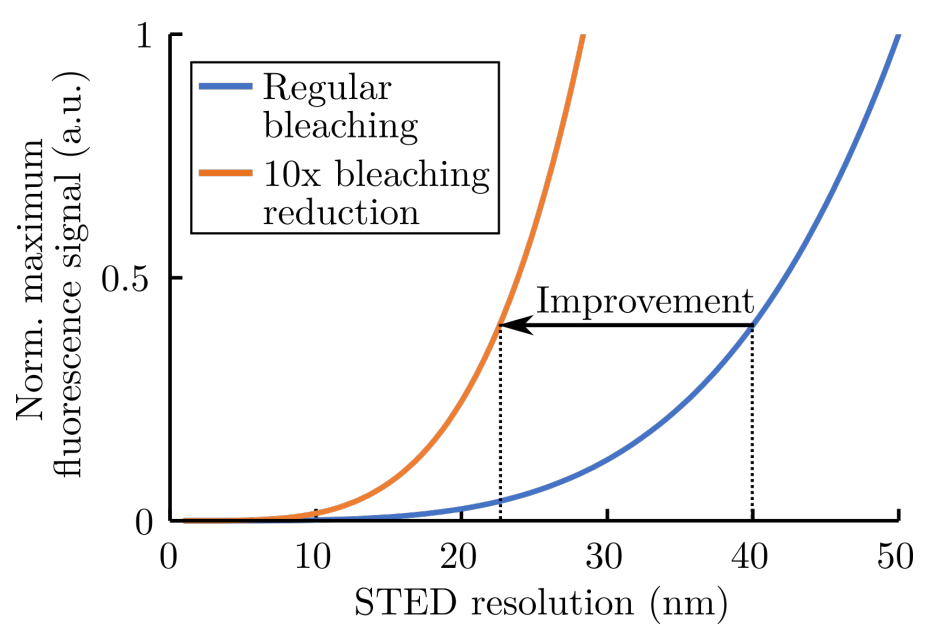

Figure 2.2.: Resolution improvement by a 10-fold bleaching reduction. Shown are plots of equation 2.5 for final resolutions below $50 \mathrm{~nm}$ with a different amount of photobleaching. The blue line represents the maximum signal for regular bleaching (e.g. $C=1$ ), and is nominated to a resolution of $50 \mathrm{~nm}$. The orange line shows the maximum signal with ten times lower bleaching (e.g. $C=1 / 10$ ) compared to the blue line. The relative resolution improvement between both is $\sim 45 \%$ for any starting resolution. 


\subsection{Collecting the maximum fluorescence signal}

In this section, equation 2.3 is proved. To do so, fluorescent nanoassemblies labeled with the dye ATTO 647N (Bead R, GattaQuant, see chapter 7) were imaged at two different resolutions and with different dwell times. For each resolution the pixel size was adapted according to the Nyquist criterion. In figure 2.3 the experimental data is shown, fitted with equation 2.3 As fitting variables only the constant factor $C$ and the initial fluorescence level $F_{0}$ were used ( $\eta$ should be constant). $C$ and $F_{0}$ were independently fitted (least squares) for both data sets and agree within $1 \%$ between both resolutions. The little difference between experiment and theory suggests that the here presented model incorporates most of the effects of the system.

It is highlighted that a better resolution and structure definition both require a high fluorescence signal. Keeping the resolution fixed, the fluorescence signal depends only on the fluorescence photon collecting efficiency $\eta$, the start fluorescence level $F_{0}$, the bleaching cross section $\sigma$, and the number of photons inducing bleaching events. With the model, the imaging settings can be optimized, and solutions to increase the fluorescence signal can be developed.

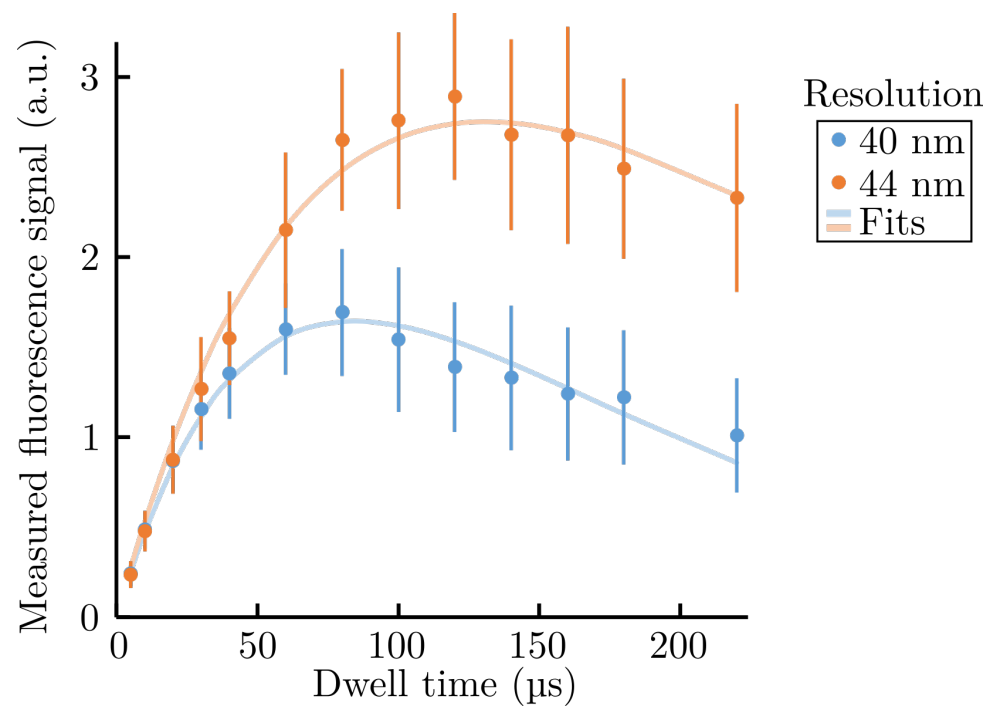

Figure 2.3.: The fluorescence signal in STED imaging in dependence on the resolution and dwell time. The obtained fluorescence signal as a function of the pixel dwell time for two different STED resolutions. $44 \mathrm{~nm}$ (orange) and $40 \mathrm{~nm}$ (blue), the pixel size was $17 \mathrm{~nm}$ and $15 \mathrm{~nm}$, respectively. Sample consisted of $\sim 23 \mathrm{~nm}$-sized fluorescent nanoassemblies (Bead R, GattaQuant). For each data point more than 200 different beads from 9 independent measurements were analyzed. Data is the mean value with \pm 1 st. dev. Fitting was performed with equation 2.3 . Figure reproduced from ref. [2]. 



\section{Illumination-Control using a Spatial Light Modulator}

As shown in section 2.2, the fluorescence signal is the main limiting factor for the maximum STED resolution. In this chapter, a spatial light modulator is used to create the phase masks for the OFF-switching light pattern. When focusing the phase-modulated wavefront, the OFF-switching pattern in the focal plane of the objective lens is created. The SLM is also used to correct aberrations and optimize the phase masks on the fly for those, leading to a higher fluorescence signal, and hence resolution.

\subsection{STED nanoscopy setup}

For this thesis a STED microscope with a spatial light modulator (LCOS-SLM; Hamamatsu Photonics) for manipulating the STED beam wavefront was developed, see figure 3.1. During the thesis two different STED lasers were used. In this chapter (3), a pulsed STED laser at $775 \mathrm{~nm}$ with a pulse width of $\sim 1.2 \mathrm{~ns}$ and a power of $1.25 \mathrm{~W}$ was used (MPB Communications, Canada). In chapter 2(Signal Optimum), chapter 4 (MINFIELD), and chapter 5 (DyMIN), a STED laser at $775 \mathrm{~nm}$ with a pulse width of $\sim 640 \mathrm{ps}$ and a power of $3.3 \mathrm{~W}$ was used. The latter was home-built from a seed laser working at $1550 \mathrm{~nm}$ with a power of $7.0 \mathrm{~W}$ (onefive, Switzerland).

The new optical implementation of the SLM allows to imprint two orthogonal and independent phase information into the same STED beam. A related approach, but with a different optical design, was reported earlier 93. Incoming light to the SLM is only phase-modulated if its polarization direction is parallel to the orientation of the liquid crystals inside the SLM. If the polarization of the light features an angle of $0^{\circ}<\theta<90^{\circ}$ in respect to the crystal orientation at the first encountering of the SLM, the beam is incoherently split into two beams, which are linear and orthogonal polarized. This effect is used by passing the SLM twice at a different spatial position, thereby rotating the polarization direction by $90^{\circ}$ in-between. Consequently, the STED light is split, and both parts are modulated by one of the both sides of the SLM depending on the polarization at each encounter. The intensity of the first 


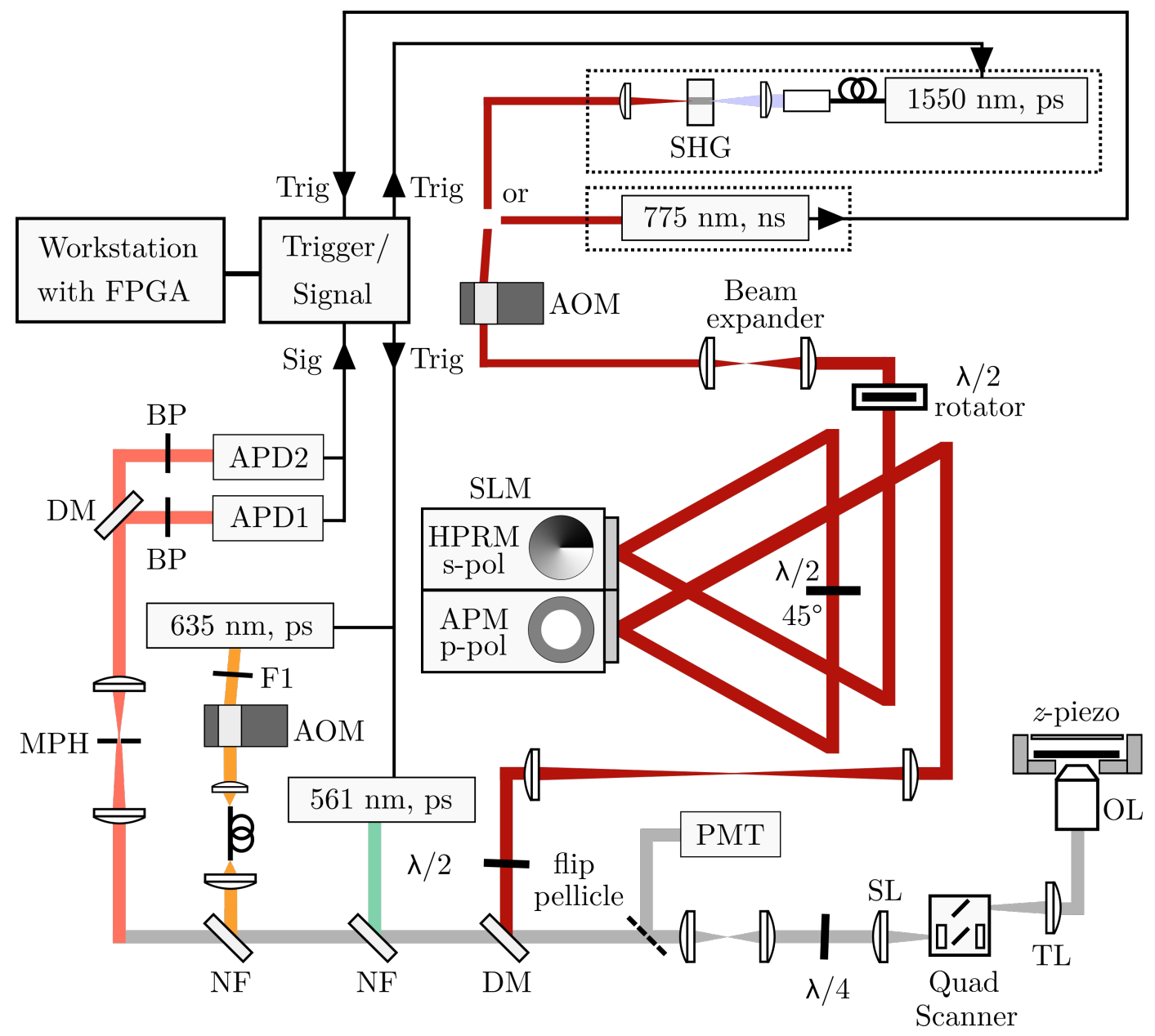

Figure 3.1.: Schematic representation of the relevant optical and mechanical microscope components. The STED beam phase is modulated by a spatial light modulator, which is imaged into the back aperture of the objective lens. By passing the SLM twice and in-between turning the polarization by $90^{\circ}$, the STED beam is incoherently split into two parts which are independently phase-modulated. SHG: second harmonic generation, ps: picoseconds, ns: nanoseconds, Trig: trigger, FPGA: field-programmable gate array, Sig: signal, AOM: acousto-optical modulator, BP: band pass, DM: dichroic mirror, APD: avalanche photodiode, SLM: spatial light modulator, HPRM: helical phase ramp mask, APM: annular phase mask, MPH: motorized pinhole, OL: objective lens, PMT: photo-multiplier tube, TL: tube lens, NF: notch filter, SL: scan lens.

beam part is $I_{1}=I_{0} \cdot \cos ^{2}(\theta)$, and for the second $I_{2}=I_{0} \cdot \sin ^{2}(\theta)$, with the initial intensity $I_{0}$. The intensity between both beams can be tuned with $\theta$ which is done by the $\lambda / 2$-rotator in front of the SLM. The used configuration for the phase masks in this thesis is a helical phase ramp mask (HPRM) for 2D-STED and an annular phase mask (APM) for axial-STED, see figure 3.1. Both resulting OFF-switching 
patterns are overlaid in the focus plane of the objective lens. By changing $\theta$, and therefore the power ratio between 2D- and axial-STED OFF-switching pattern, the resolution can be tuned in all three dimensions [57 [94.

The microscope features two excitation lasers with wavelengths of $635 \mathrm{~nm}$ and 561 $\mathrm{nm}$ (both Abberior Instruments), which were used for dual-color imaging with the same STED laser at $775 \mathrm{~nm}$. A similar approach was reported earlier 78, but with slightly different excitation wavelengths $(640 \mathrm{~nm}$ and $594 \mathrm{~nm})$. The excitation wavelengths used here, allowing a greater detection window for the fluorescence compared to the earlier approach, enabling a higher fluorescence photon budget and hence higher resolution.

For detection, avalanche photodiodes (APDs) were used with a detection window of $580-630 \mathrm{~nm}$ for the APD1 and $650-720 \mathrm{~nm}$ for the APD2 (both APDs: SPCMAQRH13; Excelitas, North America). The microscope worked at a repetition rate of $40 \mathrm{MHz}$, if not other stated.

The STED beam and both excitation beams (picosecond pulses) can be switched off and on within a few hundred nanoseconds. For the STED beam and $635 \mathrm{~nm}$ excitation beam, this is done by blocking the beams with an acousto-optical modulator (AOM) (AA Opto Electronic, France). The $561 \mathrm{~nm}$ excitation laser can be switched pulse-wise by modifying the trigger pulses. Scanning in the $x y$-plane was performed via galvanometric scanners (Cambridge Technology, United Kingdom) arranged in a quad scanner design 73 , and along the optical axis $z$ with a piezo stage (P-736.ZR2S; Physik Instrumente, Germany). The analog scan signals as well as the control signals to switch the laser beams either on or off were generated by a field programmable gate array (FPGA) 1 . A precise calibration of the scanner allows a reliable repositioning at any line frequency (especially at small scan fields as used for the MINFIELD approach). For the scanner calibration, the lag times at different scan frequencies were recorded and accordingly adjusted.

The optical setup was built around an Olympus IX83 microscope body, the objectives used were an UPLANSAPO 100XO (oil-immersion) and an UPLSAPO 60XW (water-immersion) (Olympus, Japan). The microscope was controlled by the software ImSpector v12 (Abberior Instruments, Germany).

${ }^{1}$ The software for scanning was developed by Dr. Andreas Schönle. 


\section{Illumination-Control using a Spatial Light Modulator}

\subsection{OFF-switching pattern enhancement}

The OFF-switching pattern plays a major role in coordinate-targeted superresolution microscopy. Here, the shape and quality of the OFF-switching minimum are optimized by the use of the SLM. Therefore not only the basic phase masks are created, but also corrections for optical aberrations are applied. With the optimized patterns for 2D-STED and axial-STED, a significantly higher fluorescence signal is achieved.

\subsubsection{D-STED}

One common aberration in an imaging system is astigmatism, whose influence on the 2D-STED OFF-switching pattern and signal is shown in figure 3.2 By scanning reflecting gold nanoparticles with a diameter of $150 \mathrm{~nm}$, the STED OFFswitching pattern shape is investigated. In STED microscopy, astigmatism leads to an elliptically shaped doughnut, which is shown in figure $3.2(\mathrm{~A}+\mathrm{C})$. In comparison, figure 3.2 (B) shows an astigmatism-corrected doughnut with an almost perfectly round shape. Some astigmatism corrections are applied to achieve the shape in (B). Taking this point as a nominal reference, the fluorescence signal was measured as a function of astigmatism using fluorophore nanoassemblies (Beads R, GATTAquant, Germany). Those beads have a very low variance in brightness, and are hence a sensitive sample to detect small changes in the fluorescence signal due to alteration of the OFF-switching pattern minimum. In figure 3.2 (D) the measured and normalized fluorescence signal (black dots) in dependence on the Zernike polynomial of astigmatism is shown. The STED power in the sample was $115 \mathrm{~mW}$. The drop in brightness is directly connected to the induced astigmatism onto the 2D-STED OFFswitching pattern, as theoretically proposed by Otomo et al. 95. Please note that other third-order aberrations were also slightly corrected before the measurements of figure 3.2 were performed.

The experimental data is in good agreement with the simulation ${ }^{2}$ (dotted line), see figure 3.2 (D). The small differences between both can be caused by small amounts of STED light in the OFF-switching pattern minimum center even without astigmatism. This would reduce the fluorescence signal (in contrast to the simulation), which flattens out the curve around zero astigmatism due to the nonlinear square root dependence of the STED effect. In the experiments, a moderate astigmatism of \pm 0.15 already led to a drop of the fluorescence signal of roughly $20 \%$ at the applied STED power of $\sim 115 \mathrm{~mW}$. Of course at higher STED powers, the relative impact of astigmatism on the fluorescence signal would be even higher.

\footnotetext{
${ }^{2}$ Simulation were performed by Dr. Jan Keller-Findeisen and are included for clarity.
} 

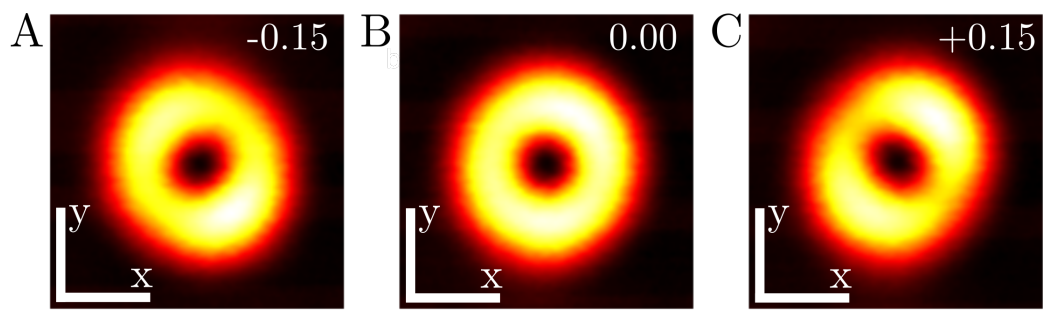

$\mathrm{D}$

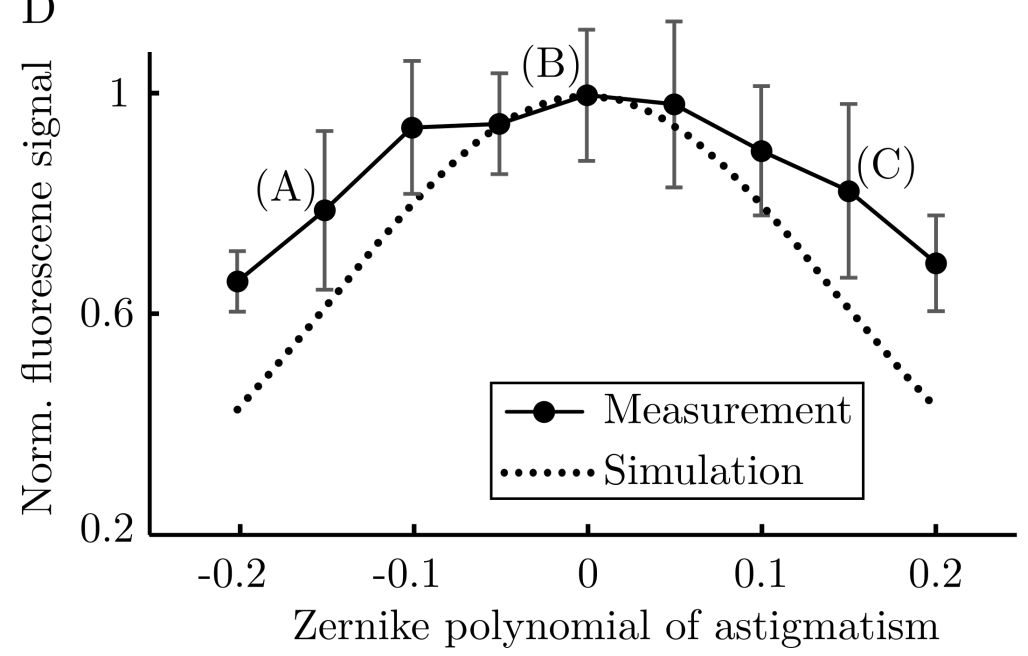

Figure 3.2.: Influence of astigmatism on the 2D-STED OFF-switching pattern and fluorescence signal. (A) astigmatism of $-0.15,(\mathbf{B})$ corrected astigmatism and $(\mathbf{C})$ astigmatism of +0.15 . (D) Measured (dots) and simulated (dotted line) influence of astigmatism on the attainable fluorescence signal. Black continuous line is a guide to the eyes. Negative and positive astigmatism both lead to a (symmetric) drop in the 2D-STED fluorescence signal. Sample consisted of $\sim 23 \mathrm{~nm}$-sized fluorescent nanoassemblies (Bead R, GATTAquant). Scale bars $(x, y)$ are $200 \mathrm{~nm}(\mathrm{~A}, \mathrm{~B}, \mathrm{C})$.

This highlights the need for aberration-corrected imaging systems to get the best 2D-STED performance and especially the highest fluorescence signal and resolution. 


\section{Illumination-Control using a Spatial Light Modulator}

\subsubsection{Axial-STED}

To create the OFF-switching pattern for axial STED, a few conditions need to be matched. Theoretically, with a plane wave used, the diameter $d_{\text {circle }}$ of the phase mask inner circle ( $\pi$-delay) is $\sim 0.71 \cdot d_{B A}[96$, with the back aperture diameter of the used objective lens $d_{B A}$. The size of the back aperture can be calculated as

$$
d_{B A}=2 \cdot \frac{M_{o b j}}{f_{T L}} \cdot N A
$$

with the magnification of the objective lens $M_{o b j}$ and the focal length of the tube lens $f_{T L}$ (e.g. $180 \mathrm{~mm}$ for the Olympus body used in this thesis) 97]. This matching condition is important if the objective lens is changed, since the back aperture size varies with a different magnification and NA. In contrast to the theoretical diameter, slightly lower circle diameters are used because the wavefront is not a perfectly plane wave but approximately the inner part of a Gaussian intensity distribution. However, with the SLM as the phase mask element, the optimum diameter is easily adjustable and can also be adapted to different objective lenses.

The optimum circle diameter was first experimentally found by optimizing the fluorescence signal while continuously imaging fluorescent nanobeads (Crimson beads, nominally $40 \mathrm{~nm}$ diameter). The circle diameter giving the highest signal is defined as the nominal diameter (1.0). All other diameters refer to this one. In figure 3.3 the influence of the phase mask circle diameter on the OFF-switching pattern shape is displayed. To measure the OFF-switching pattern intensity distribution, the patterns were scanned above reflecting gold nanoparticles with a nominal diameter of $150 \mathrm{~nm}$. The reflected signal was detected with a photo-multiplier tube.

A wrongly adjusted circle diameter of 0.8 results in an OFF-switching pattern which features no deep minimum (Figure 3.3 (A, top). The line profile (bottom) represents the intensity distribution orthogonal to the optical axis in the nominal focus plane. Clearly, the condition for destructive interference in the focus point is not met. With this kind of OFF-switching pattern, no useful STED image is possible, since the fluorescence is switched OFF everywhere. In contrast, the optimized axial-STED OFF-switching pattern with a phase mask circle diameter of 1.0 (Figure 3.3 (B, top)) has a deep central intensity minimum as highlighted by the associated line profile (B, bottom). With this deep minimum, a good fluorescence signal can be expected, as well as a good resolution for a certain STED power, because the slope of the OFF-switching edges along the $z$-direction and around the minimum should be close to the diffraction limited maximum. A relative circle diameter of 1.2 also results in a distortion of the axial-STED OFF-switching pattern, whereby the line profile resembles only one maximum, figure 3.3 (C, bottom). 

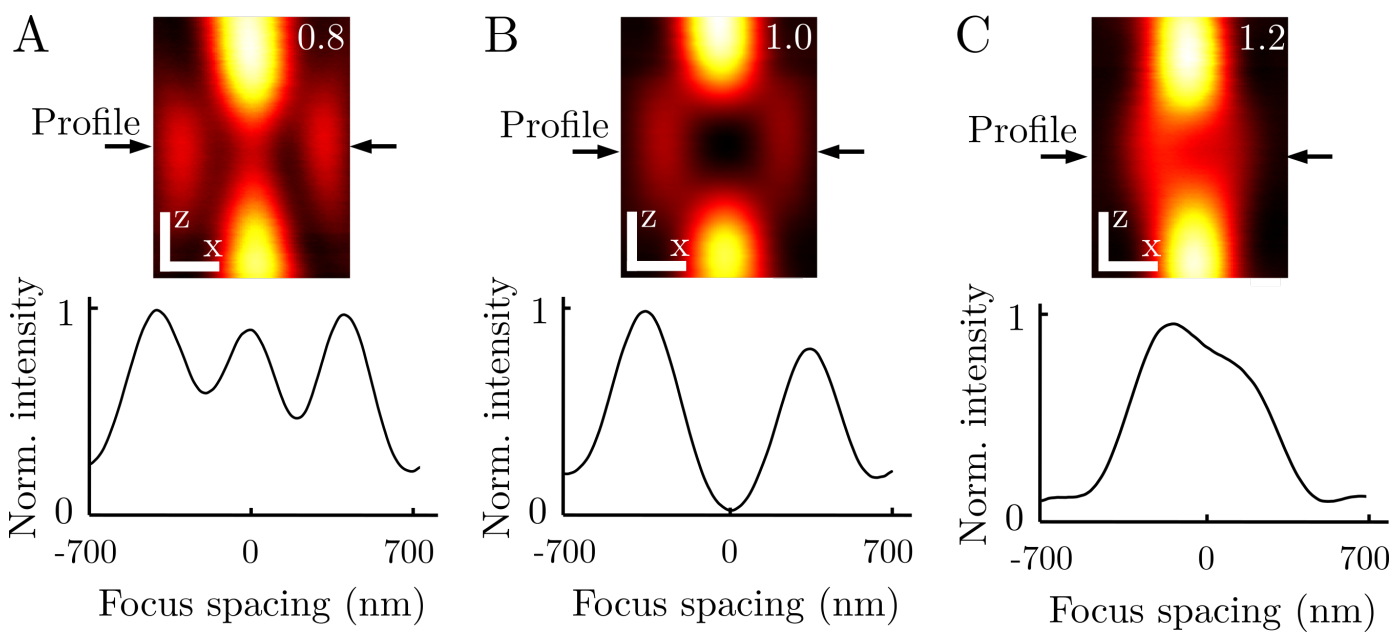

Figure 3.3.: Measured axial-STED OFF-switching pattern shapes in dependence on the phase mask circle diameter. Sample: gold nanoparticles. (A) Axial-STED OFF-switching pattern resulting from a nominal phase mask circle diameter of 0.8 (top). Line profile (bottom), no central minimum is present. (B) Axial-STED OFF-switching pattern (top) with the nominal phase mask circle diameter of 1.0 (optimum). The line profile (bottom) shows a deep minimum. (C) Axial-STED OFF-switching pattern (top) corresponding to a nominal phase mask circle diameter of 1.2. The line profile (bottom) does not feature a minimum. Scale bars $(x, z)$ are $200 \mathrm{~nm}(\mathrm{~A}, \mathrm{~B}, \mathrm{C})$.

Figure 3.4 shows the normalized fluorescence signal as a function of the relative circle diameter of the axial-STED phase mask. The measured data points (black dots) represent the fluorescence signal which decreases fast, if the circle diameter of the phase mask is not well set.

The simulation of the experiment ${ }^{3}$ (Figure 3.4 , dotted line) agrees well with the measured data (Figure 3.4 , black dots). In both, simulation and experiment, a circle diameter mismatch of $5 \%$ results in a drop of the fluorescence signal of more than $60 \%$ at the used STED power of $230 \mathrm{~mW}$. The drop of the fluorescence signal is connected to the STED light which occurs in the minimum for the mismatched case. This amount of STED light switches OFF the fluorophores following the square root dependence on the STED intensity. Already a little STED light leads to a relatively large loss of fluorescence, which also scales with the overall applied STED power. At higher resolutions (i.e. STED powers) than used here, an even greater relative fluorescence signal drop will be present for the same circle diameter mismatch.

A very large circle diameter mismatch can occur when switching between different objective lenses (e.g. from an oil-immersion to a water-immersion objective). To

\footnotetext{
${ }^{3}$ Simulation were performed by Dr. Jan Keller-Findeisen and are included for clarity.
} 


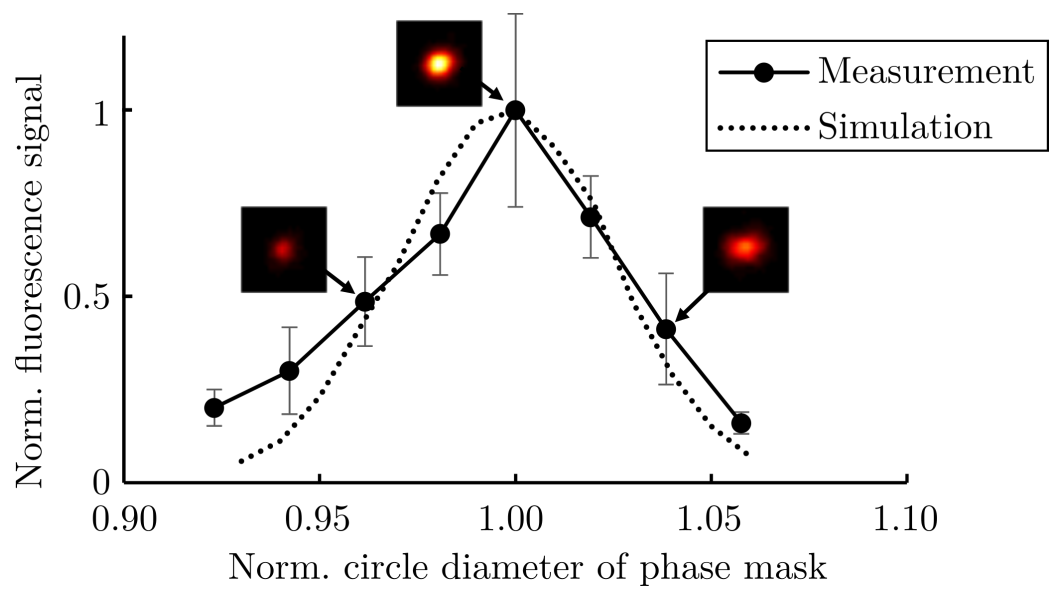

Figure 3.4.: Dependence of the fluorescence signal on the phase mask circle diameter. The measured normalized fluorescence signal (black dots) of dispersed $40 \mathrm{~nm}$-sized Crimson beads vs. the normalized phase mask circle diameter. The black continuous line is a guide to the eyes. A relative mismatch of $4 \%$ results in a fluorescence signal drop of $\sim 60 \%$ at this STED power of $\sim 230 \mathrm{~mW}$, as also found by a simulation (dotted line). Insets are representative of the data set, identical look-up table are used (normalized to the brightness according to a circle diameter of 1.0).

achieve a good axial-STED OFF-switching pattern for any objective, the circle diameter needs to be readjusted. With the SLM-based phase mask design this requirement is given, featuring a wide biological application range.

\subsection{Axial-STED in live-cell embedded samples}

Beside the correct phase mask circle diameter, it is important for a good axial-STED imaging result that no aberrations distort the OFF-switching pattern. In living cells a distortion happens by the rise of spherical aberration when imaging inside of the sample. This aberration occurs, because of the difference of the refraction index between the oil-immersion of the objective lens, and sample embedding medium. The achievable resolution of the system is worse, because the fluorescence signal is suppressed.

For live-cell embedding, a water-based solution is typically used with a refractive index of $n_{e m b} \approx 1.33$. Living tissue or organisms have a little higher index. The refractive index of an oil-immersion objective is typically $n_{\text {oil }}=1.518$. The large difference results in a quick rise of spherical aberrations linearly with the penetration depth [56] 98.

Close to the coverslip surface, the spherical aberration is normally zero. Depending on the refraction index mismatch, the used STED power, and the penetration depth, 
the distortion of the OFF-switching minimum becomes so severe that imaging with axial-STED is hardly possible. In earlier works, spherical aberrations where compensated to a certain point using an SLM only in the STED beam path [58, or for all beams (illumination, STED and detection) using a deformable mirror 99 . However, both implementations demonstrated corrections only for fixed samples which have an embedding medium with a higher refraction index compared to water. The much higher spherical aberrations in living samples should be correctable in a comparable fashion, but only to smaller depth, keeping the good resolution of the high NA oil-immersion objective lens (see section 3.3.2). In any case, imaging at great depth requires a better matching of the refraction indexes of immersion liquid and embedding medium. This is easily viable with a water-immersion objective, but at the cost of a lower resolution due to the lower NA.

\subsubsection{Imaging depths for different objectives}

The axial-STED performance with an oil-immersion and a water-immersion objective is shown in figure 3.5 for a water-embedded sample. The technical sample consisted of two quasi mono-layers of fluorescent nanobeads (40 nm, Crimson beads, Molecular Probes) which were spaced $10 \mu \mathrm{m}$ apart from each other (Figure 3.5(A)). With an oil-immersion objective (Figure 3.5 (B)), hardly any signal is observed for the confocal and axial-STED recording. The spherical aberrations at this depth lead to strong blurring of the confocal excitation and STED focus intensity distributions. Also an axial shift in respect to the nominal position of the inner layer is observed as spherical aberrations are linked to defocus [98. In comparison, the inner layer is well recorded when using a water-immersion objective (Figure 3.5 (C)). Alternatively, the inner layer of the sample was placed on the concave part of a well objective slide (Figure 3.5 (D)) to allow a continuous variation of the distance between both layers (sample scheme and more data shown in appendix A.4. Up to a maximum depth of $180 \mu \mathrm{m}$, which was limited by the objective lens working distance, the same image quality and resolution was observed. With the use of a water-immersion objective, axial-STED can reach in principle a high imaging depth, which is partly necessary for live-cell or tissue investigations. 


\section{Illumination-Control using a Spatial Light Modulator}
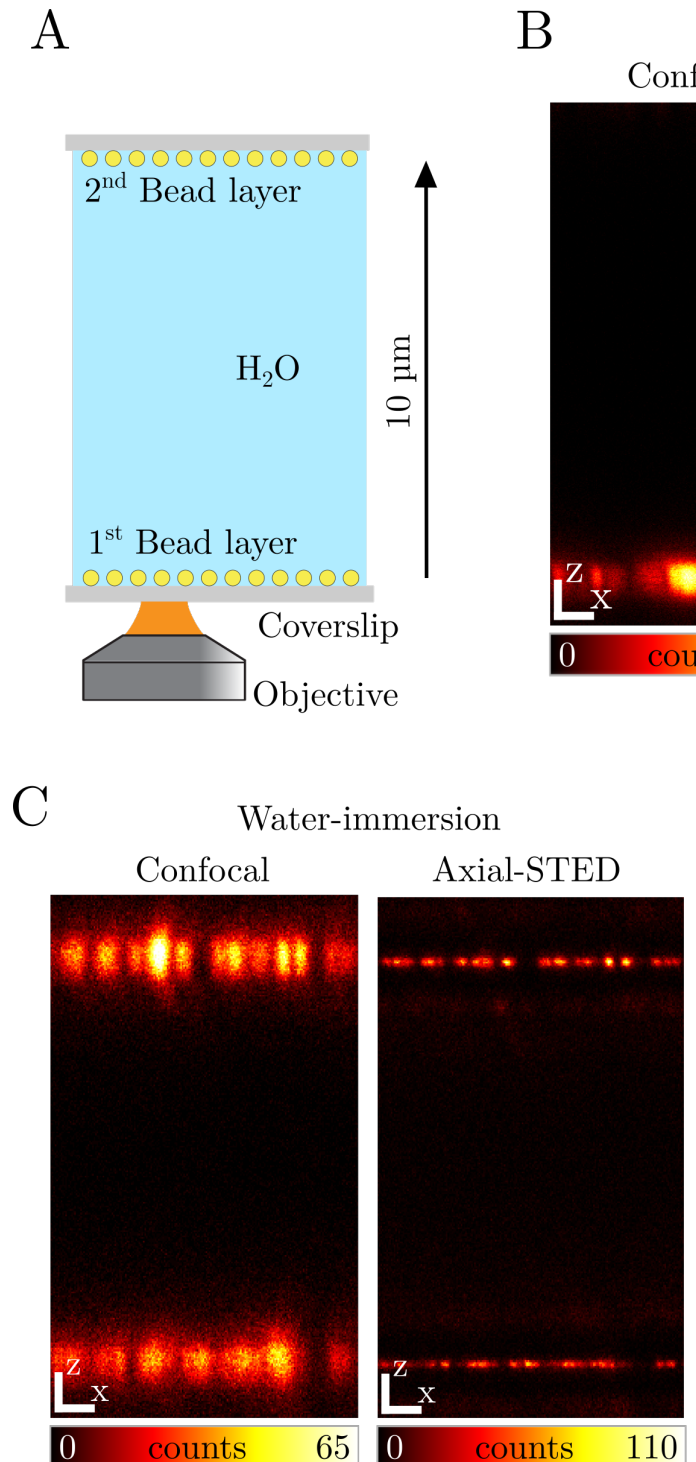

B Oil-immersion

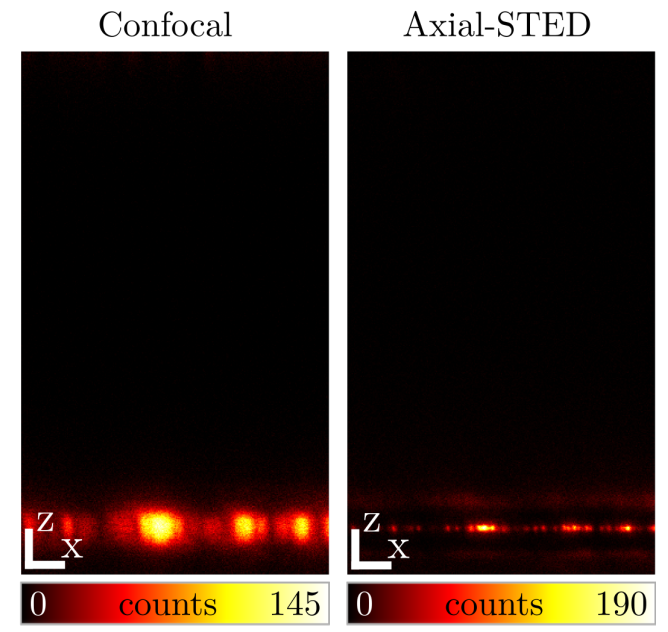

D Water-immersion
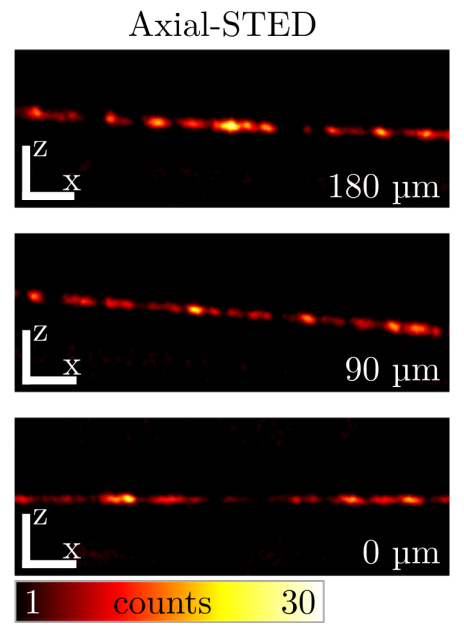

Figure 3.5.: Objective lens dependent penetration depths in waterembedded samples with axial-STED. (A) Scheme of the sample. Two quasi mono-layers of $\sim 40 \mathrm{~nm}$-sized fluorescent nanobeads (Crimson, Molecular Probes) spaced $10 \mu \mathrm{m}$ apart are embedded in water. (B) Confocal (left), and axial-STED (right) recordings, with an oil-immersion objective. The inner bead layer is hardly visible in the confocal image, and not visible in the STED image. (C) Here, a water-immersion objective was used for acquisition, confocal (left) and axial-STED (right). In contrast to (B), both bead layers are imaged with the same fluorescence signal and resolution. (D) Sample: the inner bead layer (top, middle) is placed on a well slide, allowing a variation of the distance between the inner and outer (bottom) layer, up to the maximum depth of $180 \mu \mathrm{m}$. The individual bead clusters are resolved without any signal loss. Scale bars $(x, z)$ are $1 \mu \mathrm{m}$ for $(\mathrm{B}, \mathrm{C}, \mathrm{D})$. 


\subsubsection{Correction for spherical aberrations}

An oil-immersion objective is used to image a water-embedded fixed sample, leading to strong spherical aberrations. Figure 3.6 shows $x z$-measurements for which nuclear pore complex 153 was labeled with STAR RED via indirect immunofluorescence (Abberior, Germany). Figure 3.6 (A) shows a confocal image of the structure without significant signal loss up to the maximum structure depth of $\sim 3 \mu \mathrm{m}$.

However, using axial-STED without correcting spherical aberrations (Figure 3.6 (B)) leads to a fast decrease in the fluorescence signal when imaging inside the sample. The OFF-switching pattern minimum distortion around a depth of 1 $\mu \mathrm{m}$ forbids collecting enough signal for common STED powers in the range of $50-200 \mathrm{~mW}$. Only the nuclear pores close to the coverslip are bright enough for a clear structure representation. When using the SLM to correct for spherical aberration, the maximum penetration depth was enhanced to $3 \mu \mathrm{m}$ (Figure 3.6 (C)). Now, in contrast to (B), the nuclear pores on the inside are imaged with the same fluorescence signal.

The maximum depth at which a correction is still possible depends on the steepest phase gradient the SLM can provide within two pixels $(0-2 \pi)$. Note that pushing the maximum penetration depth to $\sim 5 \mu \mathrm{m}$ should also be possible but depends on the applied STED power and the area and hence pixels used on the SLM altering the wavefront.

During the measurement, the different degrees of spherical aberration were not continuously corrected with the penetration depth on the SLM, but in discrete (in this case five) steps. The binning of the corrections avoids a permanent chance of all SLM pixel values (which have a rise and fall time of a few ten milliseconds), leading to a better stability of the OFF-switching pattern. 
3. Illumination-Control using a Spatial Light Modulator
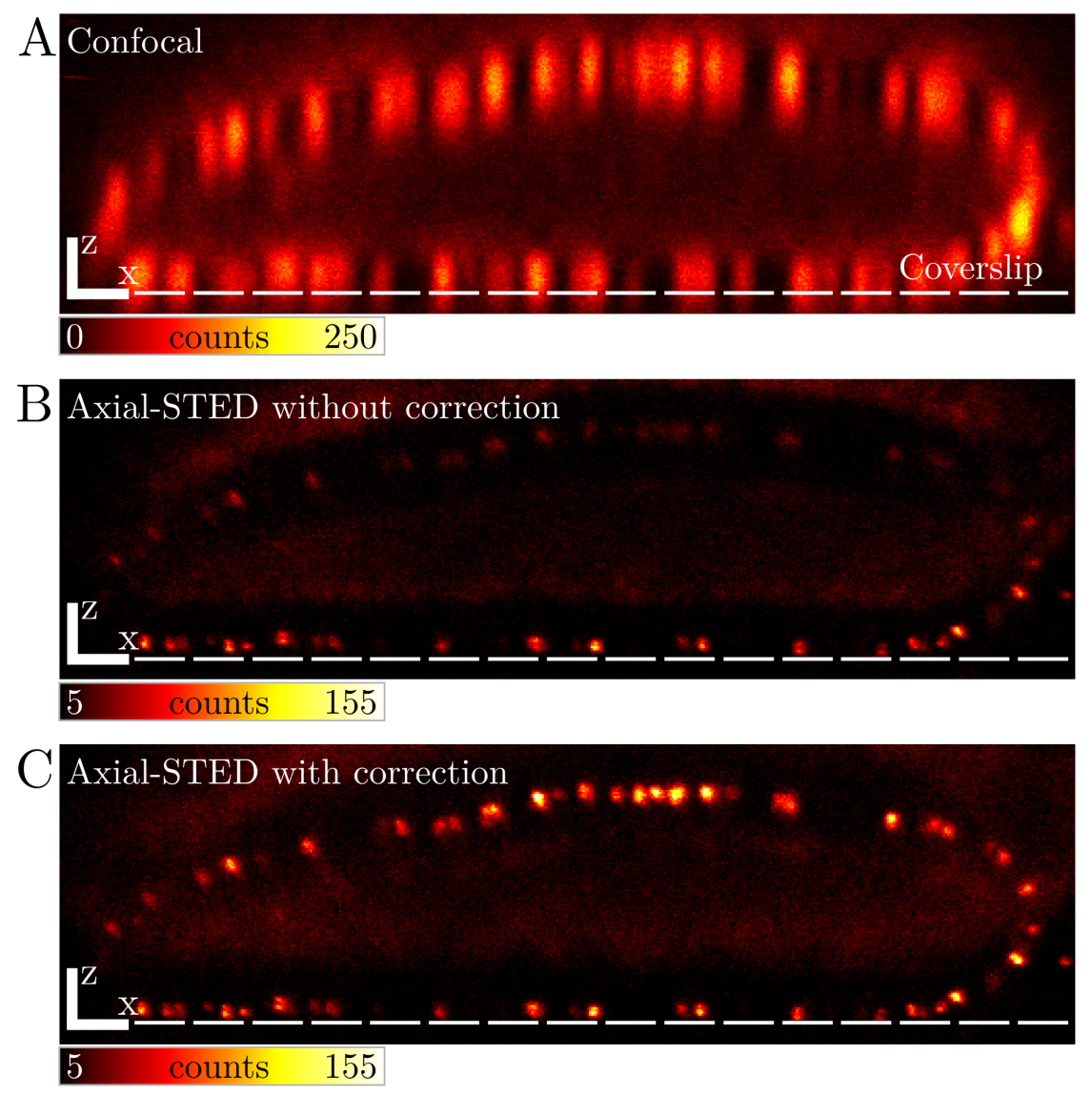

Figure 3.6.: Correction of spherical aberrations increases the penetration depth with axial-STED. Sample: nuclear pore complexes, indirect immunofluorescence labeling against Nup153 (STAR RED, Abberior). The cell is mounted in water and imaged with an oil-immersion objective. (A) Confocal recording. (B) Axial-STED without spherical aberration correction is only possible up to a depth of $\sim 0.5-1.0$ textmu $\mathrm{m}$ at this STED power (resolution). (C) Same recording as (B) but with spherical aberration corrected via the SLM. All the NPCs up to a depth of $\sim 3 \mu \mathrm{m}$ are imaged with the same fluorescence signal. Scale bars $(x, z)$ are $1 \mu \mathrm{m}$ $(\mathrm{A}, \mathrm{B}, \mathrm{C})$. 


\subsubsection{Live-cell imaging}

In a live-cell sample tubulin was stained with silicone rhodamine ( $\mathrm{SiR}$ ) (see Materials and Methods). The sample was imaged with a water-immersion objective, . Figure 3.7 depicts an $x z$-measurement in confocal (Figure 3.7 (A)) and axial-STED mode (Figure 3.7 (B)) of two cells being $37 \mu \mathrm{m}$ apart along the optical axis. All tubulin strands perpendicular to the imaging plane appear as distinct, highly resolved round spots, regardless of the depth they are situated in. This is also highlighted by figure 3.7 (C) which illustrates the line profile between the two white arrows in figure 3.7 (B). Three tubulin strands are very well separated by axial-STED (orange line) as compared to confocal imaging (blue line). The fitted data (gray line) agrees excellently with the experimental results. An axial resolution of $\sim 153 \mathrm{~nm}$ was achieved at this high penetration depth.
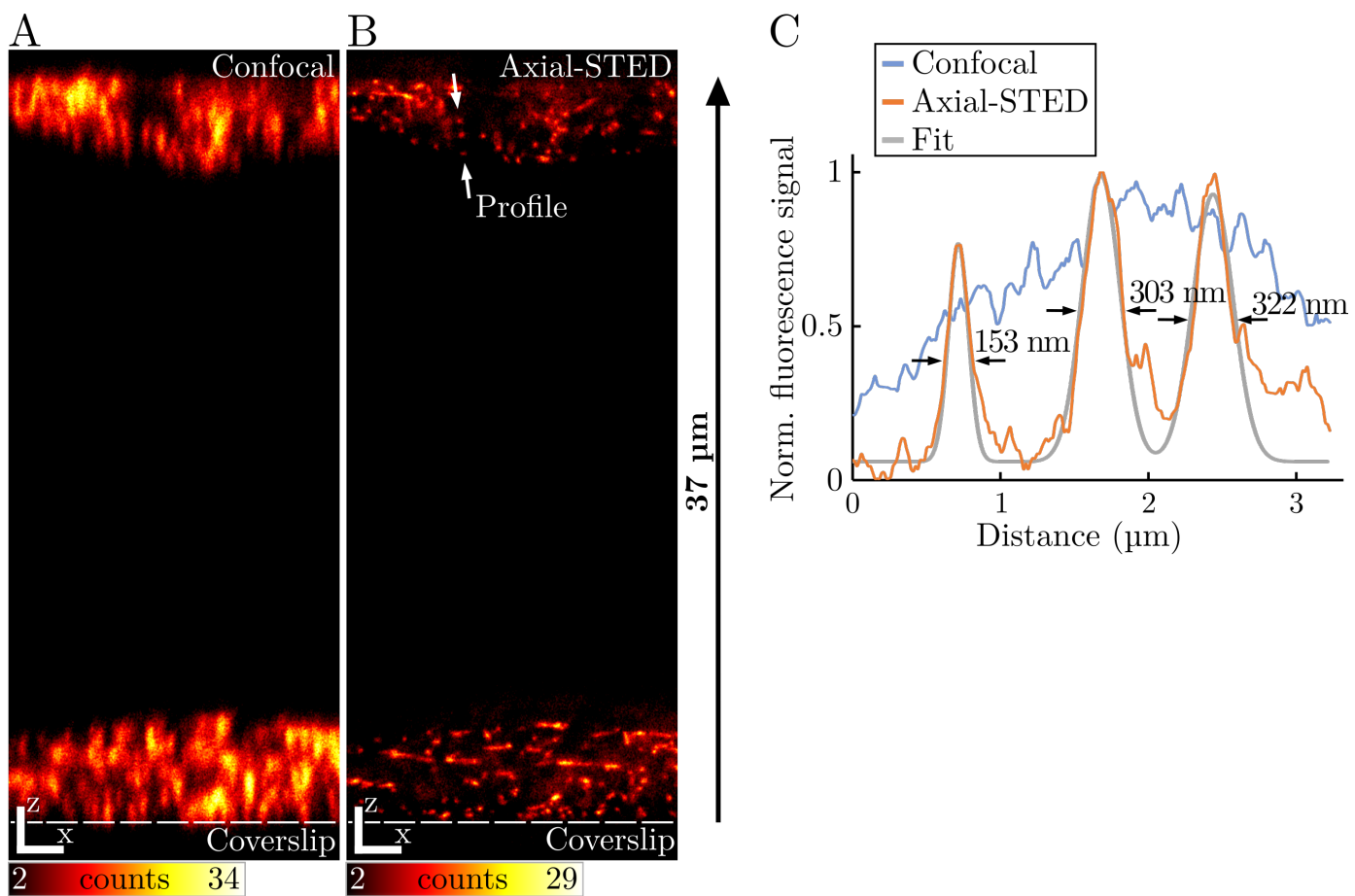

Figure 3.7.: Imaging of living cells with axial-STED at great depth. Sample: two living fibroblasts (stained with SiR tubulin) are arranged on opposite coverslips with a separation of $37 \mu \mathrm{m}$. Data ( $x z$-plane) were recorded using a waterimmersion objective. (A) Confocal image. (B) The tubulin is imaged with a good axial-STED resolution and at the same image quality at any depth. (C) Confocal (blue line) and axial-STED data (orange line) line profile which is indicated by the two white arrows in (B). A triple-peak Gaussian fit (gray line) of the data indicates a resolution of $\sim 153 \mathrm{~nm}$ at $\sim 32 \mu \mathrm{m}$. Scale bars $(x, z)$ are $2.5 \mu \mathrm{m}(\mathrm{A}, \mathrm{B})$. 



\section{Simpler MINFIELD Illumination}

In this chapter, the recently published MINFIELD scanning [1 is realized with galvanometric scanners for beam steering. The concept is also extended to 3DSTED and experiments have been performed in the greenish dye spectrum.

\subsection{Introduction}

As described, photobleaching is the limiting factor for STED microscopy. To achieve the required intensity $I_{s}$ around the doughnut minimum for ON/OFF-separation, the maximum intensity at the doughnut crest has to be several times higher due to the diffraction-limited size of the doughnut PSF. In a regular scan, these high intensities are scanned across the sample without contributing to the STED resolution, but induce photobleaching of the marker molecules [1] [89]. In the MINFIELD concept those excess intensities of the doughnut are sidestepped to a single small subdiffraction-sized scan area (located in the OFF-switching minimum center), and therefore do not contribute to the bleaching within the scan field. Typically, this field has a size of $50-200 \mathrm{~nm}$ in the lateral plane. To make use of the bleaching reduction, the scan area is repetitive imaged until the fluorescence level is decreased to typically $1 / e$. For the final image, the fluorescence signal of all images is summed up. This scan scheme avoids to a large extent a spatially non-linear bleaching behavior in the scan area. If the fluorescence level would have been bleached to $1 / e$ in only a single scan, the first rows of the image would be brighter, the last rows much dimmer.

The bleaching reduction by MINFIELD strongly depends on the size of the scanned field. Within a smaller field the bleaching is lower. Two different effects lower the bleaching light dose. At moderate scan field sizes $(>150 \mathrm{~nm})$, a higher fraction of the excess OFF-switching light is guided into the actual scan field (while imaging the outer pixels). However, this amount decreases non-linearly for smaller fields because the OFF-switching pattern has a quadratic intensity course around the minimum [47. Thus at small fields $(<150 \mathrm{~nm})$, the reduction of the OFF-switching light becomes more and more effective for smaller scan fields. This is the rational behind MINFIELD, and the first effect. But even if this non-linear benefit of the doughnut shape does not hold true for intermediate scan field sizes, still a great bleaching reduction can be achieved. Throughout a regular scan, each pixel is 


\section{Simpler MINFIELD Illumination}

illuminated with each part of the doughnut. The light dose $D$ a pixel receives is the integral over the doughnut intensity pattern times the pixel dwell time $t_{i m g}$. For intermediate field sizes, not the full doughnut intensity is integrated, but only a small fraction of it. This leads to a significant illumination reduction, which is the second effect. It can be thought of as the geometric light dose reduction (the scanned area and hence pixel number is less) when scanning smaller sub-diffraction scan fields. 2D-STED MINFIELD is very effective and can increase the fluorescence signal up to $\sim 100$-fold compared to a conventional scan [1].

In the first MINFIELD implementation, very fast electrooptical scanners were used to move the focused beams in the sample. There were two reasons why this type of scanner was used. First, those scanners feature a precise re-positioning which is necessary for imaging with few nanometers of resolution. Second, the scanners can move the focused beams so fast in the focal plane that the illumination can be continuously on while acquiring several images of the same area. If the scanners were too slow, a continuous illumination during the return motion of the scanner would result in unwanted illumination and photobleaching.

However, the optical microscope design with electrooptical scanners is more complex compared to the design with galvanometric scanners, limiting the possibility for additional optics. Furthermore, with electrooptical scanners only a small over all scan area (roughly $10 \times 10 \mathrm{~m}^{2}$ ) is accessible. Both drawbacks limit the imaging capabilities of the microscope for conventional scanning. A microscope design to perform high-quality MINFIELD and conventional scanning is desirable to facilitate a wide range of biological and technical applications.

\subsection{MINFIELD with galvanometric scanners}

In this thesis, galvanometric scanners are used to realize MINFIELD imaging with a conventional scanner setup. Those scanners move the beam in a classic manner by rotating a mirror around an axis, accelerated by a magnetic field. Due to the mass of the mirror and actuator, and the finite acceleration, the movement of the beam cannot be infinitely fast. When a line of the field is scanned, the scanner needs some time for the flyback (return motion), as seen in figure 4.1. During this time, the excitation and the STED beams are blocked by the AOMs (see section 3.1).

Only during the linear range of the mirror movement, both beams are guided to the sample. However, the beam on/off-modification has a certain rise and fall time, resulting in a little unwanted illumination in each scanned line. Let us assume the following scan parameters: pixelsize $a=5 \mathrm{~nm}$, MINFIELD size $\Delta s=150 \mathrm{~nm}$, a dwell time of $t_{i m g}=10 \mu \mathrm{s}$, and a rise/fall time of $t_{\text {rise }}=0.5 \mu \mathrm{s}$. The relative light dose overhead is $2 \cdot t_{\text {rise }} /\left(\frac{\Delta s}{a} \cdot t_{i m g}\right) \cdot 100 \% \approx 0.35 \%$, a negligible amount. 


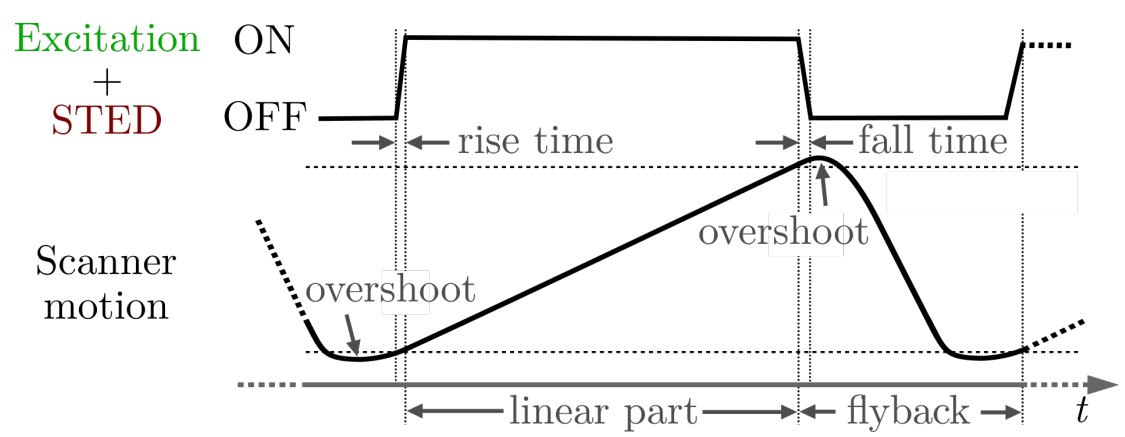

Figure 4.1.: Galvanometric beam scanner motion and laser control logic for a certain scan range. During the linear motion part, the individual image line is acquired, while the excitation and STED laser are guided to the sample. During the finite flyback time, both lasers are blocked to avoid unnecessary sample illumination.

Without switching the lasers off, the light dose during the flyback would be $\sim 280 \%$ higher compared to the dose which is necessary to image a specific line for this set of parameters. The scan concept with galvanometric scanners and AOMs has no disadvantage in terms of the illumination dose.

\subsection{D-STED MINFIELD}

In this section, the scan concept with galvanometric scanners for MINFIELD is applied for 2D-STED. It is important to test if the structure is blurred by the jitter of the scanners. MINFIELD measurements with different field sizes for three different known biological structures are shown in figure 4.2. A confocal image (A) of fluorescent nanobeads (Crimson beads, Molecular Probes), illustrates the much lower resolution compared to the 2D-STED MINFIELD images (B-D). In a typical MINFIELD scan, the excitation and OFF-switching PSFs are larger compared to the scanned area.

Images of the peripheral transmembrane protein gp210 of an amphibian cell are shown in figure 4.2 (B). This structure was reported earlier $[78$ and is known to form an 8-fold symmetry with an diameter of $\sim 160 \mathrm{~nm}$. Here, the structure is resolved by MINFIELD with a high resolution and excellent signal-to-noise ratio. With the used $200 \mathrm{~nm}$-sized MINFIELD, a 5 -fold signal gain is expected [1. It is important to note, that the galvanometric scanning features a sufficient repeatability between each scanned line and frame (ten frames summed up for images in (B)). No distortion along the fast $(x)$ or along the slow axis $(y)$ is observed.

Individual immature HIV-1 virions are shown in figure 4.2 (C). Direct labeling was performed against Gag.CLIP with silicon rhodamine (SiR-CLIP) following the 


\section{Simpler MINFIELD Illumination}

protocol in 100. Gag (group-specific antigen) proteins are necessary for assembling virus-like particles [101]. Due to the direct labeling, the marker molecule is closer to the labeled structure and therefore the structure is not blurred by the anitbody tree (indirect immunolabeling can add $>200 \mathrm{~nm}$ to the structure). This becomes a more important factor at resolutions below $30 \mathrm{~nm}$, e.g. achieved with MINFIELD [1]. Figure 4.2 (D) represents clathrin clusters in fixed Vero cells. With a MINFIELD size of $200 \mathrm{~nm}$, different morphologies 22 are clearly visible. The clathrin protein is important for the formation of coated vesicles 102 .

The use of galvanometric scanners for MINFIELD shows no disadvantage compared to electrooptical scanners but the simpler microscope design features a bigger application range besides MINFIELD imaging.
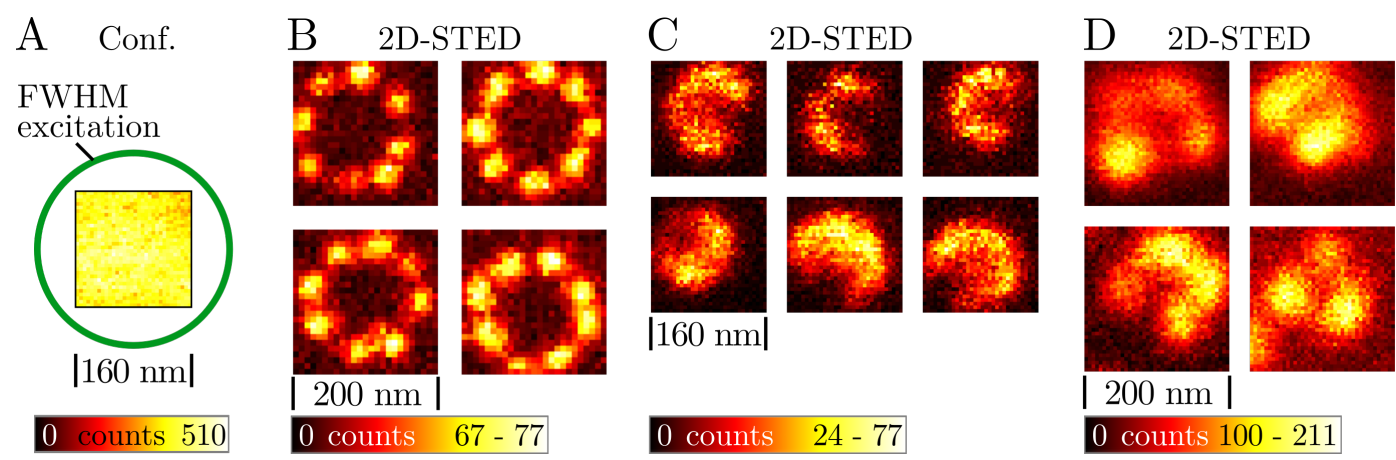

Figure 4.2.: 2D-STED MINFIELD with galvanometric scanners. (A) Confocal image with a MINFIELD-sized scan area of fluorescent nanobeads (Crimson beads, Molecular Probes). The excitation spot (and doughnut) size exceeds the scan area by far. (B) Amphibian nuclear pore complexes (NPCs) (indirect immunofluorescence, anti-gp210, STAR 635p) resolved by STED. The size of the MINFIELD is $200 \mathrm{~nm}$. (C) Immature HIV-1 virions (direct Gag.CLIP-labeling with silicon rhodamine (SiR-CLIP)). Size of the MINFIELD is $160 \mathrm{~nm}$, the structure of the virions is well resolved. (D) Nanoscale morphology of clathrin clusters in Vero cells (indirect immunofluorescence, STAR 635p). The size of the MINFIELD is $200 \mathrm{~nm}$. For each measurement, a lookup table from zero counts to the individual maximum is applied. 


\subsection{D-STED MINFIELD}

In addition to 2D-STED MINFIELD, a similar approach is possible for imaging with the axial- or 3D-STED OFF-switching pattern. Here, the laser OFF-switching intensities spread over a larger volume compared to the 2D-STED pattern, see section 1.3. A reduction of the light dose which is applied to the marker molecules can hence result in a strong bleaching reduction.

The effect of a lower bleaching per imaged frame when decreasing the scan range in the $z$-direction is represented in figure 4.3 (A). Data was recorded with an axialSTED OFF-switching intensity distribution only for different scan ranges along the optical axis $z$ and a constant scan range of $5 \mu \mathrm{m}$ in the lateral $(x)$ direction. The sample consisted of immunolabeled microtubules of Vero cells. The STED resolution in the $z$-direction was set to $\sim 80 \mathrm{~nm}$. When confining the scan range along the optical axis to $200 \mathrm{~nm}$, the bleaching is reduced by more than 10 -fold compared to a scan with a $1500 \mathrm{~nm}$ range. Here the main reduction of bleaching is caused by the smaller overall amount which is applied to the sample plane (intermediate MINFIELD). Only a little bleaching reduction is expected due to light dose reduction by the OFF-switching pattern shape. When additionally restricting the scan size in the lateral direction to a sub-diffraction extent (scan area fits into the vicinity of the 3D-STED minimum), an even stronger bleaching reduction is expected. A confocal (left) and 3D-STED MINFIELD image (right) of DNA origami labeled with ATTO $647 \mathrm{~N}$ is shown in figure 4.3 (B). The origami consisted of two spots designed to be separated by $91 \mathrm{~nm}$ from spot center to spot center with an orthogonal orientation to the coverslip surface, structure according to [103. The scan area size of the imaged plane by MINFIELD is $190 \mathrm{~nm}$ in the lateral direction $(x)$ and $300 \mathrm{~nm}$ in the direction of the optical axis $(z)$, the pixel size is $10 \mathrm{~nm}$. The STED power distribution for 3D-STED imaging was 30\% for the 2D-STED OFF-switching pattern and $70 \%$ for the axial-STED pattern, featuring an almost isotropic resolution in each spatial direction at high STED resolutions [57. Due to the smaller effective fluorescence spot in STED imaging, the fluorescence signal is lower compared to

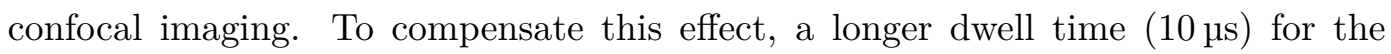
STED acquisition in respect to the confocal dwell time $(1 \mu \mathrm{s})$ was chosen. Scanning along the $z$-direction was performed with a piezo stage (see section 3.1 for precise repositioning. The combined return and settling time of the piezo stage was $300 \mathrm{~ms}$, allowing a distortion-free and precise rescanning of the same sample area. Ten individual $x y$-frames were summed up for (B).

The line profile data of (B) is shown in figure 4.3 (C) (orange and blue dots). The STED data is fitted with a double-peak Gaussian. Accordingly, the (isotropic) resolution is measured to be $\sim 60 \mathrm{~nm}$ along the optical axis (FWHM). The same sample was also successfully recorded with a MINFIELD volume which was repeatedly scanned with 3D-STED, featuring a side length of $190 \cdot 190 \cdot 300 \mathrm{~nm}^{3}$ 

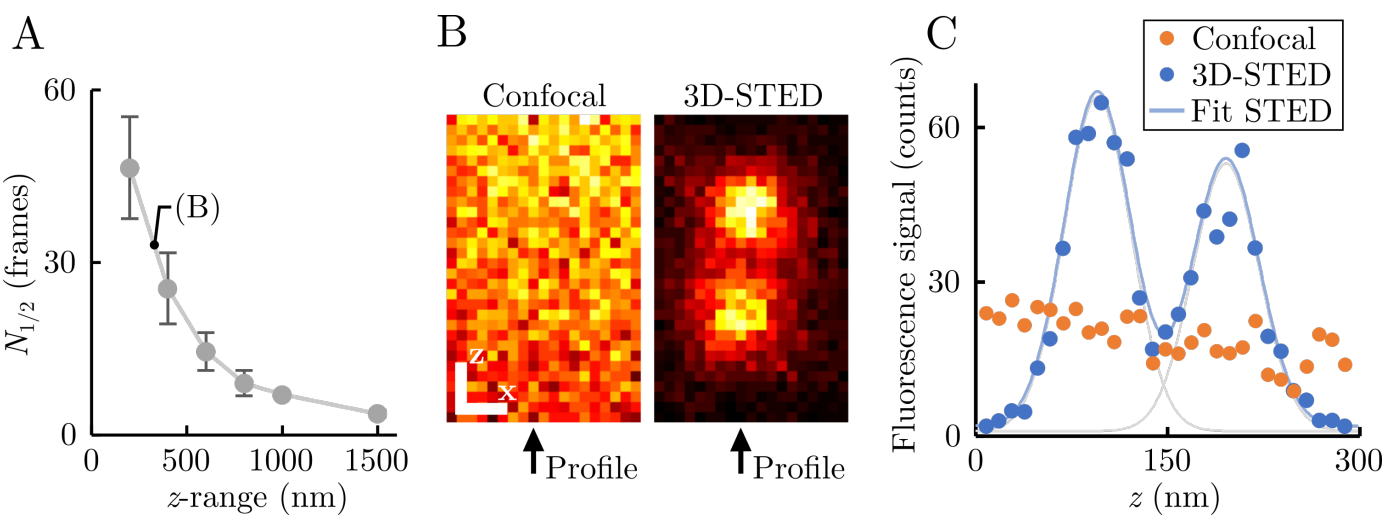

Figure 4.3.: 3D-STED MINFIELD with isotropic resolution. (A) Number of acquired frames before the fluorescence signal is decreased to half the initial value, depending on the scan size along the optical axis. Grey line is a guide to the eyes. (B) Confocal (left) and 3D-STED MINFIELD (right) images demonstrated on DNA origami. Two spots (labeled with ATTO 647N) are designed to be separated by $91 \mathrm{~nm}$ in the axial direction, oriented orthogonal to the coverslip surface 103 . When comparing both images, the resolution increase with STED becomes obvious. (C) Line profiles (as marked in (B)) of the confocal and STED image. A two-peak Gaussian fit indicates a STED resolution along the optical axis of $\sim 60 \mathrm{~nm}$ (FWHM). Scale bars $(x, z)$ are $50 \mathrm{~nm}(\mathrm{~B})$.

$(x, y, z)$. The volume data set of the origami is rendered in appendix A.5.

Since the chosen 3D-STED power distribution features an isotropic resolution, for the first time a resolution of $\sim 60 \mathrm{~nm}$ in each spatial direction was achieved with a single objective lens. These measurements were (to my knowledge) up to this date the highest isotropic resolution ever reported with 3D-STED (later on, a higher resolution was shown, see section 5.6.3. 
4.5. MINFIELD in the green dye spectrum

\subsection{MINFIELD in the green dye spectrum}

MINFIELD has been so far only reported for the red dye spectrum using a STED laser with a wavelength of $775 \mathrm{~nm}[1]$. However, there is a great potential for enhanced performance in the greenish dye spectrum, with a STED laser wavelength of around $595 \mathrm{~nm}$. The dyes, fluorescent proteins and marker molecules in this spectral region tend to bleach faster compared to the red spectrum, hence the resolution is typically significantly lower. A reduced bleaching would be beneficial.

In this section, MINFIELD measurements with an excitation of $485 \mathrm{~nm}(\sim 120 \mathrm{ps})$ and a STED laser at $595 \mathrm{~nm}(\sim 800 \mathrm{ps})$ were performed. The fluorescence detection was between $500 \mathrm{~nm}$ and $550 \mathrm{~nm}$. The other parts of the microscope follow the same design as reported in section 3.1 .

The bleaching reduction by MINFIELD for the dye Oregon Green 488 (ThermoFisher, Germany) is shown in figure 4.4 labeling the nuclear pore complexes NUP153 in Vero cells. MINFIELD images with different side lengths are presented in figure 4.4 (A). The signal was summed up over the first ten frames. Individual lookup tables are used in (A) for a clear representation of the data. The image signal increases inversely with the MINFIELD size.

In figure 4.4 (B), the measured bleaching reduction by scanning smaller fields is plotted (blue dots). The mean fluorescence signal is the sum of the frames before the signal has dropped to $75 \%\left(N_{3 / 4}\right)$ of its starting value. The relatively large standard error ( \pm 1 std. dev.) is mainly due to a strong variation in the brightness of the nuclear pore complexes. Each data point is the mean value of 40 independent measurements originating from two different samples. When scanning a small MINFIELD, the signal is much higher compared to larger fields. However, the signal improvement is lower than expected. The theoretical geometric bleaching reduction (orange dots) is a result of the lower light dose which scales inversely quadratic with the MINFIELD dimension. The data points are normalized to the measured fluorescence signal for a MINFIELD scan area size of $300 \mathrm{~nm}$. In contrast to the red dye spectrum, the deviation between the theoretical and experimental trend at small scan areas indicate a non-linear dependency between excitation and STED light. Important to note, these results are observed for the dye Oregon Green 488 and may significantly vary for other dyes or proteins. Nevertheless, even if the MINFIELD effect is not as significant as for the red dye spectrum, still a much higher fluorescence signal is observed for small scan areas. The signal gain was $\sim 16$-fold between a scan area size of $300 \mathrm{~nm}$ and $50 \mathrm{~nm}$. 
A

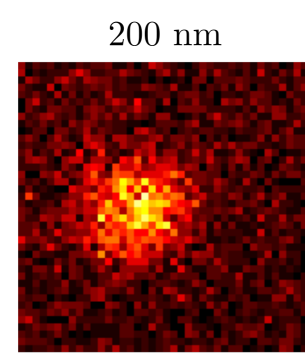

counts

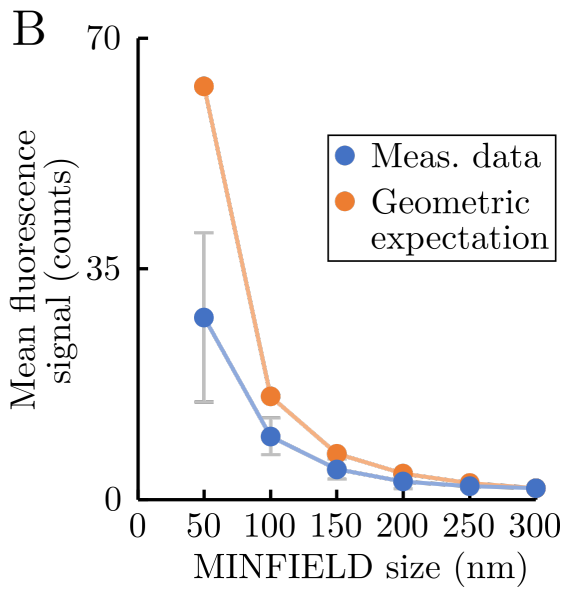

Figure 4.4.: Bleaching reduction by MINFIELD for Oregon Green 488 on nuclear pore complexes (indirect immunolabeling, NUP153). (A) Representative images of the nuclear pore complexes with different MINFIELD sizes. The fluorescence signal was accumulated over ten frames. Lookup tables are $(0-27)$ $200 \mathrm{~nm},(0-32) 150 \mathrm{~nm},(0-77) 100 \mathrm{~nm}$, and (0 - 73) $50 \mathrm{~nm}$. (B) Measured mean fluorescence signal (blue dots) for different MINFIELD sizes. Lines are a guide to the eyes. The orange dots represent a linear scaling with the inverse MINFIELD area, and are normalized to the $300 \mathrm{~nm}$ field size. At smaller MINFIELD sizes, the fluorescence signal is significantly higher compared to larger fields. The geometric reduction of the light dose by scanning fewer pixel shows a stronger theoretical bleaching reduction as observed in the experiment. 


\section{Adaptive-Illumination STED Nanoscopy}

In this chapter, a novel concept is presented where high STED powers are only applied to areas of the sample where they are needed to extract high spatial information. To locate these areas, the sample is iteratively probed with increasingly higher STED powers (and resulting resolutions). After each resolution increase, the structure of interest is spatially located to a higher extent. In the end, the final (very) high resolution STED image is only acquired at this prelocated areas (where structure is present). The overall light dose is reduced, and in the best case the excess OFF-switching intensities of the doughnut are sidestepped to the structure, whereby photobleaching is strongly reduced.

\subsection{Introduction}

In a typical STED microscope, the excitation focal spot is overlayed with a STED focus intensity distribution shaped like a doughnut in the lateral direction 15,78 , 104. The photobleaching of the used fluorophores, which is mainly induced by excess intensities at the crest doughnut [89], has prohibited STED fluorescence nanoscopy to achieve molecular resolution. Depending on the resolution (STED power), the OFF-switching intensity at the crest may be manyfold higher than the actually needed intensity for $\mathrm{ON} / \mathrm{OFF}$-state separation close to the doughnut minimum. Since the high photon flux density is mainly the reason for fluorophore bleaching, some techniques have been developed to either lower the number of photons which are applied to the fluorophore or to protect it from the negative effects of the photons. The concept which is known as „multiple off-state transitions“(MOST) uses the low light intensity OFF-switching (also known as RESOLFT [105] [106]) to transfer the fluorophores into a state where they do not interact with the STED photons. The bleaching is strongly reduced, allowing to record a higher number of frames at the same sample region [107]. A recently published concept called MINFLUX [46] is able to achieve molecular resolution by probing of single ON/OFF-switched fluorophores with an excitation pattern formed like a doughnut. In MINFLUX, the fluorophore is only excited with parts of the doughnut which are close to the minimum, while 


\section{Adaptive-Illumination STED Nanoscopy}

the higher intensities away from the minimum are avoided. Another concept is RESCue STED [108 which has the same basic idea as controlled light exposure microscopy [109]. In RESCue the overall sample illumination is reduced by shortly probing for fluorophore-free sample regions during the STED image acquisition. If not enough fluorecence photons are collected in a defined fraction of STED image pixel dwell time, illumination is stopped immediately to save the light dose of the remaining pixel dwell time. Very recently, a method which is called MINFIELD [1] was presented (see also chapter 4). Here, the scan field (image size) is limited to a small sample region (typically $50-200 \mathrm{~nm}$ ) which roughly corresponds to the dimension of the minimum of the doughnut. The high OFF-switching intensities of the doughnut crest are sidestepped to the fluorophores of interest. With MINFIELD, more frames of the same sample regions can be acquired, or at a higher spatial resolution, because the lower bleaching can be used to apply higher STED intensities. The drawback of this method is the limitation to only a very tiny sample area. Surrounding sample areas exposed to the STED intensities of the doughnut crest cannot be imaged since they get bleached 1 .

\subsection{Concept of DyMIN}

In this section, a novel illumination concept is explained which adapts the STED resolution in response to the sample fluorophore distribution, namely Dynamic intensity MINimum (DyMIN). The sample fluorophore distribution is iteratively probed at increasingly higher resolutions, and the illumination is stopped when not enough fluorescence is detected. DyMIN saves overall unnecessary OFF-switching photons, and sidesteps the excess doughnut intensities to the structure without a limitation to only small sub-diffraction scan areas.

For a concept explanation, two fluorophores which are spaced closer than the diffraction limit are assumed, see figure 5.1 (A). Somewhere away from the fluorophores the scan starts with a confocal resolution (without any STED light). When one of the dye fluorophores is first hit by the low intensity of the Gaussianshaped excitation focus, the dye will emit fluorescence (which is detected) with a light level following the shape of the excitation (with a linear excitation behavior). Now some STED light is applied, but only a little, such that the dye molecule again is in the OFF-state. At a later scan position, the dye molecule again will transfer into the ON state because it is again excited, now by the in the present case effective fluorescence PSF. And again the resolution is increased until no fluorescence is detected. This proceeds to a predefined final target resolution (STED power). In an analog way, the DyMIN scan continues when it moves away on the other side of the fluorophore assembly. As soon as no fluorescence is detected, the STED power is lowered until again some signal is present, and so on. 

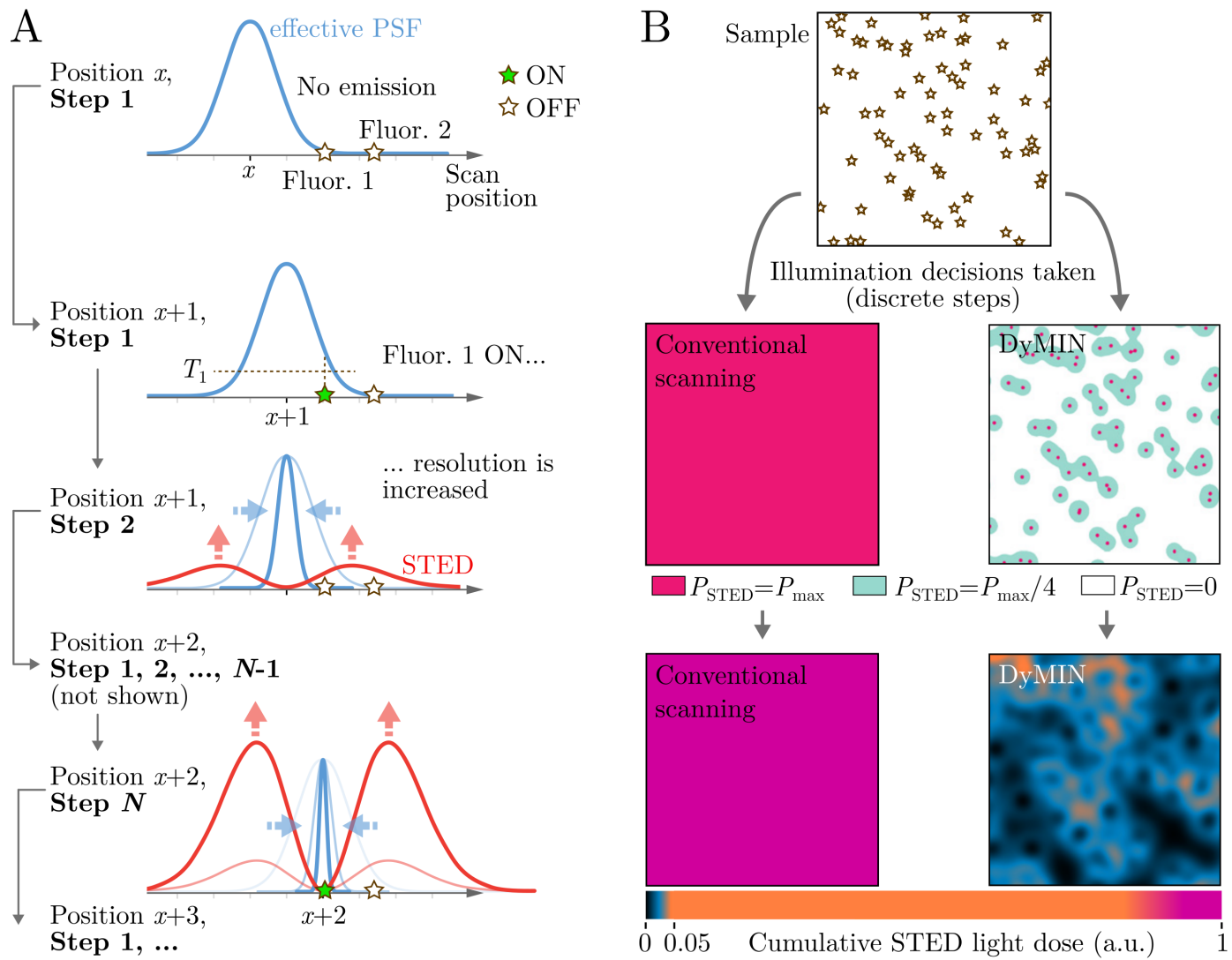

Figure 5.1.: STED fluorescence nanoscopy with a Dynamic intensity MINimum (DyMIN) (A) Scan scheme for two markers closer than the diffraction limit. At every scan position, the fluorescence signal is probed with increasing resolutions, starting at diffraction-limited (confocal) resolution $(P=0$, top), and subsequently with higher resolutions $(P>0)$. If at a scan position, at any probing resolution, no signal is detected (no marker present in the effective focus, $T_{i}$ is not reached), the further illumination steps are aborted. The scan continues to the next position, starting again to probe at confocal resolution. If the detected signal at a step is above a threshold (e.g. $T_{1}$, second row), the STED power is increased (higher resolution, third row). If fluorescence signal is still present, the resolution is increased further and so on, until a final resolution (corresponding to the STED power $P_{\max }$ ) is reached. In the best case, the highest STED power (resolution) is only applied when the minimum of the doughnut is centered above (a) fluorophore(s) vicinity (fourth row). (B) Shown is a simulated sample with randomly distributed fluorophores (top). Because different STED powers were used in each pixel (middle) for conventional scanning (left) and DyMIN scanning (right), a dramatic difference in the light dose which is applied to the sample is observed (bottom). For a conventional scan, the maximum STED power $P_{\max }$ is used at each scan position. With DyMIN scanning, sample areas are imaged without STED light (white), or with little (probing) STED light (green). Only in a few areas (where fluorophores are present) $P_{\max }$ (pink) is needed to extract the final resolution. In this example, the cumulative light dose acting at the fluorophores is $\sim 45$ times lower with DyMIN compared to conventional scanning. Figure reproduced from ref. [2]. 


\section{Adaptive-Illumination STED Nanoscopy}

Thereby DyMIN avoids scanning the intense (full power) doughnut crest above the dyes as much as possible. In DyMIN scanning, the samples (and the fluorophores) are on average illuminated with a lower STED light dose compared to a conventional scan (always the highest resolution), simply because the STED power is not at the maximum for all the time. In fact, the maximum resolution is applied only at scan positions within a distance to the fluorophore smaller than approximately the distance from the doughnut center to the point where the required threshold intensity for OFF-switching is reached. By that, a MINFIELD-like condition is generated, but with the dynamic adjustment of the resolution, the limitation to only small scan fields is lifted while avoiding to scan the highest intensity doughnut across the complete sample. In other words, DyMIN scanning localizes fluorophores in lowand medium-resolution images. The positions of the fluorophores in these images render an illumination mask for the sample positions where the maximum STED power needs to be applied.

It is important to note that with a denser fluorophore distribution like in a realistic sample, DyMIN scanning can still lower the overall exposure. Mainly because the STED power is not set to the maximum all the time (if sample space without fluorophores is present). In an optimal case not only the overall light dose is lower (which is important for live-cell applications) but the fluorophores are not subjected to the excess OFF-switching intensities (MINFIELD condition). The light dose of DyMIN is typically lower when compared to RESCue STED, whereby the dye molecules are illuminated with the maximum STED power (and also with the intense doughnut crest) during the decision time of the STED probing step. Another drawback of RESCue is the relatively long integration time before an appropriate decision (the fluorescence signal is Poisson-distributed) can be made. This is due to the low fluorescence photon flux at high STED resolutions. In consequence, the effectiveness of RESCue gets worse at high resolutions, but especially at these high STED powers a bleaching reduction starts to be important.

In DyMIN, discrete pixels are scanned, and it is reasonable to also adjust the STED power during the scan in discrete levels while probing for the sample fluorophore distribution. Another reason for discrete steps is the necessity to collect a certain amount of fluorescence photons to make a decision on the presence or absence of fluorophores.

For DyMIN, the following formalized practical implementation is defined: in each pixel, a sequence of up to $i=1, \ldots, N$ different imaging parameters is performed while the fluorescence signal is registered for a time $t_{i}$. The fluorescence results from the illumination with a Gaussian excitation focus and the STED OFF-switching pattern $h_{i}^{S T E D}$. The variable STED power $P_{i}$ is from the continuously growing ordered list $\left\{P_{1}=0, P_{2}, \ldots, P_{N}=P_{\max }\right\}$, with $P_{\max }$ as the final STED power. If 
at any step $i$ the detected fluorescence signal does not exceed a signal threshold $T_{i}$, the illumination of the excitation and STED is directly stopped. The photon count of the specific pixel is set to zero, since no final high resolution image is acquired (no fluorophore is present per definition). However, if the threshold $T_{i}$ is reached, the illumination proceeds with the step $i+1$, i.e. collecting signal for the time $t_{i+1}$ with the higher STED power $P_{i+1}$. If at any step sufficient signal is collected, the process continues until $P_{\max }$ is reached, and only these pixels contribute to the final high resolution image (in this first DyMIN implementation). A mask $m_{i}(x, y)$ which includes the illumination decision if the STED power $P_{i}$ was applied at a step $i$ can be written as

$$
m_{1}(x, y)=1 \forall x, y \quad ; \quad m_{i+1}(x, y)=\theta\left[s(x, y) \otimes h_{i}^{e f f}(x, y)-T_{i}\right]
$$

whereby $s$ is the fluorophore distribution (the sample structure), and $h_{i}^{\text {eff }}$ the effective fluorescence distribution (the ON-state in focus) in the presence of excitation and a certain STED power $P_{i}$. The convolution operator is $\otimes$ and the Heaviside step function is $\theta$. The STED light dose (product of the STED intensities and illumination times) which accumulates at any position $x, y$ is

$$
D_{t o t}(x, y)=\sum_{i} m_{i}(x, y) \otimes h_{i}^{S T E D}(x, y) \cdot t_{i}
$$

Equation 5.1 and 5.2 are both evaluated in tabular form in table 5.1 in section 5.3 . The effectiveness of DyMIN scanning strongly depends on the choices of the parameters $t_{i}, T_{i}$ and $P_{i}$ which are independent and need to be set for each step $i$ (excluding step $N$ ). For a 4-step DyMIN scan (three probe steps and the final image step) nine appropriate values are required, whereby $P_{0}=0$. The best choice for the parameters is highly dependent on the sample structure, and also on the bleaching kinetics of the fluorophore. However, a few assumptions can be made.

The STED resolution (the FWHM diameter of the effective PSF $h_{i}^{\text {eff }}$ ) is related to the OFF-switching power in $h_{i}^{S T E D}$ by a square-root dependency. Further, the spatial area of the effective PSF has also a square-root dependency on the diameter of the effective PSF. Consequently, the effective probing area has an inverse dependency on the STED power to the power of four. The initial application of a little OFFswitching light (with the starting point $h_{1}^{S T E D}=0$ ) leads to a relatively high increase of the resolution, and thus decrease of the probing area. In DyMIN scanning, selections of $P_{i}$ are useful where the slope of the power-resolution curve is steep. Here, by adding only a little STED light, much more spatial information about the fluorophore distribution can be obtained. In general, a comparatively great subset of the probing steps should use STED powers $P_{i}$ of this area, which includes a probe 


\section{Adaptive-Illumination STED Nanoscopy}

step with confocal resolution as the first step.

Counte-intuitively, adding more DyMIN scan steps typically results in a lower total light dose applied to the sample and fluorophores. This is because with more probing steps, the area where high STED powers are used is smaller. However, the reduction of the light dose on the fluorophores when the number of probing steps $N$ is increased asymptotically slows down (Figure 5.2).

\subsection{Simulation framework}

In this section, the framework for the DyMIN simulations is presented. The sample structure (object) was rendered by dots with discrete positions. The object was convolved by a Fast Fourier Transformation (FFT) for each step $i$ with the effective fluorescence PSF $h^{e f f}\left(P_{i}\right)$, corresponding to the probe step STED power $P_{i}$. The scaling of the resolution in dependence on the applied STED power $P$ was experimentally ascertained. To this end, the resolution at small fluorophore clusters was evaluated for different powers and the data points were fitted by a least mean square algorithm. For ATTO $647 \mathrm{~N}, P_{s} \approx 2.464 \mathrm{~mW}$ for the microscope used. The convolution result was transferred onto the pixels of the virtual scan. The pixel values were either set to zero (low) or one (high), depending on whether the pixel value exceeds the threshold of $1 / e$ of the signal level. The resulting binary pixel mask $m_{i}$ is the illumination map for the next step $i+1$. Here, the illumination is only applied at the high pixels of $m_{i}$, using the doughnut $h^{S T E D}\left(P_{i+1}\right)$ with the higher STED power $P_{i+1}$, with the longer integrated decision time $t_{d e c}\left(P_{i+1}\right)$ due to the lower fluorescence signal at the higher STED resolutions.

The calculation of the decision time, which is needed for a certain resolution, is based on decision times which were observed for relevant resolutions in STED. One is $10 \mu \mathrm{s}$ for a $50 \mathrm{~nm}$ resolution, which is a resolution close to the optimum for the intermediate step (with a final resolution of $25 \mathrm{~nm}$, found during bleaching experiments and simulations, see figure 5.5). The other is $30 \mu \mathrm{s}$ at a resolution of $25 \mathrm{~nm}$, to match the decision time which was experimentally found for RESCue at this resolution. When taking the geometrical dependence of the fluorescence signal on the resolution into account, equation 5.3 can be used to calculate the decision time in $\mu$ s for different STED resolutions res:

$$
t_{d e c}=\gamma \frac{n R e s^{2}}{r e s^{2}}+\delta
$$

$n$ Res $=50 \mathrm{~nm}$ is the nominal resolution to fit the curve with both fitting parameters $\gamma=2 / 300000 \mathrm{~s}$ and $\delta=1 / 300000 \mathrm{~s}$. 
Table 5.1.: Simulation framework to calculate the STED light dose which is applied during DyMIN scanning. Mask $k_{i}$ corresponds to $m_{i}$ in the text, Dose to $D$.

\begin{tabular}{|c|c|c|}
\hline Step & Mask creation & STED light dose \\
\hline 1 & $h^{e f f}\left(P_{1}=0\right) \otimes S t r u c t \rightarrow$ Mask $_{2}$ & Dose $_{1}=0=$ Mask $_{1} \otimes h^{S T E D}\left(P_{1}=0\right) \cdot t_{d e c}\left(P_{1}\right)$ \\
\hline 2 & $h^{e f f}\left(P_{2}\right) \otimes S t r u c t \rightarrow$ Mask $_{3}$ & $\operatorname{Dose}_{2}=$ Mask $_{2} \otimes h^{S T E D}\left(P_{2}\right) \cdot t_{d e c}\left(P_{2}\right)$ \\
\hline 3 & $h^{e f f}\left(P_{3}\right) \otimes S t r u c t \rightarrow M_{a s k}$ & $\operatorname{Dose}_{3}=$ Mask $_{3} \otimes h^{S T E D}\left(P_{3}\right) \cdot t_{d e c}\left(P_{3}\right)$ \\
\hline$\ldots$ & & -. \\
\hline$N$ & & $\operatorname{Dose}_{N}=\operatorname{Mask}_{N} \otimes h^{S T E D}\left(P_{N}\right) \cdot t_{d e c}\left(P_{N}\right)$ \\
\hline Total & & Dose $_{\text {tot }}=$ Dose $_{2}+$ Dose $_{3}+\ldots+$ Dose $_{N}$ \\
\hline
\end{tabular}

The spatial distribution of the light dose in an DyMIN image is highly heterogeneous (since the extended doughnut profile is not irradiated at any sample position) as intended by the method (low dose on the fluorophores). To calculate the spatial dose arising due to the probe steps, the pixelated $m_{i}$ is convolved (FFT) with the OFF-switching pattern (doughnut) light exposure $h^{S T E D}\left(P_{i+1}\right) \cdot t_{i+1}$. The highest dose typically occurs from the final step $N$, which is computed using the $m_{N-1}$ and the light exposure of the doughnut $h^{S T E D}\left(P_{N}\right)$ times the final dwell time $t_{i m g}$. The spatially dependent $D_{\text {tot }}$ is the sum of all dosages $D_{i=2}$ up to $D_{N}$. For a clearer understanding, the described simulation routine is presented in table 5.1 for $N$ steps.

The relative STED light dose on the structure is the mean of the individual values of $D_{\text {tot }}$ which acts directly at the fluorophore positions (simulated dots). The DyMIN light dose was always normalized to the light dose of a corresponding conventional scan.

As mentioned, for DyMIN scanning the ordered list from $i=1$ to $N$ is processed either to the end or the illumination is stopped at a step $i$ if the threshold is not reached. For each simulation (or measurement), the last step which was performed is saved into an "illumination map". If the pixel illumination was stopped at a step $i$, apparently the step $i-1$ and all previous steps have been applied. Therefore the pixelated illumination map of the last applied step carries the complete information of the illumination.

The times fluorescence signal is integrated at any probing step $i<N$ during DyMIN scanning, are defined as decision times. The integration time which is spent to collect the signal in the final step $N$ is defined as the dwell time of the scan. However, the overall time the scan rests at a voxel or pixel is the sum of the dwell time, each decision time and the rise and fall time of the beam control which is needed to adapt the STED power or to block the beams. The overall time of a DyMIN scan is typically much longer compared to the conventional counterpart. Partly because 


\section{Adaptive-Illumination STED Nanoscopy}

the decision times add some tens of microseconds, but mainly because the reduced bleaching results in an accordingly longer dwell time.

\subsection{Assessment of DyMIN}

To compare the light dose of different scan methods, simulations and experiments have been performed. DyMIN scanning is simulated to find the best parameters for the probing steps and to compare it with conventional scanning and RESCue. The simulations are written in Python 3.6. For the simulation, the STED light dose acting directly on the fluorophores is evaluated for a variety of sample structures (Figure 5.3 in section 5.4.1). The simulation framework is also used to evaluate the overall light dose reduction on the sample for the DyMIN imaging experiments. For the 2D-STED experiments, additionally the dose reduction directly on the structures/fluorophores is calculated.

Different structures were simulated in a square region which features a side length of $5 \mathrm{~mm}$. The samples consisted of (i) randomly distributed single points (like e.g. fluorophores), (ii) randomly arranged straight lines (like e.g. cytoskeletal filaments), and (iii) randomly arranged circles with varying diameter (like e.g. sub-diffractionsized biological structures, i.e. NPCs, vesicles, viruses or synapses), see section 5.4.1. For conventional scanning, the light dose exposure of each pixel was calculated by convolving (FFT) every pixel with the OFF-switching intensity profile corresponding to the final STED power $P_{\max }$ accounting for the acquisition time $t_{i m g}$ of the final STED step (100 $\mu$ s, as a good practical value).

For DyMIN scanning, first the best parameter set was calculated by minimizing the accumulated OFF-switching light dose which acts directly on each fluorophore. The thresholds $T_{i}$ for the simulations were set to $1 / e$ of the maximum of the fluorescence signal. Since the first probing step is at confocal resolution, the only parameters to optimize are the resolutions of the intermediate steps (intermediate: steps between the first (confocal) and final high-resolution STED step $N$ ). With this optimized parameter set, the DyMIN light dose is calculated. The simulation framework is also capable to simulate RESCue STED, using only one probe step at the final resolution according to $P_{\max }$. This allows to compare RESCue with conventional scanning and different variants of DyMIN (different number of steps).

The simulations show that already a 3-step DyMIN scan of the sample type (i) with OFF-switching powers of $0, P_{\max } / 4$, and $P_{\max }$ (confocal resolution, half of the final resolution, and the final high resolution $(25 \mathrm{~nm})$, respectively) can lower the accumulated light dose per fluorophore by a factor of $\sim 45$ when compared to conventional scanning (see Figure 5.1(B)). In this case, DyMIN is able to achieve a $\sim 3.2$-fold lower light dose compared to RESCue. As mentioned, at a larger number of DyMIN steps the light dose on the fluorophores can be reduced further (see Figure 
5.2. The improvement slows down for a higher number of intermediate DyMIN steps, as found by simulations and measurements. After a certain high number of intermediate steps, the light dose is expected to rise, due to a lower chance of empty probing pixel at higher step numbers. The probing resolution parameter sets for figure 5.2 are shown in table 5.2 .

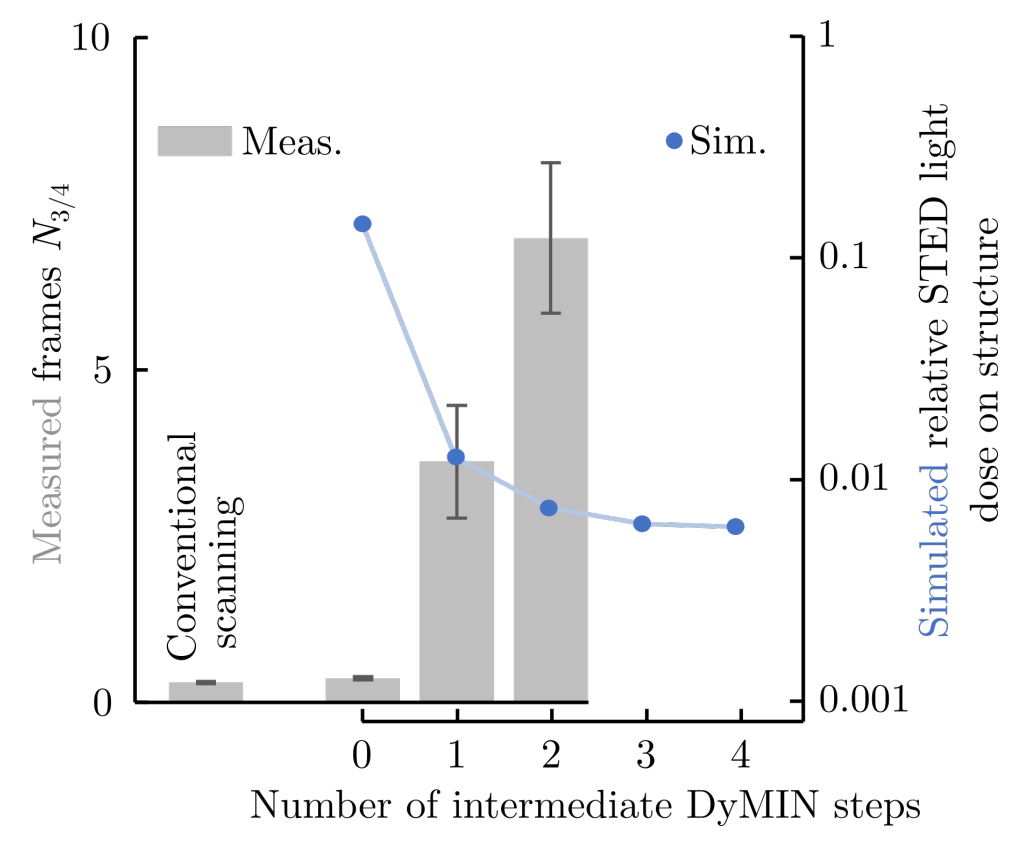

Figure 5.2.: Influence of the number of intermediate DyMIN steps. Gray bars: measured frame number $N_{3 / 4}$ after which the fluorescence level is dropped to $75 \%$ of the initial value vs. the number of intermediate probing steps of the DyMIN scan. Intermediate: the probing steps without the confocal probing step and the final resolution (image) step. The final resolution was $50 \mathrm{~nm}$, the dwell time $100 \mu \mathrm{s}$, and the sample consisted of nanoassemblies (Bead R, GattaQuant). Blue dots represent the simulated STED light dose acting directly on the structure for a similar sample and imaging parameters vs. the intermediate probing step number. The blue line is a guide to the eyes. For $>3$ intermediate steps the simulated light dose almost arrives at a minimum, and may rise for higher numbers. Figure reproduced from ref. [2].

The reduced probability for photobleaching is connected to the reduction of the light dose in dependence on the specific bleaching kinetics of the particular dye. A possible non-linear bleaching behavior on the OFF-switching light or on prior light exposure can lead to a bleaching reduction which is greater than the factor of the light dose lowering by DyMIN. However, minimizing the STED light dose directly 
Table 5.2.: Resolutions used for figure 5.2 for the different DyMIN scans. Resolutions were optimized for a density of $2 / \mu^{2}$ and a final resolution of $50 \mathrm{~nm}$.

\begin{tabular}{llllll}
\hline Step & 2-step & 3-step & 4-step & 5-step & 6-step \\
\hline 1 & $230 \mathrm{~nm}$ & $230 \mathrm{~nm}$ & $230 \mathrm{~nm}$ & $230 \mathrm{~nm}$ & $230 \mathrm{~nm}$ \\
2 & - & $70 \mathrm{~nm}$ & $100 \mathrm{~nm}$ & $135 \mathrm{~nm}$ & $175 \mathrm{~nm}$ \\
3 & - & - & $50 \mathrm{~nm}$ & $80 \mathrm{~nm}$ & $120 \mathrm{~nm}$ \\
4 & - & - & - & $50 \mathrm{~nm}$ & $75 \mathrm{~nm}$ \\
5 & - & - & - & - & $50 \mathrm{~nm}$ \\
\hline
\end{tabular}

on the fluorophores should also feature the lowest bleaching [1].

As experiments and simulation show (Figure 5.2), further improvement of the light dose reduction is only a little for DyMIN scans with $N>4$ steps. Another fact disfavors higher DyMIN step numbers: the decision confidence if a threshold is reached (Poisson statistics of the fluorescence signal come along with an uncertainty) increases with the signal collected at any probing step. Because the signal is lower at higher resolutions, a much longer decision time is needed which results in a higher total light dose. For each decision (if to continue illumination), a finite probability exists for a wrong decision due to the Poisson statistics (further pixel illumination is aborted even though the pixel has enough fluorophores). More DyMIN steps increase the overall probability that a pixel is falsely imaged.

\subsubsection{Simulated light dose reduction for different samples}

As described in section 5.4, three basic structure geometries have been simulated with a 3 -step DyMIN scan (Figure 5.3). Those were dots (left), lines (middle) and rings (right), representing basic structure geometries of biological samples. Dots: central channel of nuclear pores, single fluorophores etc.; Rings: clathrin, virus particles, synapses, gephyrin etc.; Lines: vimentin, tubulin etc.

The lines and rings were rendered by single points with a spatial distance much closer than the resolution of the DyMIN step $N-1$. Since individual points are not resolved by that step, the structure appears quasi-continuous. For all samples the structure was generated randomly. For the simulations in this section, similar imaging parameters were used. The resolution at the final step $N$ was $25 \mathrm{~nm}$, the dwell time was $100 \mu \mathrm{s}$, and the pixel size was $15 \mathrm{~nm}$ (slightly to large, but does not affect the results). For RESCue a decision time $t_{d e c}$ of $30 \mu$ s was selected. 

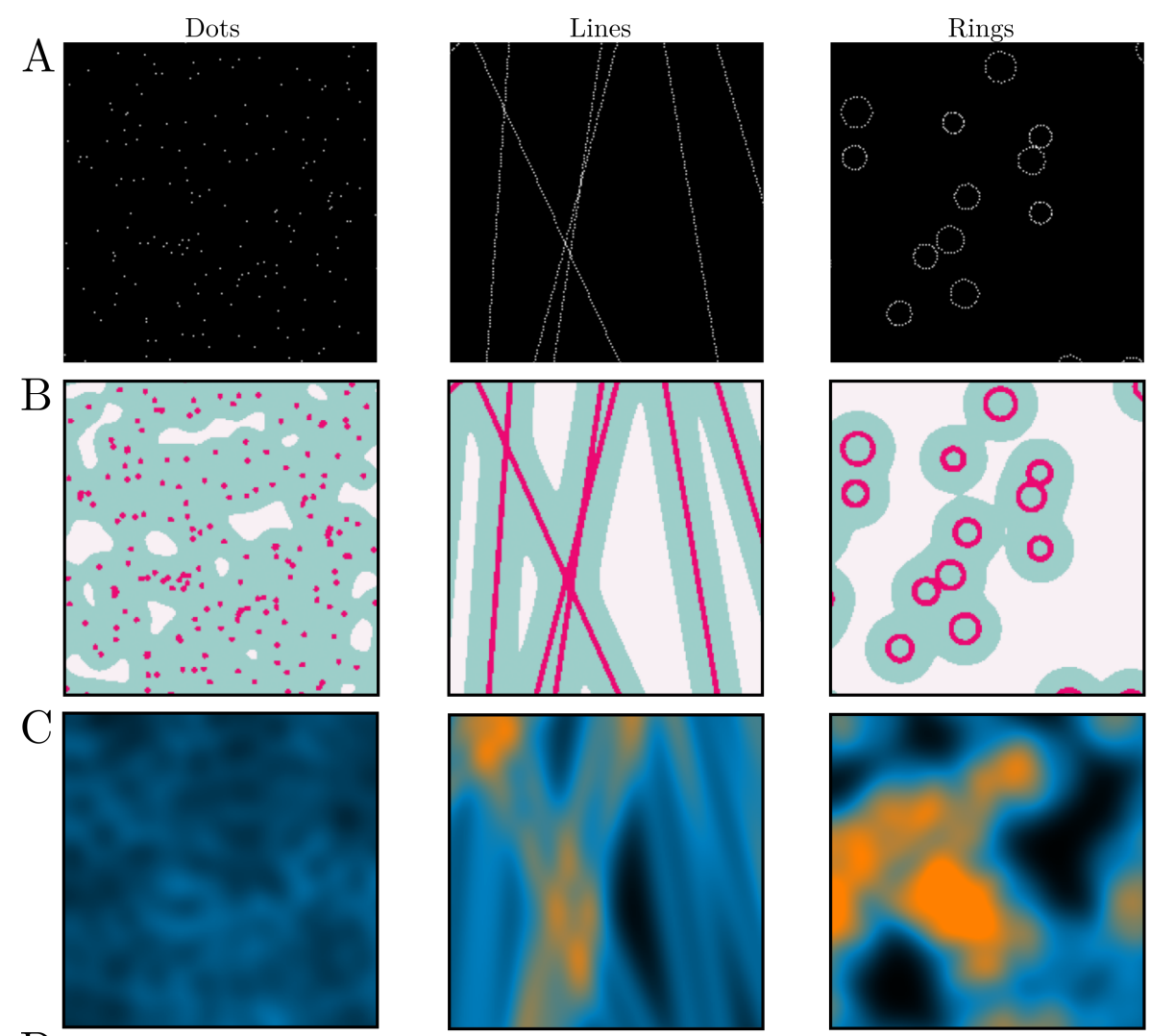

$\mathrm{D}$

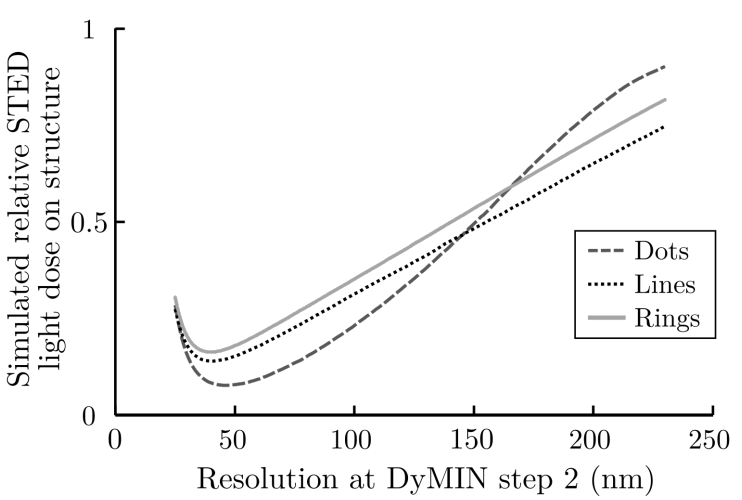

0.3

Norm. cumulative STED light dose

Figure 5.3.: Simulated DyMIN scan for different structure geometries. 3step DyMIN, the final resolution was $25 \mathrm{~nm}$, dwell time $100 \mu \mathrm{s}$. Left column: single spots (density of 20 points / $\mathrm{\mu m}^{2}$ ). Middle column: assembly of linear structures. Right column: assembly of ring structures. (A) The specific random fluorophore distribution in the sample. White: pixels including a fluorophore, black: pixels without fluorophore. (B) Illumination map of the STED powers applied throughout the scan. White: illumination stopped after the confocal probing; green: stopped after the intermediate probing; pink: final STED power applied. (C) The spatial STED light dose which originates from the particular DyMIN scan. The dose is highly heterogeneous and much lower than needed for conventional scanning (dose of 1). (D) Shown is the mean STED light dose on the structures in dependence of the intermediate resolution. For the structures shown here, a global minimum exists, demonstrating an optimal intermediate resolution. The optimum for the dots is at $46 \mathrm{~nm}$, lines $40 \mathrm{~nm}$, and rings $39 \mathrm{~nm}$. Figure reproduced from ref. [2]. 


\section{Adaptive-Illumination STED Nanoscopy}

In a 3-step DyMIN process mainly the resolution of the intermediate step (second step) has to be optimized which strongly influences the total light dose on the structure (Figure 5.3 (D)). The first step has a confocal resolution. Here, the intermediate resolution was changed in $1 \mathrm{~nm}$ increments starting at the confocal resolution $(230 \mathrm{~nm})$ up to the final resolution $(25 \mathrm{~nm})$. For all three structures the influence of the intermediate step on the total light dose is shown.

In figure 5.3 (C) the spatial distribution of the total light dose is shown which occurs for the optimal resolution in step 2, as calculated by the minimization. For the structures simulated, a global optimum of the light dose exists with respect to the intermediate resolution. This optimum is relatively broad, which allows a coarse adjustment of the step 2 resolution without a significant performance loss. The optimum appears roughly at half the resolution of the final step for all three structures. The simulations were carried out with a set of scan parameters which are close to the practical application (realistic values). Parameter sets or samples exist which feature no optimum (e.g. where DyMIN has a negative effect), for instance a very short dwell time or very dense samples. However, a useful DyMIN implementation allows to use a much longer dwell time (since bleaching is reduced) which will always reduce the light dose minimum when compared with the conventional scan. In fact, the optimum depth in respect to the conventional scan is the bleaching reduction which is convertible into a higher dwell time (by the same factor) and signal. An appropriate choice of the step 2 resolution should be near the optimum but a little bit lower (further right on the curve in figure 5.3 (D)). Thereby, a kind of clearance area is generated around the sample structure (without much higher bleaching), which assures that the structure will be completely contained in the illumination mask $N-1$.

In figure 5.4 (A) the simulated light dose is plotted for different densities of a dot structure, using the same sample type as in figure 5.3 left column. The sample density was simulated in the range between 0 and 200 points $/ \mu^{2}$. The STED light dose is shown for conventional scanning, RESCue, 2-step DyMIN, 3-step DyMIN and 4-step DyMIN scanning. Here, the same imaging parameters were used as for figure 5.3 (25 nm final resolution). The probing resolution sets for the different DyMIN methods which were used in this density study are listed in table 5.3 .

The resolutions for probing were optimized for a sample density of $20 / \mu^{2}$ and vary if the sample density is different. A different sample density (or type) will most of the time require a different probing resolution set (compared to the one used here) to achieve the lowest light dose possible. In this study it is shown that a 3-step DyMIN scan gives a lower light dose compared to RESCue at sample densities which are in a realistic range, compare figure 5.4 (B). At low densities, RESCue features a high light exposure base line which originates from the fixed (relatively long) decision time while using the highest OFF-switching light power. However, at high densities, 

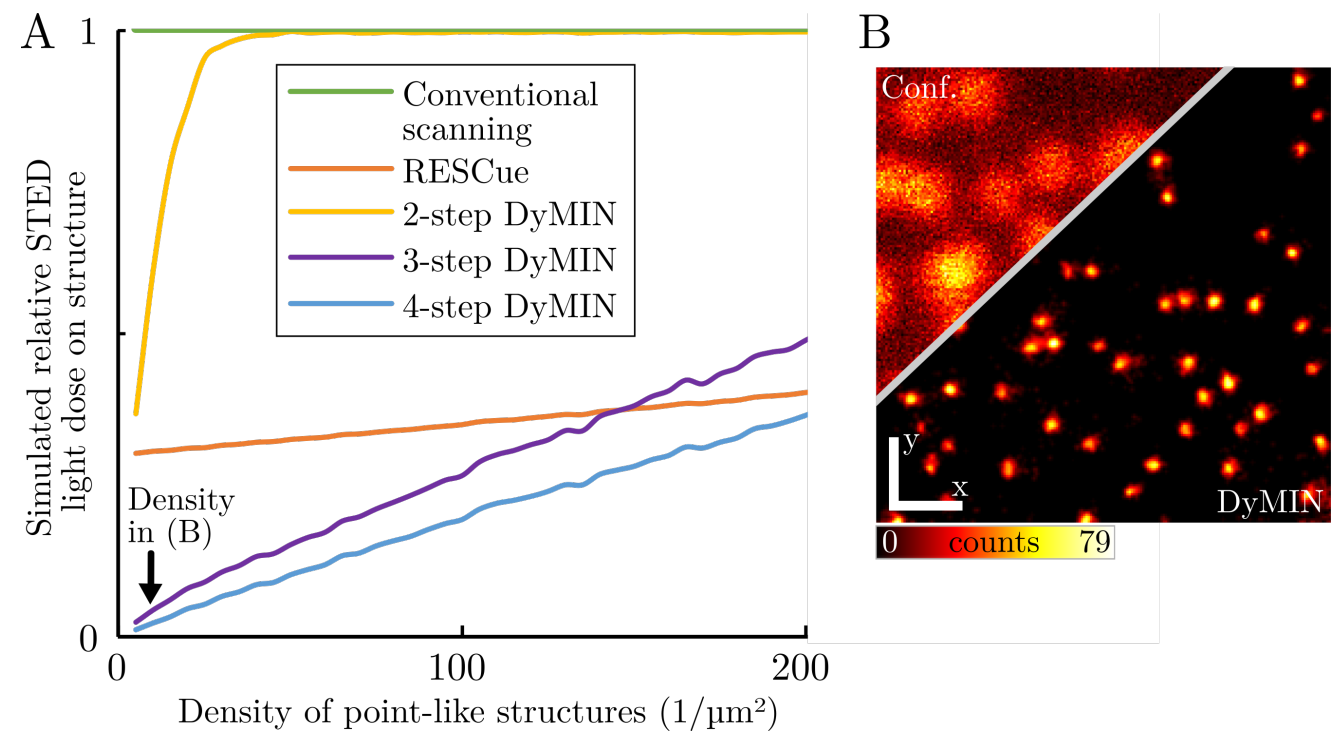

Figure 5.4.: Light dose for different scan schemata in dependence on the sample density. (A) The simulated relative STED light dose on the structure vs. the sample density (dot structure). Final resolution $25 \mathrm{~nm}$, dwell time $100 \mu \mathrm{s}$. Simulated are conventional scanning, RESCue and DyMIN with $2 / 3 / 4$ steps for typical sample densities (e.g. $\sim 5-20$ nuclear pore complexes $/ \mu^{2}$ ). With DyMIN scanning the light dose is more than a magnitude lower compared to conventional scanning. DyMIN parameters were optimized for a density of $20 / \mu^{2}$. (B) DyMIN recording of NPCs (immunofluorescence), featuring a density of $\sim 7 / \mathrm{\mu m}^{2}$ (image is smoothed with a Gaussian of 1.5 pixels). The scale bars $(x, y)$ are $500 \mathrm{~nm}(\mathrm{~B})$. Figure reproduced from ref. [2].

the intermediate DyMIN steps cannot resolve individual dots, in this case RESCue is the better choice due to the higher probing resolution. When comparing 4-step DyMIN and 3-step DyMIN, the 4-step method conceptually provides a lower light dose and is especially well-fitting for very small structures, due to the good chance for empty probing at higher resolutions.

\subsubsection{Experimental parameter optimization}

In this section, the reduction of the photobleaching behavior of fluorescent beads is investigated for MINFIELD, 3-step DyMIN, RESCue, and conventional scanning (Figure 5.5). The experimental data is compared to simulations which remodel the experiments. When comparing the different scan schemata, excitation and STED powers were set to the same level for all methods. $P_{\max }$ of the final DyMIN step was used as the STED power for all other methods. The imaging parameters used for the experiments are listed in appendix A.2.

In figure 5.5 (A) the measured number of frames $N_{3 / 4}$ (which can be recorded before 
Table 5.3.: Resolutions for the different DyMIN methods used in figure 5.4. The resolutions were optimized for a density of $20 / \mu^{2}$ and final resolution of $25 \mathrm{~nm}$.

\begin{tabular}{llll}
\hline Step & 2-step & 3-step & 4-step \\
\hline 1 & $230 \mathrm{~nm}$ & $230 \mathrm{~nm}$ & $230 \mathrm{~nm}$ \\
2 & - & $50 \mathrm{~nm}$ & $105 \mathrm{~nm}$ \\
3 & - & - & $35 \mathrm{~nm}$ \\
\hline
\end{tabular}

the average fluorescence level in the scan area is dropped below $75 \%$ of the initial value) as a function of the resolution of the intermediate probing step is shown. The four data sets are for different final resolutions in the range between $25 \mathrm{~nm}$ and $50 \mathrm{~nm}$. The strong increase of the possible frame number is only connected to the change of the intermediate resolution, proving the effectiveness of the DyMIN concept.

At the resolution which corresponds to the maximum of the curve in $(\mathrm{A})$, the bleaching of the sample is minimized. The fluorescence signal can be simultaneously maximized when integrating the fluorescence over a longer time period. Each peak in (A) appears approximately at the same resolution where a minimum of the simulated light dose is present in figure 5.5 (C). This suggests the numerical simulation is capturing the essential aspects of DyMIN. Figure 5.5 (B) shows the photobleaching of the fluorescence signal of fluorescent nanobeads with a nominal diameter of $40 \mathrm{~nm}$ (Crimson beads, Molecular Probes) vs. the number of frames acquired with MINFIELD, DyMIN, RESCue and conventional scanning. A confocal impression (for conventional scanning and DyMIN) of the sample after the bleaching experiments have been performed is shown in figure 5.5 (D). A more detailed data representation of $(\mathrm{C})$ is found in appendix A.3. The strong bleaching reduction by DyMIN compared to conventional scanning is evident.

The results should also be valid not only for single dye molecules [1, but for fluorophore clusters with a small diameter. All localized structures which have an unlabeled sample area between each other and are more or less small enough to fit into the MINFIELD condition (nuclear pore complexes, viruses, membranes, filaments, ect.), can benefit from DyMIN. However, if the structure size starts to exceed approximately $250 \mathrm{~nm}$, the advantage of DyMIN will slowly vanish. 

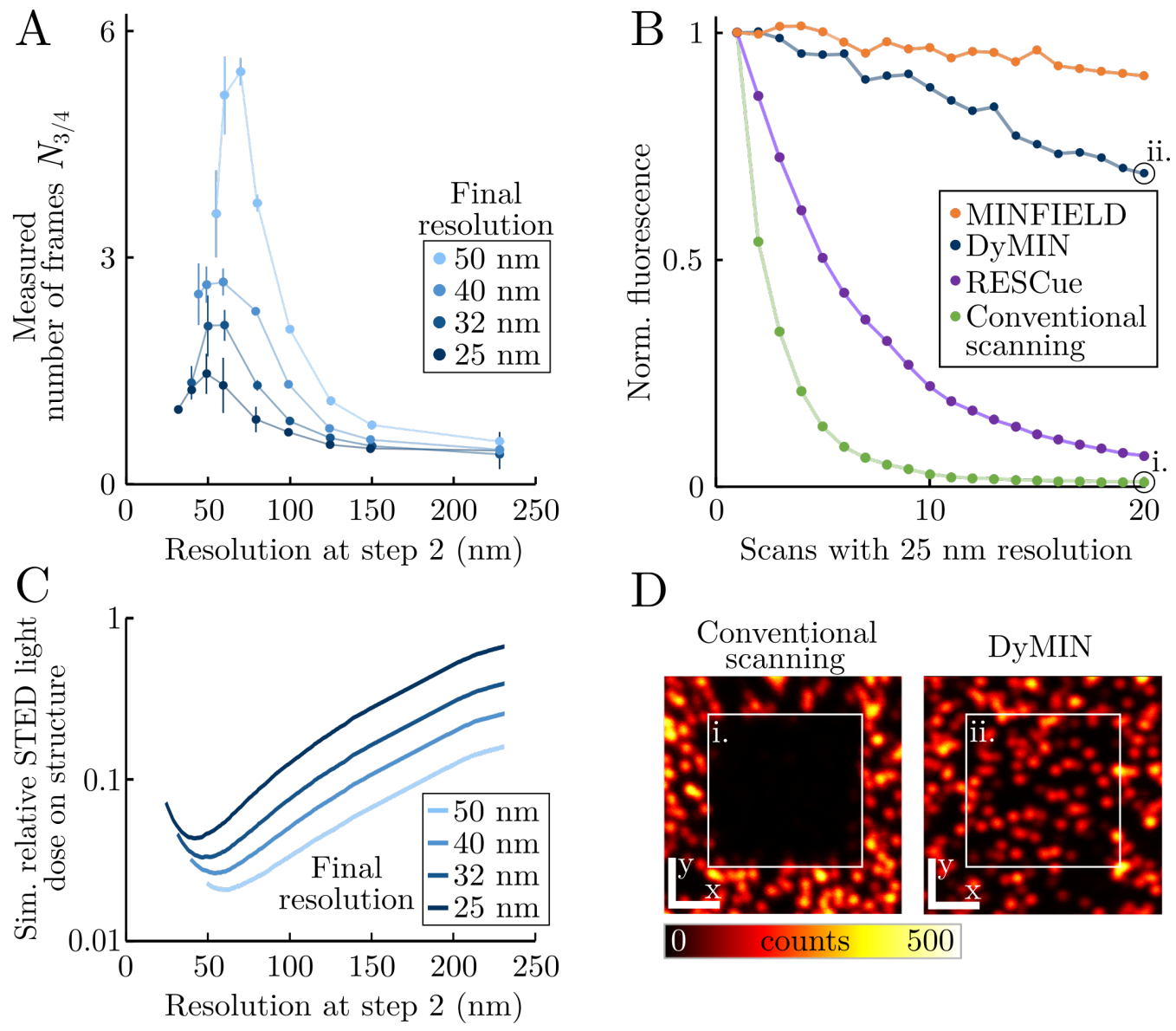

$\mathrm{D}$

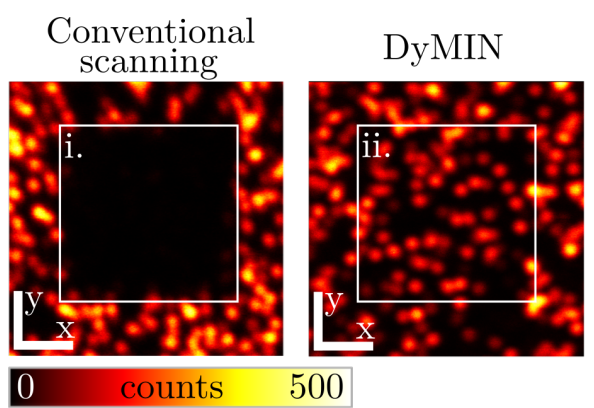

Figure 5.5.: Reduction of photobleaching by a 3-step DyMIN scan. The effectiveness of DyMIN scanning strongly depends on the resolution of the intermediate probing steps. (A) The intermediate resolution (step 2) significantly influences the number of frames $N_{3 / 4}$ which can be recorded before the fluorescence level drops to $75 \%$ of the initial value. Shown is $N_{3 / 4}$ vs. the intermediate resolution, measured for varying final resolutions between $25 \mathrm{~nm}$ and $50 \mathrm{~nm}$. Sample consisted of $\sim 23$-nm-sized fluorescent nanoassemblies (Bead R, GattaQuant), pixel size was $15 \mathrm{~nm}$ and the decision time for both probing steps (step $1+2) 10 \mu \mathrm{s}$. Each data point is the mean of 5 individual measurements with \pm 1 st. dev. The lines are a guide to the eyes. (B) Decrease of fluorescence level caused by photobleaching vs. number of acquired frames at a resolution of $25 \mathrm{~nm}$ for MINFIELD (150 nm field), a 3-step DyMIN scan, RESCue and conventional scanning. The fluorescence level is the average of the corresponding confocal step. Sample consisted of fluorescent nanobeads with a nominal diameter of $40 \mathrm{~nm}$ (Crimson). The MINFIELD curve is a median of ten individual single beads. (C) Simulated STED light dose on the structure for a sample remodeling the experiment in (A) as a function of the intermediate resolution in step 2. The bleaching minimum observed in (A) appears in the range of the light dose minimum found by the simulation. (D) Confocal impression of the bleached areas after the experiment of (B) has been performed, (i) conventional scanning (left) and (ii) DyMIN (right). Scale bars $(x, y)$ are $1.5 \mu \mathrm{m}$ (D). Figure reproduced from ref. 2 . 


\subsection{D-STED DyMIN applications}

In this section, 2D-STED DyMIN is experimentally evaluated on biological and technical samples. A STED light dose reduction on the sample structure up to $\sim 173$-fold is realized (4-step DyMIN), and a signal gain of $\sim 9$-fold for biological samples (3-step DyMIN).

\subsubsection{Applying DyMIN to biological samples}

The effectiveness of a 3-step DyMIN method is evaluated for biological samples with different structure types and varying sample density. First the DyMIN benefit is investigated on the known periodic structure of betaII spectrin which is formed orthogonal to the distal axon direction in neurons (Figure 5.6 (A)). This structure was first revealed by STORM microscopy [110]. The distance between individual betaII spectrin structures is closer $(192 \mathrm{~nm})$ than the diffraction limited resolution 111]. For the structure shown, DyMIN increased the fluorescence signal which was extractable from the sample by a factor of 3 compared to conventional scanning. For both scanning concepts, the dwell time was adjusted accordingly for the maximum signal, see section 2.3 . An even higher signal increase is observed for small-sized synapses 112 , see figure $5.6(\mathrm{~B})$ ).

Here in particular, a gephyrin scaffolding protein of inhibitory synapses is shown. It can be organized as a mono- or multicluster 113 115. Gephyrin is imaged with 3-step DyMIN which localizes the clusters during the scan on the fly with low (confocal) resolution $(230 \mathrm{~nm}$ ) and a resolution at low STED power (roughly $72 \mathrm{~nm}$ ). Only at those sample positions where the localized clusters are present, the final STED power corresponding to a resolution of $\sim 24 \mathrm{~nm}$ (see appendix A.1) is applied. The pixel dwell time in the DyMIN scan (Figure 5.6 (B), right) was set to $100 \mu \mathrm{s}$, which maximizes the fluorescence signal (compare section 2.3). Such a long dwell time is only useful because of the lower bleaching provided by DyMIN. For conventional scanning the dwell time had to be lowered to $10 \mu \mathrm{s}$ in order to achieve the maximum signal of the sample at the same resolution as for DyMIN (Figure 5.6 (B), left). Otherwise the high light dose (far behind the signal optimum) was inducing too much bleaching. With conventional scanning at the resolution used, the maximum fluorescence signal is too low to define the gephyrin structure (Figure 5.6 (B), left). With DyMIN it was possible to record the gephyrin clusters with a superior fluorescence signal (Figure 5.6 (B), right). According to equation 2.5, the 9 times higher signal achieved by DyMIN could have been transformed into a resolution which is $\sim 42 \%$ better than without DyMIN at an identical fluorescence signal. 

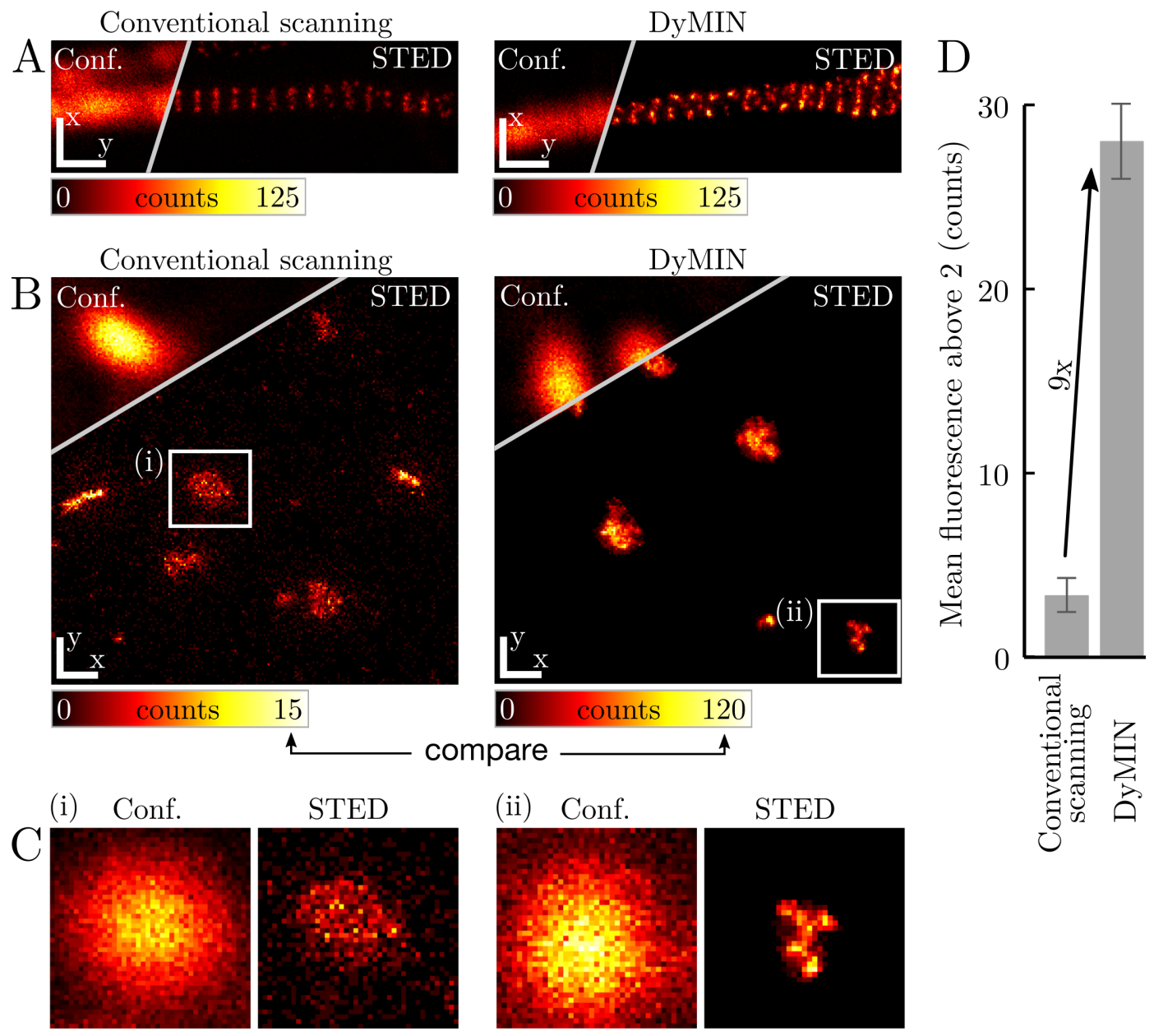

Figure 5.6.: DyMIN increases the image brightness and structural definition. At a certain high resolution, any STED recording is hampered by photobleaching. The lower light dose by DyMIN results in lower bleaching which can be used to increase the fluorescence signal for a better structure definition or to push the final resolution. (A) Shows the periodic betaII spectrin cytoskeleton which forms along the axons in hippocampal neurons of rats, resolved with conventional STED scanning (left) and with DyMIN scanning (right), both with a final resolution of $\sim 29 \mathrm{~nm}$. The DyMIN scan used a longer dwell time $(100 \mu \mathrm{s})$ compared to the conventional scan $(40 \mu \mathrm{s})$, to make use of the lower bleaching (higher signal), see section 2.3. (B) Gephyrin clusters of the inhibitory synapses of rat hippocampal neurons. With DyMIN, a $\sim 9$-fold higher fluorescence signal was obtained compared to the conventional scanning at a final resolution of $\sim 24 \mathrm{~nm}$, the dwell time was $100 \mu \mathrm{s}$ and $10 \mu \mathrm{s}$, respectively. (C) Confocal and STED image details of (B) for conventional scanning (i) and DyMIN (ii). DyMIN allows to observe the nanoscale morphology at superior signal to noise. (D) The quantitative signal gain for measurements as presented in (B). More than 100 different clusters from 6 independent measurements each were analyzed for DyMIN and conventional scanning (data is the mean value with \pm 1 st. dev. of the mean signals). Scale bars $(x, y)$ are $500 \mathrm{~nm}(\mathrm{~A})$ and $200 \mathrm{~nm}$ (B). Figure reproduced from ref. [2]. 


\section{Adaptive-Illumination STED Nanoscopy}

The reduction of the STED light which is applied to the imaged structure, as well as the light dose reduction for the complete imaged scan field, can be calculated with the simulation frame work. The illumination masks which form during the DyMIN scans carry the entire information about the applied illumination steps in each pixel. The illumination masks from the experiments were saved after the DyMIN acquisition. When combining these masks with the STED powers as well as corresponding decision and dwell times, the total spatial dose can be computed. To calculate the light dose acting on the structure, all high-pixels of the final DyMIN step were defined as the structure. Specifically, a 3-fold lower STED dose which acts directly on the structure was determined for the structure in figure 5.6 (A). A $\sim 13$-fold lower dose directly on the clusters was inferred for the gephyrin recording in figure 5.6 (B), and $\mathrm{a} \sim 25$ times lower overall light dose was achieved. This is an important factor for live-cell experiments. A lower overall light dose may help to lower the phototoxicity 116 117. The simulated light dose retrenchments on the structure by DyMIN are in good agreement with the fluorescence signal gains observed in the experiment. A $\sim 3$ times higher signal was recorded for betaII spectrin, and $\mathrm{a} \sim 9$-fold brighter image for gephyrin (see figure 5.6 (D)). All imaging parameters used in this thesis are listed in the appendix A.2.

\subsubsection{Evaluation of the 2D-STED DyMIN resolution}

For fluorophore clusters of a size of about $<100 \mathrm{~nm}, 4$-step DyMIN has conceptual benefits. A larger area surrounding the structure can be probed to be empty in a light-efficient way, thereby the minimum is better centered above the structure for the final high-resolution step. The structure experiences a much lower STED light dose which is demonstrated on dye-labeled DNA origami structures 118, providing an outstanding contrast at a resolution (FWHM) of approximately $17 \mathrm{~nm}$ (Figure 5.7).

The used origami structure features two fluorophore groups with $\sim 15$ ATTO $647 \mathrm{~N}$ dye molecules for each group. The spots have a nominal distance of $30 \mathrm{~nm}$ from center-to-center. Due to the spot extent along the origami structure, a gap of only $10 \mathrm{~nm}$ is present (Figure 5.7 (C)). The 4-step DyMIN scan was able to lower the light dose on the structure by $\sim 173$-fold compared to the conventional scan, as found by the simulation of the scan after it was completed.

A relatively long dwell time of $150 \mu \mathrm{s}$ was chosen which maximized the signal in the image. Attempts to image the origami nanostructure without the use of DyMIN failed due to the much higher bleaching in the conventional STED recording. Again, the dwell time in the conventional scan was lowered in order to achieve the signal optimum. However, even at a dwell time reduction of 300 -fold $\left(t_{i m g}=500 \mathrm{ps}\right)$, the 
A

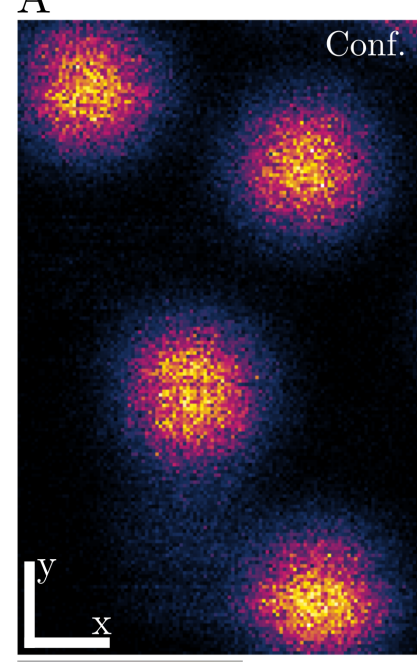

0 counts 57
$\mathrm{B}$

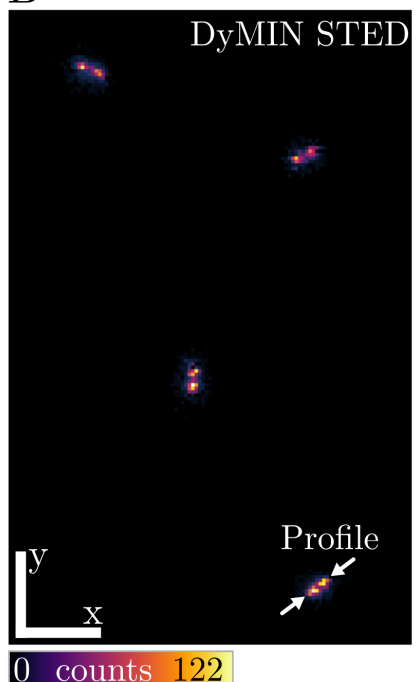

C
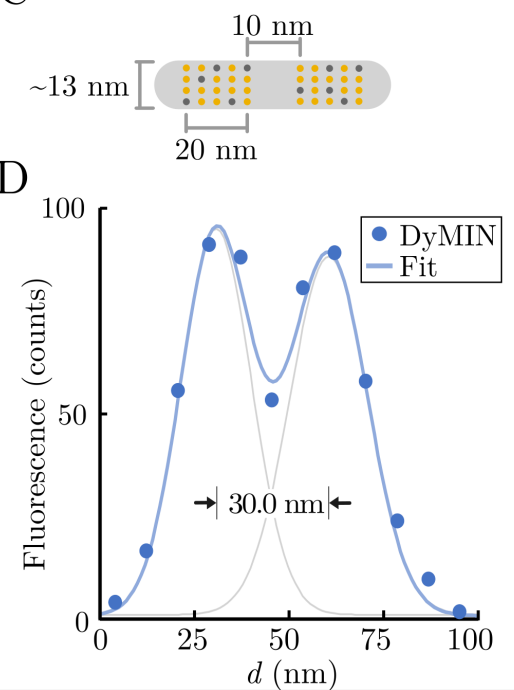

Figure 5.7.: DyMIN recording with high resolution and fluorescence signal. Sample structure is a DNA origami-based nanoruler (GATTAquant) with two fluorophore assemblies which are nominally separated by $30 \mathrm{~nm}$, center-to-center. (A) Confocal step image, and (B) DyMIN recording. The two spots resolved by DyMIN consist on average of $\sim 15$ fluorophores (ATTO 647N) each. (C) Scheme of the nanoruler assembly. Fluorophores (yellow) bind randomly on empty coupling points (gray) with a chance of $\sim 75 \%$ (Nanoruler shape and fluorophore binding efficiency according to manufacturer). (D) Plot of the intensity data of the profile highlighted in (B). The fit is a double Gaussian with FWHMs of $22.1 \mathrm{~nm}$ and $24.2 \mathrm{~nm}$. The peak-to-peak separation is measured to be $30.0 \mathrm{~nm}$. Taking the known extent $(\sim 20 \mathrm{~nm}$, remark $(\mathrm{C}))$ of the fluorophore groups into account, the real width of the effective PSF needs to be around $17 \mathrm{~nm}$ (FWHM). This maximum system resolution (at $40 \mathrm{MHz}$ ) is in perfect agreement with appendix A.1. Scale bars $(x, y)$ are $200 \mathrm{~nm}$ $(\mathrm{A}, \mathrm{B})$. Figure reproduced from ref. [2].

bleaching dose was much higher as it should be for the signal optimum. This was confirmed by the fluorescence level after the acquisition, which was significantly lower than $1 / e^{2}$ of its original value. This observation indicates a non-linear bleaching effect at this very high STED intensity (average STED power of $450 \mathrm{~mW}$ in the focus; the SLM was passed only once). 


\subsection{D-STED DyMIN}

The DyMIN concept is not limited to 2D-STED only. It is also capable to reduce photobleaching for 3D-STED when increasing the resolution in $z$ (or in each direction using the combination of 2D- and 3D-STED). In this section, the best intermediate $z$-resolution for a 3-step DyMIN method is investigated. Furthermore, a volume is imaged with DyMIN at an isotropic resolution of approximately $70 \mathrm{~nm}$ with high fluorescence signal.

\subsubsection{Experimental parameter optimization}

To find the best intermediate resolution, dispersed fluorescent nanobeads are imaged in an $x z$-scan with 3 -step DyMIN. The intermediate resolution was varied and the resulting photobleaching observed. In figure 5.8 the number of frames $N_{3 / 4}$ vs. the intermediate axial-STED resolution is shown (final resolution is $75 \mathrm{~nm}$ ). Likewise as for 2D-STED DyMIN, an intermediate resolution exists which minimizes the photobleaching (maximum in the curve). For the parameters used here, the best step 2 resolution appears close to half the final resolution.

A

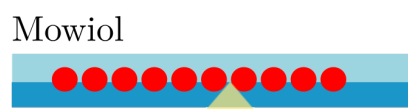

Coverslip

\section{Objective}

B

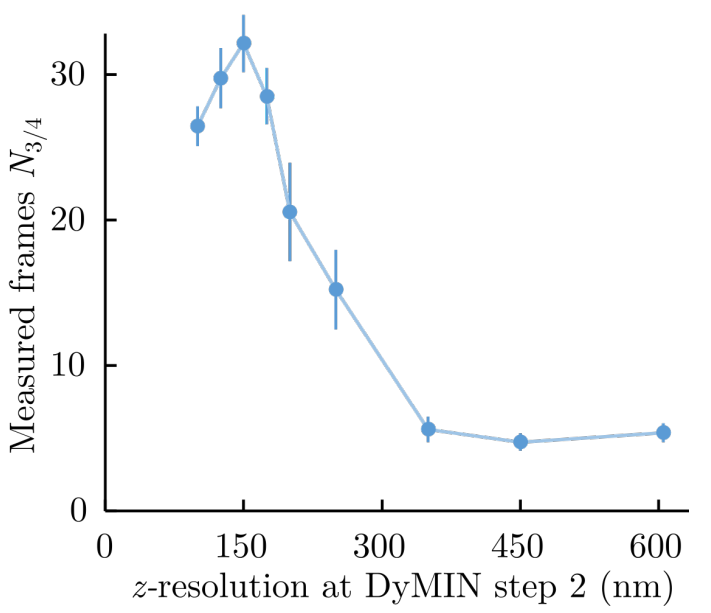

Figure 5.8.: Bleaching reduction for 3D-STED with 3-step DyMIN. (A) Sample: a quasi mono-layer of $\sim 40 \mathrm{~nm}$-sized fluorescent nanobeads (Crimson, Molecular Probes) mounted on a coverslip. (B) Shown is the number of frames $N_{3 / 4}$ (blue dots) after the fluorescence level is decreased to $75 \%$ of the initial value vs. the $z$-resolution of the intermediate probing step, using axial-STED. The blue line is a guide to the eyes. Pixel size $(x, z)$ was $30 \mathrm{~nm}$, fixed decision times of $20 \mu \mathrm{s}$ for both probing steps. Figure reproduced from ref. [2]. 


\subsubsection{Improved imaging of a volume}

As for all volume acquisitions with 3D-STED, the maximum resolution and signal is limited by photobleaching. While imaging a plane of a fixed sample with 2DSTED only once, each plane of a volume is imaged (and is thereby illuminated / bleached) several times. DyMIN was used to image a volume with high 3D-STED resolution and with a good signal-to-noise ratio. The bleaching reduction by DyMIN is investigated on mature mouse spermatozoa. The results are shown in figure 5.9 . A confocal overview of the sperm arrangement is presented in figure 5.9 (A).

For conventional scanning, the size of the volume needs to be set in a way that it includes the complete structure of interest. Typically biological structures often have a complicated shape surrounded by empty space or even include such. When choosing conventional scan regions for imaging, those will contain empty spaces leading to needless bleaching by imaging of those areas. Setting the imaging boundaries close to the structure, which is already possible with a simple 2-step DyMIN method (confocal probing followed by the finale image), avoids a lot of illumination on the structure. Nevertheless, for small structures 3-step DyMIN can reduce the bleaching further by setting the boundaries even closer to the structure of interest while centering those in the axial-STED OFF-switching minimum.

In the fixed sample, the microtubulin in spermatozoa is immunofluorescence-labeled. In the axonems, tubulin fibers are known to form a tube-like structure which has a diameter of $\sim 100 \mathrm{~nm}$, consisting of nine microtubule doublets arranged like a ring and another tubulin doublet in the center of the tube (cross section view). A volume of the sperm sample was imaged with 3D-STED, see figure 5.9 (B). The power between the 2D-STED PSF and axial-STED PSF was adjusted for an isotropic resolution. The $x$ - and $z$-axis were used as the fast axes, and the $y$ axis as the slow axis ( $x z y$-scan). Imaging was performed with (i) conventional scanning and with (ii) DyMIN. For (i) the dwell time was lower compared to (ii) by a factor of 13.5, which results for both scanning approaches in the best maximum signal at this resolution and pixel size. Trying to image in the conventional mode with the same dwell time $(80 \mu \mathrm{s})$ as for DyMIN, resulted in such high bleaching, that no structure was observable. However, at the signal-optimized dwell time for conventional scanning, at least some (but not enough for structure definition) fluorescence was observed. A normal approach in this case is to reduce the (STED) light dose, but as a consequence, the tubulin structure would not be resolved by the lower resolution.

DyMIN enables to image a complete stack with sufficient signal at a resolution for a clear structure definition. The tube-like arrangement is visible in the raw data, allowing a three-dimensional rendering of the sperms as shown in figure 5.9 (C). With DyMIN, a $\sim 22$-fold light dose reduction for the complete stack was realized compared to the conventional counterpart. Analyzing the fluorescence signal shows a $\sim 14$-fold brighter image in contrast to the conventional scan. 


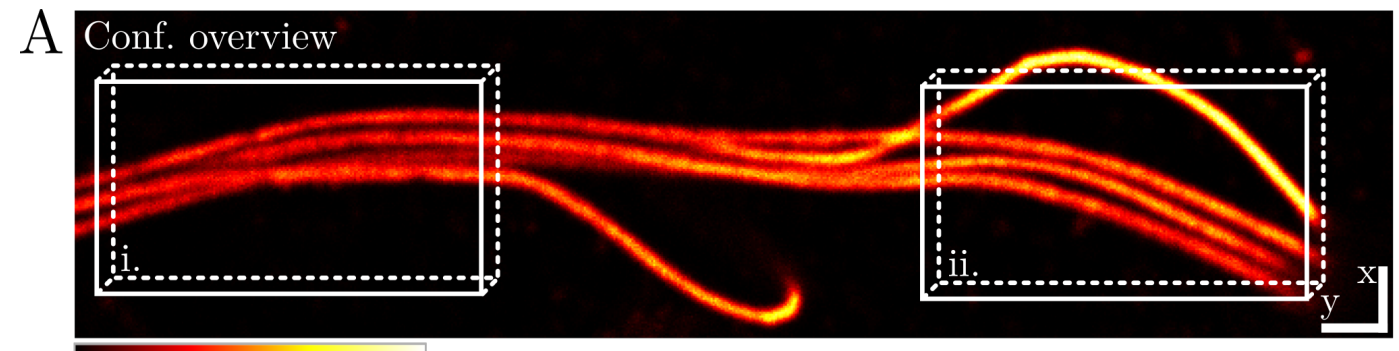

0 counts 340

i. Stack conventional scanning

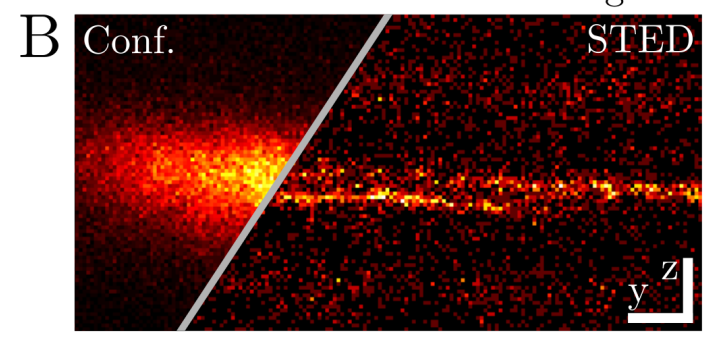

ii. $\quad$ Stack DyMIN
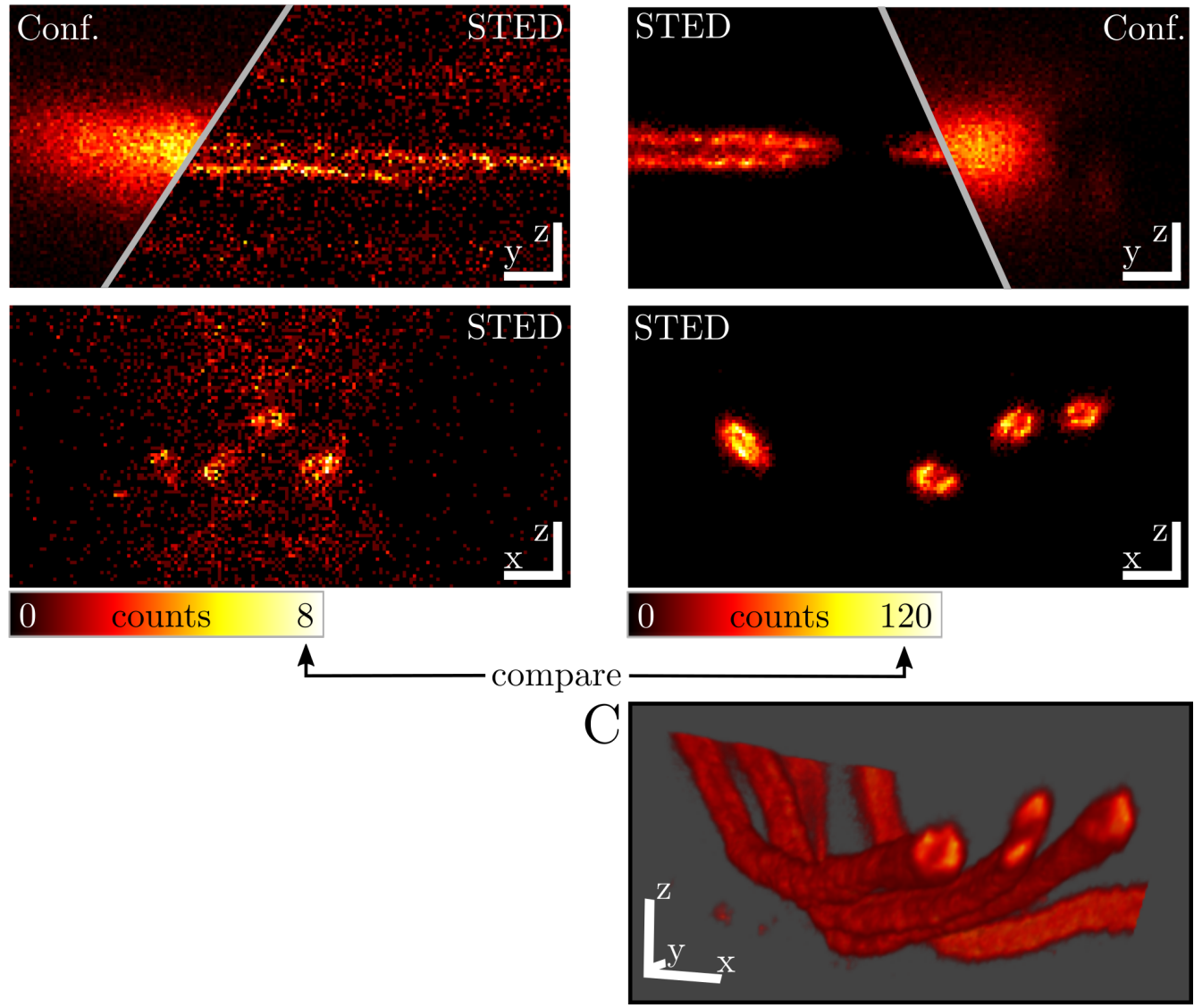

Figure 5.9.: Lower bleaching by DyMIN enables a volume acquisition with isotropic resolution of tubulin structure in mouse spermatozoa. Sample: tubulin in the axonemes of mouse spermatozoa (indirect immunofluorescence, see section 7.2). (A) Confocal impression. (B) Different views ( $y z$ - and $x z$ - slices taken from the xzy volume-recording) through the tube-like structure with conventional scanning (i) and DyMIN (ii), both with an isotropic resolution of $\sim 70 \mathrm{~nm}$. The voxel cube length is $30 \mathrm{~nm}$. Dwell time is $6 \mu \mathrm{s}$ for conventional scanning, $80 \mu \mathrm{s}$ for DyMIN, which is at the signal optimum for both. The fluorescence signal with DyMIN is 14-fold higher (compare lookup tables in (B)) and enables the reliable identification of structure isosurfaces $(\mathbf{C})$ for a volume rendering of the tubulin. Scale bars: $3 \mu \mathrm{m}(x, y)(\mathrm{A}), 500 \mathrm{~nm}(x, z$ and $y, z)(\mathrm{B})$. Coordinate bars in $(\mathrm{C})$ are $1 \mu \mathrm{m} \times 1 \mu \mathrm{m} \times 1 \mu \mathrm{m}$, the $y$-axis points towards the reader. Figure reproduced from ref. $[2]$. 


\subsubsection{Evaluation of the 3D-STED DyMIN resolution}

A 4-step DyMIN scan was applied to image DNA origami structures with 3D-STED. The origami structure is a nanopillar, standing at the coverslip surface (Figure 5.10 (A)). Two fluorophore groups are seperated by nominally $70 \pm 5 \mathrm{~nm}$, and each one consists of $\sim 11$ ATTO $647 \mathrm{~N}$ dye molecules which connect randomly to 15 available binding spots at each group. The size of both groups is different; one has an axial extent of nominally $15 \mathrm{~nm}$ and the other one of $25 \mathrm{~nm}$. In figure 5.10 (B) a confocal recording (left) of the nanopillar is shown in an $x z$-view. The 3D-STED recording (right) clearly resolves both fluorophore groups. Relative power between the 2D and axial OFF-switching pattern was $15 \%$ and $85 \%$, respectively (slightly better resolution in the axial direction [57]). The maximum STED pulse power at the typical system frequency of $40 \mathrm{MHz}$ was too low to achieve the necessary resolution for a clear separation. In order to attain a doubled pulse power $(\sim 22 \mathrm{~nJ})$, the microscope was tuned to $20 \mathrm{MHz}$ (the STED laser pulse shape was stretched to $\sim 940 \mathrm{ps}$ to achieve a good conversation efficiency in the SHG crystal). The pulse energy used for the measurement would at $40 \mathrm{MHz}$ result in a STED power in the sample of $\sim 688 \mathrm{~mW}$.

A

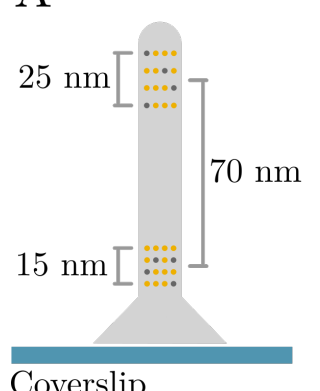

B

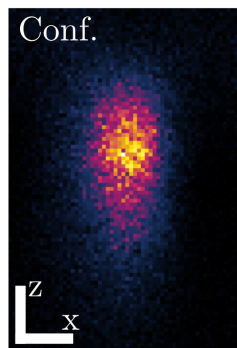

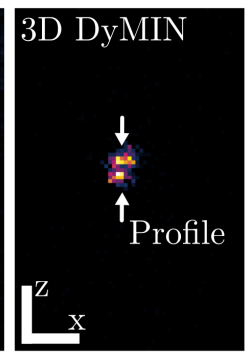

$\mathrm{C}$

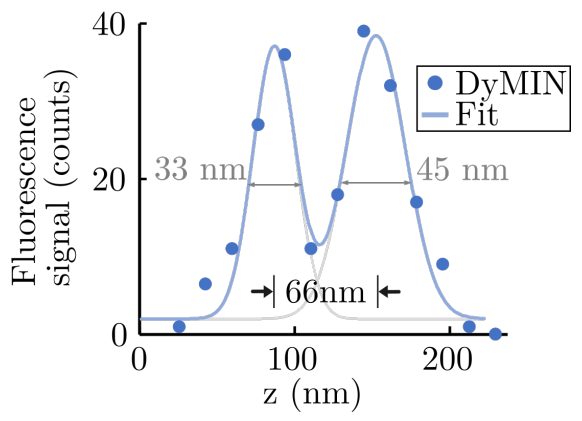

Figure 5.10.: DyMIN recording with very high 3D-STED resolution. Sample structure is a DNA origami-based nanopillar (GATTAquant) with two fluorophore assemblies with a nominal distance of $70 \mathrm{~nm} \pm 5 \mathrm{~nm}$. (A) Schema of the nanopillar assembly. The fluorophores (yellow) bind randomly on empty coupling points (gray) with a chance of $\sim 75 \%$ (Nanopillar shape and fluorophore binding efficiency according to manufacturer). (B) Confocal (left) and 4-step DyMIN recording (right) of a nanopillar. The two resolved spots consist of $\sim 11$ fluorophores on average. (C) Plot of the measured intensity data vs. the axial position. Fitted is a double Gaussian with a FWHM of $33 \mathrm{~nm}$ and $45 \mathrm{~nm}$, featuring a clear separation of both spots. Scale bars $(x, z)$ are $200 \mathrm{~nm}(\mathrm{~B})$. 


\section{Adaptive-Illumination STED Nanoscopy}

Using equation 1.4. taking into account the confocal resolution enhancement for a pinhole size of $0.75 \mathrm{AU}\left[13\right.$, and the measured $P_{S}$ for this dye of $\approx 2.464 \mathrm{~mW}$, the theoretical $z$-resolution for these parameters is $\sim 34 \mathrm{~nm}$. When approximating the effective fluorescence spot and the fluorophore group geometry as a Gaussian, the following formula can be used to approximate the theoretical appearing structure FWHM $\left(S_{F W H M}\right)$ in the image

$$
S_{F W H M}=\sqrt{O_{s i z e}^{2}+d_{m i n, z}^{2}},
$$

with the real structure size $O_{\text {size }}$. For the small $(15 \mathrm{~nm})$ fluorophore group an $S_{F W H M}$ of $37 \mathrm{~nm}$ results, and for the larger $(25 \mathrm{~nm})$ group an $S_{F W H M}$ of $42 \mathrm{~nm}$. Both values are close to the measured values, indicating an effective PSF (FWHM) along $z$ of remarkable $\sim 34 \mathrm{~nm}$.

\subsection{Multi-color DyMIN imaging}

The use of DyMIN for multi-color recordings is straightforward using dyes with different absorption/emission wavelengths in combination with different excitation wavelengths and detection windows. The best imaging results can be achieved when each dye is imaged (illuminated) with its own optimized settings (especially the STED power) 79. In figure 5.11 NPCs (located in the nuclear envelope) and lamina (located on the inside of the nuclear envelope) are presented. Both were imaged with 3D-STED DyMIN, featuring an isotropic resolution.

A resolution of roughly $\sim 60 \mathrm{~nm}$ (structure FWHM of $\sim 73 \mathrm{~nm}$ ) was observed for the NPCs, and a somewhat lower resolution for the lamina structure (the $I_{s}$ for the lamina staining is higher). The antibody which is used for NPC labeling, is known to label different proteins of the NPC complex (Section 7.2). With DyMIN, it was possible to clearly resolve the two labeled spots of the NPC which form close to the nuclear basket and to the membrane ring and central channel, with superior resolution and fluorescence signal. Lamin is found to be approximately in the center between both spots.

A further bleaching reduction was realized by imaging the redder dye (NPCs) first, and the greener dye (lamin) afterwards (frame interleaved). Otherwise (quasi simultaneous acquisition) the red dye experiences some useless state cycling (which is typically higher for the green dye compared to the red dye for the same resolution 79]) by the green excitation in combination with the STED illumination, and is thereby bleached without any use. The green dye, however, is almost not excited by the red excitation and almost no bleaching of the green dye occurs during the acquisition of the red dye. 


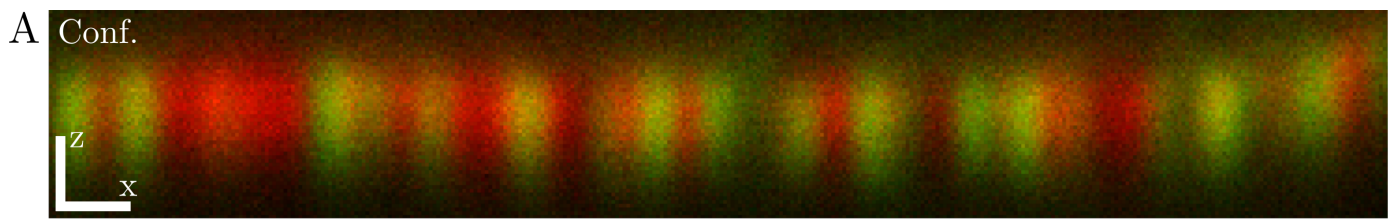

0 counts $151 \quad 0$ counts 245

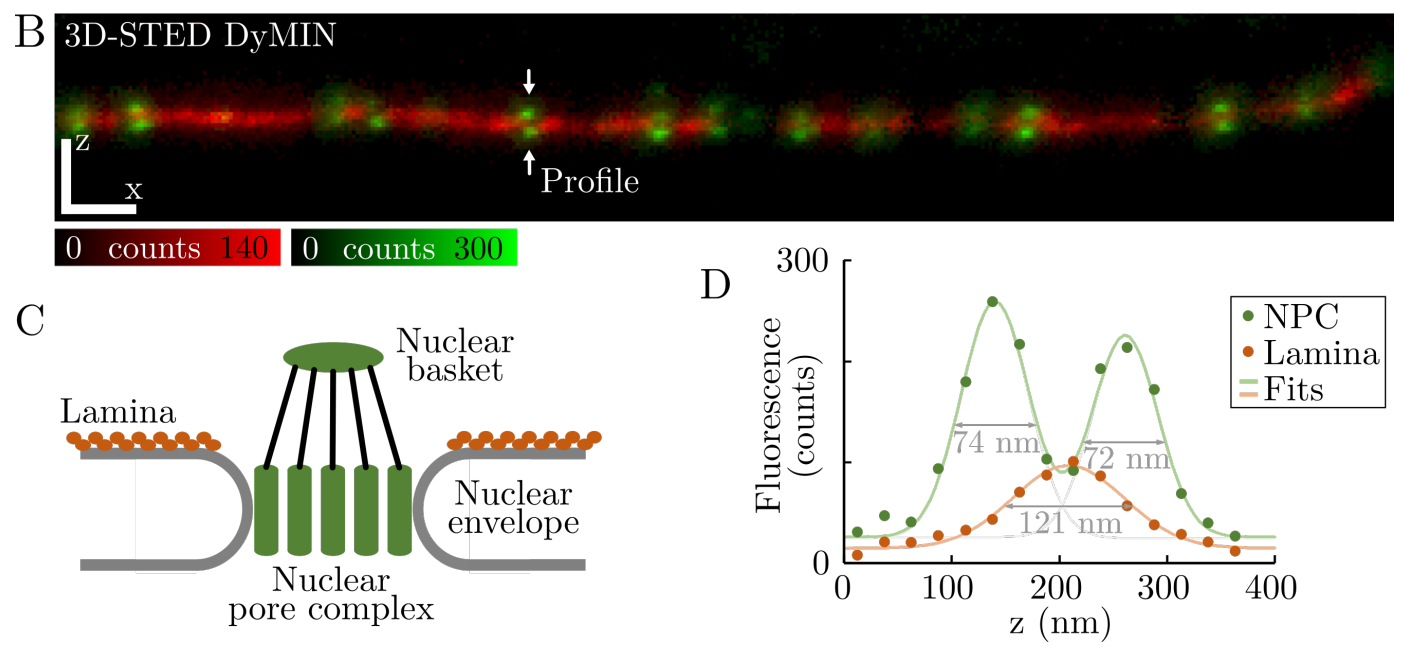

Figure 5.11.: Two-color DyMIN imaging of NPCs and lamina. (A) Recording of the confocal step. (B) 3D-STED recording with an isotropic resolution, using 3-step DyMIN. NPCs are shown in green and the lamina in red. With DyMIN, two spots are clearly resolved which are formed by the nuclear basket and the central channel + membrane ring. For both color channels the pixel size is $25 \mathrm{~nm} \times 25 \mathrm{~nm}$, and the dwell time $80 \mu \mathrm{s}$. (C) Scheme of the spatial arrangement of the labeled structure in $(\mathrm{A}, \mathrm{B})$. (D) A plot of the data taken along $z$, as indicated in (B). The green line represents a fit with a double peak Gaussian for the NPC, the red line shows a single Gaussian fit (data is the average signal of 10 pixels along the $x$ direction) of the lamina. Scale bars $(x, z)$ are $500 \mathrm{~nm}$ for $(\mathrm{A}, \mathrm{B})$. Figure reproduced from ref. [2]. 


\subsection{Heterogeneous photobleaching and structure probing}

The current pixel-wise DyMIN implementation has two weaknesses: One is the uneven bleaching which occurs when approaching big structures orthogonally to the slow scan axis (Figure $5.12(\mathrm{~A})$ ). The second is the low acceptance for large signal variations with fixed thresholds, which results in wrong decisions whether to continue imaging at any step $i$ or stop imaging in some pixels, see figure 5.12 (B). Uneven bleaching can be strongly reduced by scanning the sample area several times (with a fraction of the final dwell time), and summing the fluorescence signal of each scan (similar to the MINFIELD scan approach). To do so, the final illumination mask needs to be stored and repeatedly used for scanning at the final step $N$ to avoid useless reprobing of the structure.

The variation of the excepted signal can be enlarged in a related way. At each DyMIN power $P_{i}$, the complete sample area can be imaged, collecting all information at the respective resolution. Since the Nyquist criterion is fulfilled, each pixel contains at least some information of all eight surrounding pixel. If now statistically one pixel was aborted too early, or a single pixel is kept on without neighboring pixel being on, the individual decision can be reversed by the provided knowledge of all pixels. Furthermore, a more intelligent algorithm could recognize such partly probed sample areas as in figure 5.12 (B) and either fill the final illumination mask or remove it completely at this positions. 

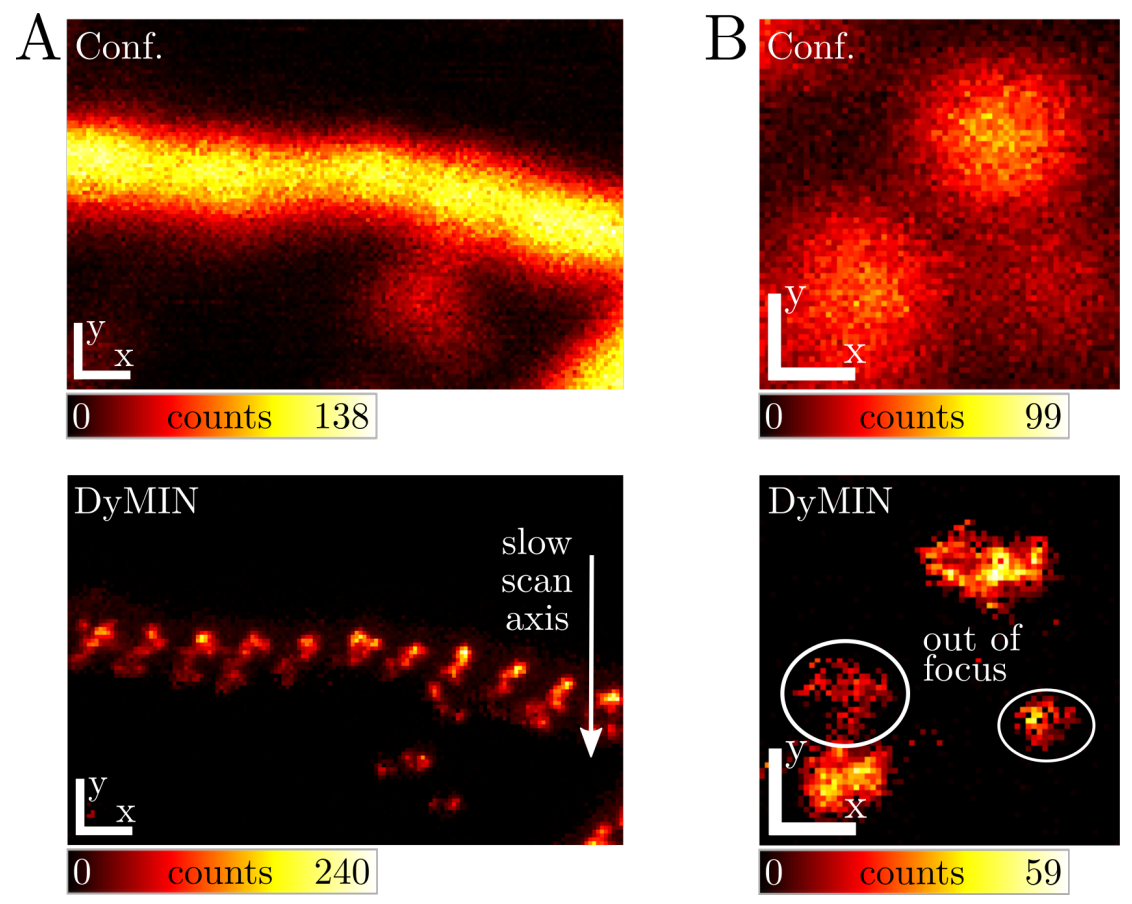

Figure 5.12.: Weaknesses of the here presented pixel-step DyMIN implementation. (A) Shown is a confocal (top) and 3-step DyMIN (bottom) recording of the periodic betaII spectrin structure (compare figure 5.6 (A)) which is roughly orthogonally oriented according to the slow DyMIN scan axis. In this first DyMIN implementation, this way of approaching an extended structure leads to uneven bleaching. Here, concretly, the upper part of the structure appears brighter (less bleached before imaged) compared to the lower part. (B) Confocal (top) and 3-step DyMIN image (bottom) of gephyrin (compare figure 5.6 (B)). In this first DyMIN implementation, fixed thresholds are used which are not able to capture large variations in the fluorescence signal (originating for instance from defocus). As a result, some pixels are not imaged (the DyMIN illumination list was aborted early), compare encircled areas in (B, bottom). Scale bars $(x, y)$ are $200 \mathrm{~nm}(\mathrm{~A}, \mathrm{~B})$. Figure reproduced from ref. [2]. 



\section{Conclusion and Outlook}

\subsection{Summary}

The connection between the maximum fluorescence signal which can be collected from a sample and the attainable STED resolution was derived (Chapter 2). For every sample, a certain fluorescence photon budget exists, limiting the maximum possible STED resolution and fluorescence signal. It was found that increasing the STED resolution causes photobleaching to increase accordingly to the power of four. As a reverse conclusion, fluorescence signal can be converted into a higher STED resolution. Hence, optimizing the fluorescence photon budget will always facilitate the highest STED resolution possible. To achieve higher fluorescence signals, several methods have been developed.

The quality of the STED OFF-switching minimum has a direct impact on the fluorescence signal. A microscope was built, which uses an SLM to create and optimize the phase masks for 2D- and axial-STED and to arrive at high-quality OFF-switching minimums (Chapter 3). Optical aberrations have been corrected under different challenging imaging conditions to provide a high fluorescence signal. Furthermore, the SLM enabled to use several objective lenses with axial-STED without any microscope adaptations, which is not possible with conventional phase mask elements. With the correctly chosen objective lens, live-cell 3D-STED was performed without a decrease of the fluorescence signal and with good 3D-resolution up to a depth of $\sim 37 \mu \mathrm{m}$.

With the new MINFIELD illumination scheme, a much higher fluorescence signal and STED resolution is possible (Chapter 4). MINFIELD was performed with galvanometric scanners, which allows a much simpler microscopy setup, featuring a bigger application range for imaging compared to the original optical setup [1]. MINFIELD was extended to 3D-STED, whereby a superior isotropic resolution of $\sim 60 \mathrm{~nm}$ was achieved.

In this thesis, the novel illumination concept of a dynamic intensity minimum (DyMIN) was developed. With DyMIN, it is possible to dynamically probe for structures and set the imaging boundaries for an arbitrary shape close to the structure while scanning a large field of view (Chapter 5). Thereby bleaching is significantly reduced, which results in a higher fluorescence photon budget. With 


\section{Conclusion and Outlook}

DyMIN, a $\sim 20$-fold lower light dose was achieved under biological imaging conditions and a more than 100-fold light dose reduction for sparser samples. The improved fluorescence signal provided by DyMIN was converted into the highest achieved 2D- and 3D-STED resolutions (FWHM) with a single objective lens for a large field of view, $\sim 17 \mathrm{~nm}$ in the lateral plane (Figure 5.7 ), and $\sim 34 \mathrm{~nm}$ in the axial direction (Figure 5.10).

\subsection{Improving conventional STED scanning}

In STED fluorescence nanoscopy, sample structures are labeled with fluorescent dyes. The dyes are switched between a fluorescent ON-state and a non-fluorescent OFF-state. During each ON/OFF-transition, a finite probability exists that a dye molecule is (photo-)bleached, which means it cannot contribute to the sample structure definition. At high levels of photobleaching, resulting from an intense illumination which is needed for high STED resolutions, the sample fluorescence signal may be so low that an interpretation of the recorded structure is not viable. Several imaging parameters affect photobleaching or reduce the fluorescence signal during the STED scan. An optimization of each will facilitate the highest fluorescence signal and STED resolution.

The fluorescence signal in STED nanoscopy is affected by the quality of the OFFswitching minimum, the microscope alignment, and the amount of fluorescence photons that can be collected from a dye molecule before it transitions into an irreversible dark state. The quality of the OFF-switching minimum and alignment can arrive at an optimum. The same is true for the photostability of a fluorophore (no photobleaching at all). However, in practice such a fluorophore does not exist. For any densely labeled sample, a certain fluorescence integration time (dwell time) exists which features the maximum fluorescence signal collectable from a sample for a set of imaging parameters, as shown in the publication of DyMIN [2. At shorter or longer dwell times, in respect to the best dwell time, the fluorescence signal and structure definition in the image is lower than it could be (Figure 2.3). To derive the best dwell time, the approximation that bleaching is only induced by STED photons in a linear dependence is used [1]. The best dwell time depends on a bleaching constant of the specific fluorophore, an oversampling factor, and the STED resolution (Equation 2.3.

Because the STED resolution follows a square-root dependence on the applied STED power, a resolution increase results in an accordingly quadratic power- and light dose increase. Additionally, a higher resolution also requires a smaller pixel size to fulfill the Nyquist sampling requirements, meaning that at higher resolutions more pixels are scanned leading to a higher light dose. Taking both effects into 
account, the light dose applied to the sample increases mainly in dependence on the resolution increase $(\rho)$ to the power of four (Dose $\propto \rho^{4}$ ).

The maximum signal which can be collected is approximately proportional to $1 / \rho^{4}$ (Equation 2.5). Thus, a certain fluorescence signal can be converted into a higher STED resolution. For instance, a bleaching reduction by 10 -fold can be translated into a 10 times higher fluorescence signal or a $\sim 45 \%$ higher resolution (Figure 2.2. Preference may be given to the image signal or resolution, but to attain a maximum of both at the same time is impossible due to the limited, shared budget of fluorescence photons.

It becomes apparent that good STED images require proper setting of the pixel size and the oversampling factor to arrive at low bleaching light doses and short acquisition times. Also the dwell time is crucial, which should be set close to the best dwell time to gain the maximum fluorescence signal and hence structure definition. The best dwell time is found when the fluorescence level after the image acquisition is reduced to $1 / e^{2}$ of the initial level.

During this thesis, a STED microscope was built which uses an SLM to create the phase mask information which are necessary for the STED OFF-switching patterns. The optical SLM beam path design allows to imprint independent phase masks onto both of the orthogonal polarization directions of the STED beam without splitting the beam (Figure 3.1). The resulting 2D- and axial-STED OFF-switching patterns are superimposed in the focal spot for 3D-STED, enabling a resolution tuning in each direction.

It was demonstrated that the presence of astigmatism fills the minimum of the 2D-STED doughnut (Figure 3.2). This aberration was corrected with the SLM to achieve a nearly round OFF-switching pattern. The quality of the doughnut minimum was best with corrected astigmatism. A moderate astigmatism of \pm 0.15 resulted in a fluorescence signal drop of $\sim 20 \%$ at a STED power of $115 \mathrm{~mW}$. This finding is in good agreement with earlier theoretical results [95]. Another advantage of an astigmatism-corrected 2D-STED doughnut is the symmetric shape of the OFF-switching minimum, leading to an also symmetric resolution. In contrast with astigmatism present, the effective fluorescence spot would be elliptically shaped, featuring a non-isotropic resolution in the lateral plane.

The axial-STED OFF-switching pattern was also created with the SLM. It was shown that the diameter of the circular phase boundary of the phase mask has a significant impact on the OFF-switching pattern shape, steepness of the minimum, and resulting fluorescence signal. With a strongly mismatched circle diameter no useful minimum for axial-STED was observed (Figure 3.3). Such a mismatch results when the objective lens is changed to a different kind (e.g. from oil-immersion to water-immersion), because this changes the size of the objective lens back aperture. With the SLM, the circle diameter can easily be adjusted to the objective lens. As 


\section{Conclusion and Outlook}

shown by measurements, a relative circle diameter mismatch of only $4 \%$ distorts the minimum such that the fluorescence signal drops by more than $60 \%$ compared to the optimum diameter at a STED power of $230 \mathrm{~mW}$ (Figure 3.4.

The axial-STED OFF-switching pattern suffers from spherical aberrations. Those rise when the refractive index of the immersion medium and sample embedding are unmatched. As a result, the minimum fills up depending on the sample depth, whereby the fluorescence signal is suppressed. Up to a small depth, spherical aberration can be dynamically compensated using the SLM. This allows the use of an objective lens with a high numerical aperture (e.g. oil-immersion, NA=1.4), compared to a water-immersion objective lens (e.g. $\mathrm{NA}=1.2$ ). Without correction of spherical aberration, the maximum imaging depth was less than $\sim 1 \mu \mathrm{m}$ at a STED power of $129 \mathrm{~mW}$. When compensating the rising spherical aberration, the imaging depth was extended to $\sim 3 \mu \mathrm{m}$, which was the deepest structure in this case (Figure 3.6. When using more pixels of the SLM than used in this thesis, compensation in a depth of $\sim 5 \mu \mathrm{m}$ or even more should be possible.

However, imaging of a water-embedded sample with an oil-immersion objective using axial-STED was not possible at depths of more than $\sim 10 \mu \mathrm{m}$ at a moderate STED power of $139 \mathrm{~mW}$ (Figure 3.5). A comparable sample was clearly recorded using a water-immersion objective up to a depth of $180 \mu \mathrm{m}$. In a realistic biological sample random distortions of the wavefront will rise due to the spatially different refractive indices in the sample. Nevertheless, 3D-STED was for the first time demonstrated on a living sample at a maximum depth of $\sim 37 \mu \mathrm{m}$. A resolution along the optical axis of $\sim 153 \mathrm{~nm}$ was shown (Figure 3.7).

With the SLM, oil-immersion and water-immersion objectives can both be used for 3D-STED without adapting the microscope. Without the use of a water-immersion objective no 3D-STED imaging of a living sample at great depth is possible.

\subsection{Intelligent-illumination schemata}

For each set of imaging parameters a maximum fluorescence signal can be achieved, as shown in chapter 2. The STED resolution is limited by the amount of this photon budget. Therefore, several methods have been developed to increase the photon budget.

An effective way to increase the photon budget is to improve the fluorophores by lowering the bleaching cross-section and increasing their brightness [1, 27, 29, 42, 43, 119 121. Beside optimizing the fluorophores, a photobleaching reduction can be realized with sample buffers which lower the oxidation of the fluorophores [88]. Others use unconventional scan schemes to lower the photobleaching, such as fast scanning. The basis of this scanning scheme is to scan a particular 
line (or the whole scan area) very shortly, but multiple times to collect enough fluorescence. Thereby the fluorophores have time to relax from energy states which are connected to photobleaching before they are illuminated again [122] [123]. A similar relaxation is realized by illuminating the fluorophores infrequently, namely T-Rex [86 [124]. When using fluorescent proteins for structure labeling, the bleaching cross-section can be lowered by transfering the proteins at the high-intensity doughnut crest into a state where they almost do not interact with the STED light, while those close to the minimum are imaged, namely MOST [107. Another solution called RESCue lowers the overall light dose by probing during the first fraction of the STED acquisition dwell time if fluorescence is present in an imaged pixel [108]. If not, the illumination is stopped for the rest of the dwell time. A recently published scan scheme makes use of the doughnut-shaped OFFswitching intensity distribution. In MINFIELD scanning, only a small scan area (e.g. $50-200 \mathrm{~nm} S$ side length) is scanned with electro-optical scanners, whereby the high STED intensities are sidestepped to the scan area. The fluorescence signal was improved up to 100 -fold, depending on the scan area size [1].

In this thesis, MINFIELD was realized with galvanometric scanners. A use of those scanners is preferable, since they feature an optical microscope design which allows a greater application range (beside MINFIELD) for biological investigations. A light dose overhead during the relatively slow return motion of the galvanometric scanners was avoided by blocking the beams on the way to the sample during this time. For this purpose, AOMs were used, which can either block the beams or guide those to the sample within a block/rise time of $0.5 \mu \mathrm{s}$. For common MINFIELD imaging parameters, still a little light overhead results from these finite block and rise times of the beams. However, this amount is less than $\sim 0.4 \%$, and has no critical effect on the overall photobleaching. A comparable scan without blocking the beams would lead to a light overhead of $280 \%$ compared to the light dose applied during the actual image acquisition. The beam-blocking in combination with a precise calibration of the galvanometric beam scanners leads to a clear MINFIELD imaging quality which is demonstrated on a variety of biological samples (Figure 4.2 .

MINFIELD was extended to 3D-STED, which is demonstrated on standing DNAbased nanoruler structures with a nominal spot separation of $91 \mathrm{~nm}$ along the optical axis (Figure 4.3). MINFIELD enabled imaging those structures with an isotropic resolution of $\sim 60 \mathrm{~nm}$ and superior fluorescence signal. Without this new illumination concept, the fluorescence photon budget needed for the high resolution and fluorescence signal was not possible.

Dyes and proteins which are used with a STED laser working at a wavelength around $595 \mathrm{~nm}$ (green spectrum) usually have a lower resolution to bleaching rate than the common dyes for a STED laser at $775 \mathrm{~nm}$. Therefore, a reduced bleaching is especially relevant for the green spectrum to attain higher resolutions. Here, the 


\section{Conclusion and Outlook}

green dye Oregon Green 488 is investigated with MINFIELD. In contrast to the expected signal gain by applying MINFIELD, this dye shows a lower fluorescence signal (Figure 4.4). A possible explanation is a higher bleaching in the presence of excitation light and only a low STED intensity (present in the vicinity of the OFF-switching minimum), in respect to areas with higher STED intensities. With only a little STED light the $\mathrm{S}_{1}$ state remains partly populated from where a transition to other energy states is possible, for instance by absorption of another photon within the same pulse or by ISC to the first triplet state. From these states, the intense STED light may cause the main bleaching [82, 85, 89. However, the result can strongly vary with the dye or protein used.

The use of galvanometric scanners allows to implement MINFIELD in a conventional optical STED setup (no electro-optical scanners required) without drawbacks. Thus, researchers can use MINFIELD without building a highly specialized microscope.

The novel adaptive-illumination scan scheme was developed which can significantly lower photobleaching by probing sample structures with a dynamic intensity minimum (DyMIN). Thereby, DyMIN makes use of the doughnut intensity distribution (like MINFIELD) and avoids illumination of sample areas without fluorophores (like RESCue), see figure 5.1. In this first pixel-wise implementation of DyMIN, biological immunofluorescence samples were recorded with more than a 9-fold fluorescence signal increase (Figure 5.6). With DyMIN it is possible to image at high final resolutions while retaining a strong fluorescence signal for reliable structure representation, as demonstrated by acquiring a volume with an isotropic resolution of $\sim 70 \mathrm{~nm}$ FWHM with a single-lens configuration (Figure 5.9). It was demonstrated that DNA-based structures with a spot separation of only $30 \mathrm{~nm}$ in the lateral direction (2D-STED) and a spot separation of $70 \mathrm{~nm}$ in the axial direction (3D-STED) can be resolved with DyMIN. Both feature an unrivaled resolution (FWHM) and fluorescence signal: $\sim 17 \mathrm{~nm}(x, y)$ with $\sim 100$ counts for 2D-STED (Figure 5.7) and $\sim 34 \mathrm{~nm}(z)$ with $\sim 40$ counts for 3D-STED (Figure 5.10).

The best DyMIN imaging parameters were investigated using simulations as well as imaging experiments (Figure 5.5). It was found that DyMIN can significantly lower the light dose on the fluorophores. Thereby, the amount of the light dose reduction strongly depends on the resolutions in the intermediate probing steps. However, with the correct settings, DyMIN counterintuitively reduces the overall light dose by applying more scan steps.

However, the light dose reduction is strongly dependent on the structure and vanishes for large structures. It is evident that sparse structures with small fluorophore clusters perform best. Very sparse structures can also be recorded with MINFIELD with a similar image quality, but the static image size may still lead to unnecessary „empty"imaged pixels (e.g. at the image field corners). With the self-adjusting DyMIN scan, the image boundaries are set as close to the structure as possible, 
allowing for arbitrary shapes. Denser samples can also experience a light dose reduction, as long as any of the DyMIN probe steps has a high enough resolution capable of locating „empty“spaces in the sample (Figure 5.4).

In RESCue STED, the probing for structure is performed with the resolution of the STED image acquisition. The fluorescence signal threshold for probing is only reached if the effective PSF is a little inside the structure (otherwise no signal is present), hence structure further outside with a signal below the threshold (structure rim) is excluded by the RESCue probing. In other words, the probing in RESCue STED cuts off the structure rim. Compared to RESCue STED, the last probing step of DyMIN has a lower resolution compared to the final STED image resolution, and thus does not cut the structure. DyMIN therefore features the advantage to maintain a clearance zone around the actual structure to contain it completely.

Another disadvantage of RESCue is the relatively low fluorescence signal photon flux at high STED resolutions (for which a bleaching reduction becomes important). To achieve a certain reliability of the decision whether a threshold is reached (Poission statistics), a much longer signal integration time with a high STED power (and hence light dose) is needed for RESCue compared to the last intermediate probing step of DyMIN. This means that at high STED resolutions, the RESCue concept becomes more and more inefficient compared to DyMIN, because a high light dose is applied during the longer time necessary to probe for fluorescence.

DyMIN scanning can feature a new level of photon budget, and hence fluorescence signal and resolution in a large scan area.

\subsection{Outlook}

The work includes basic considerations to find the optimum acquisition settings for STED microscopy, depending only on a few parameters. In the future, this knowledge can be used to create an automated optimization routine for the STED imaging parameters, which might require only a few data points at varying dwell times or resolutions. An even more precise model could include the dependence of the excitation on the photobleaching. Also an extension of the model to the green spectrum (including most of the fluorescent proteins) is of great interest, since green dyes have a higher tendency to bleach, and optimized parameters can help to arrive at the best STED images possible.

With the SLM, a superior quality of the OFF-switching minimums for 2D- and axial-STED was attained. The microscope design allows to switch between objective lenses while keeping the best axial-STED performance so that only the SLM phase mask needs to be adapted. An SLM can feature high-quality OFF-switching 


\section{Conclusion and Outlook}

patterns and hence high fluorescence signals and resolutions. In the future, more advanced aberration corrections will allow imaging with superresolved 3D information deep inside living specimens. Therefore, more advanced detection of aberrations and their correction in living samples will push STED even further. Starting from the high fluorescence signal provided by the correct choice of the objective lens, scan techniques like MINFIELD or DyMIN can provide a further image quality enhancement. However, specimen-induced aberrations are often difficult to detect, but some improvements using STED are already possible [58 [125]. Nevertheless, a way to detect aberrations while imaging is an ongoing process and further research is necessary for a routine application.

MINFIELD is a general concept to reduce photobleaching for coordinate-targeted superresolution microscopy, and has only been demonstrated for STED [1]. A MINFIELD-like scan needs to be explored for other superresolution concepts such as ground state depletion (GSD) [126] and RESOLFT [106, 127, 128. For both, a combination of MINFIELD with RESCue [108] or optimization of the sample buffer 88 needs to be explored. The lower photobleaching in MINFIELD may enable to image the same sample structure with different OFF-switching patterns and collect a high fluorescence signal for each. With this high-quality data (high signal-to-noise ratio), a computational fusion of the best resolution of each pattern might be possible, comparable to joint-deconvolution [129] [130 or nonlinear structured illumination microscopy [38]. For this purpose, a better choice for the OFF-switching pattern shapes might exist 96 . Currently, only quadratic-shaped scan areas are used for MINFIELD. A round-shaped scan area will lower the photobleaching, because fewer pixels are scanned and the high doughnut intensities which are guided to the sample while imaging the corners of the quadratic scan area are avoided.

Similar to MINFIELD, a DyMIN-like illumination needs to be explored for other coordinate-targeted superresolution methods like GSD [126 and RESOLFT $106,127,128$.

For all superresolution techniques, the ON/OFF principle is the crucial concept to separate closely spaced fluorophores $15,16,127$. The amount of switching light which is required to separate fluorophores depends on the relative spacing between these. An adaptive-illumination to drive the ON/OFF transfer only as much as possible is a logical procedure for gentle imaging of fluorophores and samples.

The first DyMIN implementation presented here demonstrates a significant bleaching and sample illumination reduction. Nevertheless, the concept is not at the ultimate limit and several improvements are possible. Currently the probing steps have predefined fixed signal integration times. An early illumination stop at any 
probing step is possible if enough fluorescence photons are collected in a fraction of the decision time to make a confident decision on presence or absence of fluorophores.

Currently, all DyMIN probing steps are applied at a pixel if enough fluorescence is detected at each probing step. However, if for instance a large fluorophore cluster does not feature any substructures, the DyMIN steps at higher resolutions will not collect any more information. A registration whether a step at higher resolutions leads to a gain in spatial information can be used to abort later DyMIN steps and save light dose originating from those. It is also possible to apply a RESCue logic to the illumination at the last DyMIN step. This would include aborting the illumination if not enough fluorescence is present at the final resolution, or if already enough fluorescence is collected for a reliable structure definition [108].

Another DyMIN improvement is possible by the use of a more complex scan pattern. Currently, the image is scanned only once in a linear fashion (top to bottom). Scanning the whole field of view with a confocal resolution step first, and then sequentially inform the scan coordinates for the next resolution steps and so on until the maximum resolution is reached, features more image information for the decisions taken at each step. This will also allow to skip „empty“sample regions to save acquisition time. Very fast electro-optical scanners will match the requirements for such an elaborate spatial scan. Those can also be used to lower the bleaching by fast scanning [122 [123].

In the current pixel-wise DyMIN implementation, a non-homogeneous bleaching in the final image may occur, since rows imaged later within a fluorophore cluster are bleached more before readout (Figure $5.12(\mathrm{~A})$ ). A solution which will lower this effect includes scanning the final image (with illumination mask $N$ ) several times with a fraction of the dwell time, and accumulating the fluorescence signal of each scan (similar to the MINFIELD scan scheme [1]). To do so, the illumination mask $N$ should be stored after probing. This avoids the unwanted light dose which would be necessary for repeated probing of the structure at each repetition.

With electro-optical scanners, also scan-patterns may be possible which lead to the same product of the fluorescence level and readout time at each pixel. This would feature a linear representation of the marker density in the sample, which is currently not possible due to the non-homogeneous bleaching. Additionally, when recording the complete field of view with each DyMIN probing step at once, the information of neighboring pixels can be incorporated for pixel decisions (currently each pixel is analyzed independently). The neighborhood information could also reverse wrong decisions taken for specific pixels (e.g. one „low"pixel in an otherwise bright surrounding should be imaged), or could be used for floating decision thresholds to better capture out-of-focus fluorophore clusters, which are otherwise only partly imaged (Figure 5.12 (B)). An intelligent algorithm to capture most of the sample features while lowering the bleaching light dose is of great interest. 


\section{Conclusion and Outlook}

DyMIN can be thought of as a sample-adaptive illumination depending on any signal of the sample. Here, the fluorescence signal was used to dynamically adapt the STED power in order to avoid photobleaching. However, other signals from the sample may be used to detect sample parts which, for instance, absorb STED light and can be avoided by adaptive-illumination. Otherwise, these sample areas would heat up by the intense STED light and destroy their surrounding which thus cannot be imaged.

With the developed microscope and illumination schemata, superior resolution enhancements in all three dimensions became possible for advanced live-cell applications and immunofluorescence imaging while maintaining a high fluorescence signal for a clear structure representation. The new understanding of the best STED imaging parameters is a pathway to better images, and can be used in the future to automatically set the microscope to the optimum imaging conditions for a certain application. Intelligent multi-adaptive illumination scan schemata based on DyMIN will provide further advancements in the future. With those, STED fluorescence nanoscopy will further improve, and reach new levels of 2D- and 3D-resolution for biological investigations. 


\section{Material and Methods}

In this section, the publication "Adaptive-Illumination STED Nanoscopy“, Heine J. et al. PNAS (2017), is partly cited.

\subsection{General statements}

Raw data. All image data is acquired in photon counting mode, without further processing unless otherwise indicated. Lookup tables as indicated, from 0 to maximum counts in the respective image, are used for representation of all image data, if not other stated.

Power stated. Powers stated are powers in the focal plane, measured with a slide power sensor head (S170C Thorlabs). STED laser power measurements were performed at $10 \%$ of the STED power and the maximum power linearly approximated.

\subsection{Sample preparation}

Gold beads (Abberior, Germany) A sample consisting of gold nanoparticles mounted in DPX of nominally $150 \mathrm{~nm}$ size.

Fluorescent nanoassemblies (Bead R, GATTAquant, Germany). A sample of nominally $23 \mathrm{~nm}$ size, containing $\sim 80$ ATTO $647 \mathrm{~N}$ fluorophores with low variability in number.

Crimson fluorescent nanobeads. Beads were diluted from the stock solution in water (1:5000), binding to a cleaned coverslip (\# 1.5, thickness $170 \mu \mathrm{m}$ ) which surface was prepared with undiluted poly-L-lysine for $5 \mathrm{~min}$. The sample was mounted in Mowiol (Abberior, Germany). 


\section{Material and Methods}

Mammalian cell culture and immunolabeling. Vero cells were grown and labelled as described before, [1] using antibodies against NUP153 and Lamin B1 (both Abcam, Cambridge, UK).

Living skin fibroblasts. Live cell imaging was performed using SiR-tubulin (Spirochrome, Stein am Rhein, Switzerland) labelled cultured primary human skin fibroblasts. Labelling was performed according to the protocol given in the paper by Lukinavičius, et al. [42]. In brief, cells were grown over night on coverslips. Then they were labeled using $100 \mathrm{nM}$ SiR tubulin in serum free growth medium for 60 min at cell culture conditions. For imaging the dye containing growth medium was exchanged to colorless DMEMgfp medium (Evrogen, Moscow, Russia). Imaging was performed at ambient temperature.

Xenopus GP210 immunolabeling. The sample preparation followed the protocol of F. Göttfert et. al. 78.

Human immunodeficiency virus (HIV). The sample preparation followed the protocol of J. Hanne et. al. 100. Labeling was performed with silicon-rhodamineclip.

Clathrin immunolabeling. The antibody used was: Rabbit anti-Clathrin (Abcam (ab21679), 1:200 dilution).

Nanopillar 91 nm (custom-supplied, GATTAquant, Germany). A DNA origami template which forms a standing nanopillar on the coverslip, consisting of two ensembles of ATTO647N fluorophores, with their centers separated by a nominal distance of $91 \mathrm{~nm}$ along the optical axis.

Neuronal cell culture and immunolabeling. Cultures of hippocampal neurons were prepared from Wistar rats of mixed sex at postnatal day P0-P1 in accordance with the Animal Welfare Law of the Federal Republic of Germany (Tierschutzgesetz der Bundesrepublik Deutschland, TierSchG) and the Regulation about animals used in experiments (1st August 2013, Tierschutzversuchsverordnung). For the procedure of sacrificing rodents for subsequent preparation of any tissue, all regulations given in $\S 4$ TierSchG were followed. Since sacrificing of animals is not an experiment on animals according to $\S 7$ Abs. 2 Satz 3 TierSchG, no specific authorization or notification was required. Cells were plated on coverslips coated with $100 \mu \mathrm{g} / \mathrm{ml}$ polyornithine (Sigma-Aldrich, cat. P3655) and $1 \mathrm{\mu g} / \mathrm{ml}$ laminin (BD Bioscience, 
cat. 354232). Neuronal cultures were maintained in Neurobasal medium (Gibco, cat. 21103049) supplemented with 2\% B27 serum-free supplement (Gibco, cat. 17504044), $2 \mathrm{mM}$ L-glutamine (Gibco, cat. 25030) and pen/strep (100 units/ml and $100 \mathrm{\mu g} / \mathrm{ml}$, respectively, BiochromAG, cat. A2213). On the day after plating, $5 \mu \mathrm{M}$ cytosine $\beta$-D-arabinofuranoside (Sigma, cat. C1768) was added to the cultures. For immunostainings, cells (20 to 30 days in vitro) were washed with PBS and fixed in $4 \%$ PFA in PBS ( $\mathrm{pH} 7.4$ ) for 20 min at room temperature, quenched with ammonium chloride and glycine (100 mM each) for $5 \mathrm{~min}$, permeabilized with $0.1 \%$ Triton X-100 for another 5 min and blocked in PBS supplemented with 1\% BSA for 30 min. Both the primary and secondary antibody incubations were performed in PBS for 1 hour at room temperature. The antibodies used were: anti-Gephyrin (Synaptic Systems, cat. 147 011, 1:200 dilution), anti-betaII spectrin (BD Biosciences, cat. 612563, 1:200 dilution); sheep anti-mouse (Dianova 515-005-003) was labeled with STAR 635P (Abberior, cat. 1-0101-007-6). Samples were mounted in Mowiol supplemented with DABCO.

Mouse sperm. Mouse spermatozoa were collected in PBS from the epididymis of 12 weeks old CD1 mice and frozen in liquid nitrogen. After thawing, spermatozoa were incubated on poly-L-Lysine (Sigma-Aldrich, cat. P8920) coated coverslips for one hour at $37{ }^{\circ} \mathrm{C}$. Samples were then rinsed in PBS and fixed in cold methanol for 10 min, rehydrated in PBS, and blocked in PBS supplemented with 1\% BSA for $30 \mathrm{~min}$. Both the primary and secondary antibody incubations were performed in PBS for one hour at room temperature. The antibody used were: anti-betaTubulin (Sigma-Aldrich, cat. T5201, 1:200 dilution); sheep anti-mouse (Dianova 515-005-003) was labeled with STAR 635P (Abberior, cat. 1-0101-007-6). Sample was mounted in Mowiol.

Nanopillar $70 \mathrm{~nm}$ (custom-supplied, GATTAquant, Germany). A DNA origami template which forms a standing nanopillar on the coverslip, consisting of two groups of $\sim 11$ ATTO $647 \mathrm{~N}$ fluorophores, with their centers separated by a nominal distance of $70 \mathrm{~nm}$ along the optical axis. One fluorophore group has an axial extent of nominally $\sim 15 \mathrm{~nm}$ and the other one of $\sim 25 \mathrm{~nm}$. Since the binding efficiency is not $100 \%$ some docking site remain randomly unstained (grey marks).

Nanoruler (custom-supplied, GATTAquant, Germany). A DNA origami template, specifying two ensembles of $\sim 15$ ATTO $647 \mathrm{~N}$ fluorophores each, with their centers separated by a nominal distance of $30 \mathrm{~nm}$. According to the manufacturer's instructions, dyes in each of the ensemble occupy an area of $13 \times 20 \mathrm{~nm}^{2}$. DNA nanorulers are based on the DNA origami technology. Base structures are rigid multi helix bundles which offer single stranded extensions (docking sites) to bind 


\section{Material and Methods}

complementary dye-labeled oligos (yellow marks). Since the binding efficiency is not $100 \%$ some docking site remain randomly unstained (grey marks). 


\section{Bibliography}

[1] Fabian Göttfert, Tino Pleiner, Jörn Heine, Volker Westphal, Dirk Görlich, Steffen J. Sahl, and Stefan W. Hell. Strong signal increase in STED fluorescence microscopy by imaging regions of subdiffraction extent. Proceedings of the National Academy of Sciences, page 201621495, 2017.

[2] Jörn Heine, Matthias Reuss, Benjamin Harke, Elisa D'Este, Steffen J. Sahl, and Stefan W. Hell. Adaptive-illumination STED nanoscopy. Proceedings of the National Academy of Sciences, page 201708304, August 2017.

[3] Mariano Bossi, Jonas Fölling, Vladimir N. Belov, Vadim P. Boyarskiy, Rebecca Medda, Alexander Egner, Christian Eggeling, Andreas Schönle, and Stefan W. Hell. Multicolor Far-Field Fluorescence Nanoscopy through Isolated Detection of Distinct Molecular Species. Nano Letters, 8(8):2463-2468, August 2008.

[4] Ilaria Testa, Christian A. Wurm, Rebecca Medda, Ellen Rothermel, Claas von Middendorf, Jonas Fölling, Stefan Jakobs, Andreas Schönle, Stefan W. Hell, and Christian Eggeling. Multicolor Fluorescence Nanoscopy in Fixed and Living Cells by Exciting Conventional Fluorophores with a Single Wavelength. Biophysical Journal, 99(8):2686-2694, October 2010.

[5] Johanna Bückers, Dominik Wildanger, Giuseppe Vicidomini, Lars Kastrup, and Stefan W. Hell. Simultaneous multi-lifetime multi-color STED imaging for colocalization analyses. Optics express, 19(4):3130-3143, 2011.

[6] Gerald Donnert, Jan Keller, Christian A. Wurm, Silvio O. Rizzoli, Volker Westphal, Andreas Schönle, Reinhard Jahn, Stefan Jakobs, Christian Eggeling, and Stefan W. Hell. Two-Color Far-Field Fluorescence Nanoscopy. Biophysical Journal, 92(8):L67-L69, April 2007.

[7] Jan Tønnesen, Fabien Nadrigny, Katrin I. Willig, Roland Wedlich-Söldner, and U. Valentin Nägerl. Two-Color STED Microscopy of Living Synapses Using A Single Laser-Beam Pair. Biophysical Journal, 101(10):2545-2552, November 2011.

[8] Sebastian Schnorrenberg, Tim Grotjohann, Gerd Vorbrüggen, Alf Herzig, Stefan W. Hell, and Stefan Jakobs. In vivo super-resolution RESOLFT microscopy of Drosophila melanogaster. eLife, 5:e15567, 2016. 


\section{BIBLIOGRAPHY}

[9] Dmitri B. Papkovsky, editor. Live Cell Imaging, volume 591 of Methods in Molecular Biology. Humana Press, Totowa, NJ, 2010.

[10] Francesca Bottanelli, Emil B. Kromann, Edward S. Allgeyer, Roman S. Erdmann, Stephanie Wood Baguley, George Sirinakis, Alanna Schepartz, David Baddeley, Derek K. Toomre, James E. Rothman, and Joerg Bewersdorf. Two-colour live-cell nanoscale imaging of intracellular targets. Nature Communications, 7:10778, March 2016.

[11] Roman S. Erdmann, Hideo Takakura, Alexander D. Thompson, Felix RiveraMolina, Edward S. Allgeyer, Joerg Bewersdorf, Derek Toomre, and Alanna Schepartz. Super-Resolution Imaging of the Golgi in Live Cells with a Bioorthogonal Ceramide Probe. Angewandte Chemie International Edition, 53(38):10242-10246, September 2014.

[12] Ernst Abbe. Beiträge zur Theorie des Mikroskops und der mikrokopischen Wahrnehmung. pages $413-468,1873$.

[13] T. Wilson. Resolution and optical sectioning in the confocal microscope: PROPERTIES OF THE FLUORESCENT CONFOCAL MICROSCOPE. Journal of Microscopy, 244(2):113-121, November 2011.

[14] Jaroslav Icha, Michael Weber, Jennifer C. Waters, and Caren Norden. Phototoxicity in live fluorescence microscopy, and how to avoid it. BioEssays, 39(8):1700003, August 2017.

[15] W. Hell Stefan and Jan Wichmann. Breaking the diffraction resolution limit by stimulated emission: stimulated-emission-depletion fluorescence microscopy. Opt. Lett, 19:780-2, 1994.

[16] Stefan W. Hell. Far-Field Optical Nanoscopy. Science, 316(5828):1153-1158, May 2007.

[17] Philippe I.H. Bastiaens and Stefan W. Hell. Light microscopy on the move. Journal of Structural Biology, 147(1):1-2, July 2004.

[18] Stefan W Hell, Marcus Dyba, and Stefan Jakobs. Concepts for nanoscale resolution in fluorescence microscopy. Current Opinion in Neurobiology, 14(5):599-609, October 2004.

[19] Reimer, Ludwig. Scanning electron microscopy: Physics of image formation and microanalysis. Springer, berlin and new york 2nd completely rev. and updated edition edition, 1998.

[20] William, David B. and Carter, C. Barry. Transmission Electron Microscopy. Springer, new york 2nd edition edition, 2009. 
[21] Xiao-chen Bai, Chuangye Yan, Guanghui Yang, Peilong Lu, Dan Ma, Linfeng Sun, Rui Zhou, Sjors H. W. Scheres, and Yigong Shi. An atomic structure of human $\gamma$-secretase. Nature, 525(7568):212-217, August 2015.

[22] Kem A. Sochacki, Andrea M. Dickey, Marie-Paule Strub, and Justin W. Taraska. Endocytic proteins are partitioned at the edge of the clathrin lattice in mammalian cells. Nature Cell Biology, 19(4):352-361, March 2017.

[23] Artuoro M. Baró and Ronald G. Reifenberger. Atomic force microscopy in liquid: Biological applications. Wiley-VCH, Weinheim, Germany, 2nd edition edition, 2012.

[24] Greg Haugstad. Atomic force microscopy. A John Wiley 8 Sons, Inc., Hoboken, 2012.

[25] Vancso, G. Julius and Schönherr, Holger. Scanning Force Microscopy of Polymers. Springer, Berlin and London, 2010.

[26] Paul Davidovits and M. D. Egger. Scanning laser microscope for biological investigations. Applied optics, 10(7):1615-1619, 1971.

[27] Chenguang Wang, Masayasu Taki, Yoshikatsu Sato, Aiko Fukazawa, Tetsuya Higashiyama, and Shigehiro Yamaguchi. Super-Photostable Phosphole-Based Dye for Multiple-Acquisition Stimulated Emission Depletion Imaging. Journal of the American Chemical Society, 139(30):10374-10381, August 2017.

[28] Jonathan B Grimm, Brian P English, Heejun Choi, Anand K Muthusamy, Brian P Mehl, Peng Dong, Timothy A Brown, Jennifer Lippincott-Schwartz, Zhe Liu, Timothée Lionnet, and Luke D Lavis. Bright photoactivatable fluorophores for single-molecule imaging. Nature Methods, October 2016.

[29] Alexey N. Butkevich, Gražvydas Lukinavičius, Elisa D'Este, and Stefan W. Hell. Cell-Permeant Large Stokes Shift Dyes for Transfection-Free Multicolor Nanoscopy. Journal of the American Chemical Society, August 2017.

[30] Colin J. R. Sheppard, Shalin B. Mehta, and Rainer Heintzmann. Superresolution by image scanning microscopy using pixel reassignment. Optics Letters, 38(15):2889, August 2013.

[31] Stephan Roth, Colin JR Sheppard, Kai Wicker, and Rainer Heintzmann. Optical photon reassignment microscopy (OPRA). Optical Nanoscopy, 2(1):5, 2013.

[32] Giulia M.R. De Luca, Ronald M.P. Breedijk, Rick A.J. Brandt, Christiaan H.C. Zeelenberg, Babette E. de Jong, Wendy Timmermans, Leila Nahidi Azar, Ron A. Hoebe, Sjoerd Stallinga, and Erik M.M. Manders. Re-scan 


\section{BIBLIOGRAPHY}

confocal microscopy: scanning twice for better resolution. Biomedical Optics Express, 4(11):2644, November 2013.

[33] Winfried Denk, James H. Strickler, and Watt W. Webb. Two-Photon Laser Scanning Fluorescence Microscopy. Science, (248):73-76, April 1990.

[34] Robert W. Watkins and Channing R. Robertson. A total internal-reflection technique for the examination of protein adsorption. Journal of Biomedical Materials Research Part A, 11(6):915-938, 1977.

[35] Mats GL Gustafsson. Surpassing the lateral resolution limit by a factor of two using structured illumination microscopy. Journal of microscopy, 198(2):8287, 2000.

[36] Frohn, J.T., Knapp, H.F., and Stemmer, A. Proceedings of the National Academy of Sciences of the United States of America. 97(13). 2000.

[37] Mats GL Gustafsson, D. A. Agard, J. W. Sedat, and others. I5m: 3d widefield light microscopy with better than 100nm axial resolution. Journal of microscopy, 195(1):10-16, 1999.

[38] Mats GL Gustafsson. Nonlinear structured-illumination microscopy: widefield fluorescence imaging with theoretically unlimited resolution. Proceedings of the National Academy of Sciences of the United States of America, 102(37):13081-13086, 2005.

[39] Stefan W. Hell, S. Lindek, Christoph Cremer, and Ernst HK Stelzer. Measurement of the 4pi-confocal point spread function proves $75 \mathrm{~nm}$ axial resolution. Applied Physics Letters, 64(11):1335-1337, 1994.

[40] Stefan Hell and Ernst HK Stelzer. Fundamental improvement of resolution with a 4pi-confocal fluorescence microscope using two-photon excitation. Optics Communications, 93(5-6):277-282, 1992.

[41] Bo Huang, Hazen Babcock, and Xiaowei Zhuang. Breaking the Diffraction Barrier: Super-Resolution Imaging of Cells. Cell, 143(7):1047-1058, December 2010.

[42] Gražvydas Lukinavičius, Luc Reymond, Keitaro Umezawa, Olivier Sallin, Elisa D'Este, Fabian Goettfert, Haisen Ta, Stefan W Hell, Yasuteru Urano, and Kai Johnsson. Fluorogenic probes for multicolor imaging in living cells. Journal of the American Chemical Society, July 2016.

[43] Gražvydas Lukinavičius, Luc Reymond, Elisa D'Este, Anastasiya Masharina, Fabian Göttfert, Haisen Ta, Angelika Güther, Mathias Fournier, Stefano Rizzo, Herbert Waldmann, Claudia Blaukopf, Christoph Sommer, Daniel W Gerlich, Hans-Dieter Arndt, Stefan W Hell, and Kai Johnsson. Fluorogenic 
probes for live-cell imaging of the cytoskeleton. Nature Methods, 11(7):731733, May 2014.

[44] Stefan W Hell, Steffen J Sahl, Mark Bates, Xiaowei Zhuang, Rainer Heintzmann, Martin J Booth, Joerg Bewersdorf, Gleb Shtengel, Harald Hess, Philip Tinnefeld, Alf Honigmann, Stefan Jakobs, Ilaria Testa, Laurent Cognet, Brahim Lounis, Helge Ewers, Simon J Davis, Christian Eggeling, David Klenerman, Katrin I Willig, Giuseppe Vicidomini, Marco Castello, Alberto Diaspro, and Thorben Cordes. The 2015 super-resolution microscopy roadmap. Journal of Physics D: Applied Physics, 48(44):443001, November 2015.

[45] Steffen J. Sahl, Stefan W. Hell, and Stefan Jakobs. Fluorescence nanoscopy in cell biology. Nature Reviews Molecular Cell Biology, September 2017.

[46] Francisco Balzarotti, Yvan Eilers, Klaus C. Gwosch, Arvid H. Gynn\a a, Volker Westphal, Fernando D. Stefani, Johan Elf, and Stefan W. Hell. Nanometer resolution imaging and tracking of fluorescent molecules with minimal photon fluxes. arXiv preprint arXiv:1611.03401, 2016.

[47] Benjamin Harke, Jan Keller, Chaitanya K. Ullal, Volker Westphal, Stefan W. Hell, and Andreas Schönle. Resolution Scaling in STED microscopy. Optics Express, 16(6):4154 - 4162, March 2008.

[48] Jan Keller. Optimal de-excitation patterns for RESOLFT-Microscopy. 2006.

[49] Robert Kasper, Benjamin Harke, Carsten Forthmann, Philip Tinnefeld, Stefan W. Hell, and Markus Sauer. Single-Molecule STED Microscopy with Photostable Organic Fluorophores. Small, 6(13):1379-1384, June 2010.

[50] D. D. Boehr, D. McElheny, H. J. Dyson, and P. E. Wright. The Dynamic Energy Landscape of Dihydrofolate Reductase Catalysis. Science, 313(5793):1638-1642, September 2006.

[51] Samuel T. Hess, Thanu P.K. Girirajan, and Michael D. Mason. Ultra-High Resolution Imaging by Fluorescence Photoactivation Localization Microscopy. Biophysical Journal, 91(11):4258-4272, December 2006.

[52] Michael J Rust, Mark Bates, and Xiaowei Zhuang. Sub-diffraction-limit imaging by stochastic optical reconstruction microscopy (STORM). Nature Methods, 3(10):793-796, October 2006.

[53] Photo-Switchable Fluorescent Probes. Multicolor Super-Resolution Imaging with. science, 1146598(1749):317, 2007.

[54] Huang Bo, Wenqin Wang, Mark Bates, and Xiaowei Zhuang. Biomechanical Energy Harvesting: Generating Electricity During Walking with Minimal User Effort. Science, 319(5864):810-813, February 2008. 


\section{BIBLIOGRAPHY}

[55] Sara A Jones, Sang-Hee Shim, Jiang He, and Xiaowei Zhuang. Fast, threedimensional super-resolution imaging of live cells. Nature Methods, 8(6):499505, June 2011.

[56] Andreas Schönle and Stefan W. Hell. Calculation of vectorial threedimensional transfer functions in large-angle focusing systems. JOSA A, 19(10):2121-2126, 2002.

[57] Benjamin Harke, Chaitanya K. Ullal, Jan Keller, and Stefan W. Hell. ThreeDimensional Nanoscopy of Colloidal Crystals. Nano Letters, 8(5):1309-1313, May 2008.

[58] Travis J. Gould, Daniel Burke, Joerg Bewersdorf, and Martin J. Booth. Adaptive optics enables 3d STED microscopy in aberrating specimens. Optics express, 20(19):20998-21009, 2012.

[59] Christian Osseforth, Jeffrey R. Moffitt, Lothar Schermelleh, and Jens Michaelis. Simultaneous dual-color 3d STED microscopy. Optics Express, 22(6):7028, March 2014.

[60] Gael Moneron and Stefan W. Hell. Two-photon excitation STED microscopy. Optics express, 17(17):14567-14573, 2009.

[61] Kevin T. Takasaki, Jun B. Ding, and Bernardo L. Sabatini. Live-Cell Superresolution Imaging by Pulsed STED Two-Photon Excitation Microscopy. Biophysical Journal, 104(4):770-777, February 2013.

[62] Alf Honigmann, Veronika Mueller, Haisen Ta, Andreas Schoenle, Erdinc Sezgin, Stefan W. Hell, and Christian Eggeling. Scanning STED-FCS reveals spatiotemporal heterogeneity of lipid interaction in the plasma membrane of living cells. Nature Communications, 5:5412, November 2014.

[63] Mathias P. Clausen, Erdinc Sezgin, Jorge Bernardino de la Serna, Dominic Waithe, B. Christoffer Lagerholm, and Christian Eggeling. A straightforward approach for gated STED-FCS to investigate lipid membrane dynamics. Methods, 88:67-75, October 2015.

[64] Luca Lanzanò, Lorenzo Scipioni, Melody Di Bona, Paolo Bianchini, Ranieri Bizzarri, Francesco Cardarelli, Alberto Diaspro, and Giuseppe Vicidomini. Measurement of nanoscale three-dimensional diffusion in the interior of living cells by STED-FCS. Nature Communications, 8(1), December 2017.

[65] Giuseppe Vicidomini, Andreas Schönle, Haisen Ta, Kyu Young Han, Gael Moneron, Christian Eggeling, and Stefan W. Hell. STED Nanoscopy with Time-Gated Detection: Theoretical and Experimental Aspects. PLoS ONE, 8(1):e54421, January 2013. 
[66] Katrin I Willig, Benjamin Harke, Rebecca Medda, and Stefan W Hell. STED microscopy with continuous wave beams. Nature Methods, 4(11):915-918, November 2007.

[67] Gael Moneron, Rebecca Medda, Birka Hein, Arnold Giske, Volker Westphal, and Stefan W. Hell. Fast STED microscopy with continuous wave fiber lasers. Optics express, 18(2):1302-1309, 2010.

[68] Benjamin Harke, Jenu Varghese Chacko, Heiko Haschke, Claudio Canale, and Alberto Diaspro. A novel nanoscopic tool by combining AFM with STED microscopy. Optical Nanoscopy, 1(1):3, 2012.

[69] Jenu Varghese Chacko, Claudio Canale, Benjamin Harke, and Alberto Diaspro. Sub-diffraction nano manipulation using STED AFM. PloS one, 8(6):e66608, 2013.

[70] Marcus Dyba and Stefan W. Hell. Focal Spots of Size $\lambda$ / 23 Open Up Far-Field Florescence Microscopy at $33 \mathrm{~nm}$ Axial Resolution. Physical Review Letters, 88(16), April 2002.

[71] Stefan W. Hell, Roman Schmidt, and Alexander Egner. Diffraction-unlimited three-dimensional optical nanoscopy with opposing lenses. Nature Photonics, 3(7):381-387, July 2009.

[72] Dominik Wildanger, Johanna Brückers, Volker Westphal, Stefan W. Hell, and Lars Kastrup. A STED microscope aligned by design. Optics Express, 17(18):16100 - 16110, August 2009.

[73] Pit Bingen, Matthias Reuss, Johann Engelhardt, and Stefan W. Hell. Parallelized STED fluorescence nanoscopy. Optics express, 19(24):2371623726, 2011.

[74] Fabian Manuel Bergermann. Massively parallelized STED nanoscopy. PhD thesis, 2016.

[75] V. Westphal, S. O. Rizzoli, M. A. Lauterbach, D. Kamin, R. Jahn, and S. W. Hell. Video-Rate Far-Field Optical Nanoscopy Dissects Synaptic Vesicle Movement. Science, 320(5873):246-249, April 2008.

[76] Marcel A. Lauterbach, Jan Keller, Andreas Schönle, Dirk Kamin, Volker Westphal, Silvio O. Rizzoli, and Stefan W. Hell. Comparing video-rate STED nanoscopy and confocal microscopy of living neurons. Journal of Biophotonics, 3(7):417-424, April 2010.

[77] Lars Meyer, Dominik Wildanger, Rebecca Medda, Annedore Punge, Silvio O. Rizzoli, Gerald Donnert, and Stefan W. Hell. Dual-Color STED Microscopy at 30-nm Focal-Plane Resolution. Small, 4(8):1095-1100, August 2008. 


\section{BIBLIOGRAPHY}

[78] Fabian Göttfert, Christian A. Wurm, Veronika Mueller, Sebastian Berning, Volker C. Cordes, Alf Honigmann, and Stefan W. Hell. Coaligned DualChannel STED Nanoscopy and Molecular Diffusion Analysis at $20 \mathrm{~nm}$ Resolution. Biophysical Journal, 105(1):L01-L03, July 2013.

[79] Sven C. Sidenstein, Elisa D'Este, Marvin J. Böhm, Johann G. Danzl, Vladimir N. Belov, and Stefan W. Hell. Multicolour Multilevel STED nanoscopy of Actin/Spectrin Organization at Synapses. Scientific Reports, 6:26725, May 2016.

[80] Franziska R. Winter, Maria Loidolt, Volker Westphal, Alexey N. Butkevich, Carola Gregor, Steffen J. Sahl, and Stefan W. Hell. Multicolour nanoscopy of fixed and living cells with a single STED beam and hyperspectral detection. Scientific Reports, 7:46492, April 2017.

[81] Christian Eggeling, Andreas Volkmer, and Claus A. M. Seidel. Molecular Photobleaching Kinetics of Rhodamine $6 \mathrm{~g}$ by One- and Two-Photon Induced Confocal Fluorescence Microscopy. ChemPhysChem, 6(5):791-804, May 2005.

[82] Christian Eggeling, J. Widengren, R. Rigler, and C. A. M. Seidel. Photobleaching of fluorescent dyes under conditions used for single-molecule detection: Evidence of two-step photolysis. Analytical chemistry, 70(13):26512659, 1998.

[83] Christian Ringemann, Andreas Schönle, Arnold Giske, Claas von Middendorff, Stefan W. Hell, and Christian Eggeling. Enhancing Fluorescence Brightness: Effect of Reverse Intersystem Crossing Studied by Fluorescence Fluctuation Spectroscopy. ChemPhysChem, 9(4):612-624, March 2008.

[84] Christian Eggeling, Jerker Widengren, Leif Brand, Jörg Schaffer, Suren Felekyan, and Claus A. M. Seidel. Analysis of Photobleaching in SingleMolecule Multicolor Excitation and Förster Resonance Energy Transfer Measurements ${ }^{\dagger}$. The Journal of Physical Chemistry A, 110(9):2979-2995, March 2006.

[85] Andreas Schönle, Claas Von Middendorff, Christian Ringemann, Stefan W. Hell, and Christian Eggeling. Monitoring triplet state dynamics with fluorescence correlation spectroscopy: Bias and correction: MONITORING DARK STATE DYNAMICS WITH FCS. Microscopy Research and Technique, 77(7):528-536, July 2014.

[86] Gerald Donnert, Christian Eggeling, and Stefan W. Hell. Triplet-relaxation microscopy with bunched pulsed excitation. Photochemical $\&$ Photobiological Sciences, 8(4):481, 2009. 
[87] Gerald Donnert, Christian Eggeling, and Stefan W Hell. Major signal increase in fluorescence microscopy through dark-state relaxation. Nature Methods, 4(1):81-86, January 2007.

[88] Jan Vogelsang, Robert Kasper, Christian Steinhauer, Britta Person, Mike Heilemann, Markus Sauer, and Philip Tinnefeld. A Reducing and Oxidizing System Minimizes Photobleaching and Blinking of Fluorescent Dyes. Angewandte Chemie International Edition, 47(29):5465-5469, July 2008.

[89] Joanna Oracz, Volker Westphal, Czesław Radzewicz, Steffen J. Sahl, and Stefan W. Hell. Photobleaching in STED nanoscopy and its dependence on the photon flux applied for reversible silencing of the fluorophore. Scientific Reports, 7(1), December 2017.

[90] Marcus Dyba and Stefan W. Hell. Photostability of a fluorescent marker under pulsed excited-state depletion through stimulated emission. Applied optics, 42(25):5123-5129, September 2003.

[91] E. Rittweger, B.R. Rankin, V. Westphal, and S.W. Hell. Fluorescence depletion mechanisms in super-resolving STED microscopy. Chemical Physics Letters, 442(4-6):483-487, July 2007.

[92] Silvia Galiani, Benjamin Harke, Giuseppe Vicidomini, Gabriele Lignani, Fabio Benfenati, Alberto Diaspro, and Paolo Bianchini. Strategies to maximize the performance of a STED microscope. Optics express, 20(7):7362-7374, 2012.

[93] Martin O. Lenz, Hugo G. Sinclair, Alexander Savell, James H. Clegg, Alice C. N. Brown, Daniel M. Davis, Chris Dunsby, Mark A. A. Neil, and Paul M. W. French. 3-D stimulated emission depletion microscopy with programmable aberration correction: 3-D STED microscopy with programmable aberration correction. Journal of Biophotonics, 7(1-2):29-36, January 2014.

[94] D. Wildanger, R. Medda, L. Kastrup, and S.W. Hell. A compact STED microscope providing $3 \mathrm{~d}$ nanoscale resolution. Journal of Microscopy, 236(1):35-43, October 2009.

[95] Kohei Otomo, Terumasa Hibi, Yuichi Kozawa, and Tomomi Nemoto. STED microscopy - super-resolution bio-imaging utilizing a stimulated emission depletion. Microscopy, 64(4):227-236, August 2015.

[96] Jan Keller, Andreas Schönle, and Stefan W. Hell. Efficient fluorescence inhibition patterns for RESOLFT mircoscopy. Optics express, 6(15):33613371, 2007. 


\section{BIBLIOGRAPHY}

[97] Alexander Egner and Stefan W. Hell. Aberrations in confocal and multiphoton fluorescence microscopy induced by refractive index mismatch. In Handbook of biological confocal microscopy, pages 404-413. Springer, 2006.

[98] Martin J. Booth, M. A. A. Neil, and T. Wilson. Aberration correction for confocal imaging in refractive-index-mismatched media. Journal of Microscopy, 192(2):90-98, 1998.

[99] Brian R. Patton, Daniel Burke, David Owald, Travis J. Gould, Joerg Bewersdorf, and Martin J. Booth. Three-dimensional STED microscopy of aberrating tissue using dual adaptive optics. Optics Express, 24(8):8862, April 2016 .

[100] Janina Hanne, Fabian Göttfert, Jiří Schimer, Maria Anders-Össwein, Jan Konvalinka, Johann Engelhardt, Barbara Müller, Stefan W. Hell, and HansGeorg Kräusslich. Stimulated Emission Depletion Nanoscopy Reveals TimeCourse of Human Immunodeficiency Virus Proteolytic Maturation. ACS Nano, 10(9):8215-8222, September 2016.

[101] Freed Eric. HIV-1 Gag Proteins: Diverse Functions in the Virus Life Cycle. 1998.

[102] Todd R Graham and Michael M Kozlov. Interplay of proteins and lipids in generating membrane curvature. Current Opinion in Cell Biology, 22(4):430436, August 2010.

[103] Jürgen J. Schmied, Carsten Forthmann, Enrico Pibiri, Birka Lalkens, Philipp Nickels, Tim Liedl, and Philip Tinnefeld. DNA Origami Nanopillars as Standards for Three-Dimensional Superresolution Microscopy. Nano Letters, 13(2):781-785, February 2013.

[104] Thomas A. Klar, Stefan Jakobs, Marcus Dyba, Alexander Egner, and Stefan W. Hell. Fluorescence microscopy with diffraction resolution barrier broken by stimulated emission. Proceedings of the National Academy of Sciences, 97(15):8206-8210, 2000.

[105] Michael Hofmann, Christian Eggeling, Stefan Jakobs, and Stefan W. Hell. Breaking the diffraction barrier in fluorescence microscopy at low light intensities by using reversibly photoswitchable proteins. Proceedings of the National Academy of Sciences of the United States of America, 102(49):1756517569, 2005.

[106] Tim Grotjohann, Ilaria Testa, Marcel Leutenegger, Hannes Bock, Nicolai T. Urban, Flavie Lavoie-Cardinal, Katrin I. Willig, Christian Eggeling, Stefan Jakobs, and Stefan W. Hell. Diffraction-unlimited all-optical imaging and 
writing with a photochromic GFP. Nature, 478(7368):204-208, September 2011.

[107] Johann G. Danzl, Sven C. Sidenstein, Carola Gregor, Nicolai T. Urban, Peter Ilgen, Stefan Jakobs, and Stefan W. Hell. Coordinate-targeted fluorescence nanoscopy with multiple off states. Nature Photonics, 10(2):122-128, January 2016.

[108] Thorsten Staudt, Andreas Engler, Eva Rittweger, Benjamin Harke, Johann Engelhardt, and Stefan W. Hell. Far-Field optical nanoscopy with reduced number of state transition cycles. Opt. Express, 19(6):5644-5657, 2011.

[109] R A Hoebe, C H Van Oven, T W J Gadella, P B Dhonukshe, C J F Van Noorden, and E M M Manders. Controlled light-exposure microscopy reduces photobleaching and phototoxicity in fluorescence live-cell imaging. Nature Biotechnology, 25(2):249-253, February 2007.

[110] K. Xu, G. Zhong, and X. Zhuang. Actin, Spectrin, and Associated Proteins Form a Periodic Cytoskeletal Structure in Axons. Science, 339(6118):452-456, January 2013.

[111] Elisa D’Este, Dirk Kamin, Caroline Velte, Fabian Göttfert, Mikael Simons, and Stefan W. Hell. Subcortical cytoskeleton periodicity throughout the nervous system. Scientific Reports, 6:22741, March 2016.

[112] Adish Dani, Bo Huang, Joseph Bergan, Catherine Dulac, and Xiaowei Zhuang. Superresolution Imaging of Chemical Synapses in the Brain. Neuron, 68(5):843-856, December 2010.

[113] Christian G. Specht, Ignacio Izeddin, Pamela C. Rodriguez, Mohamed El Beheiry, Philippe Rostaing, Xavier Darzacq, Maxime Dahan, and Antoine Triller. Quantitative Nanoscopy of Inhibitory Synapses: Counting Gephyrin Molecules and Receptor Binding Sites. Neuron, 79(2):308-321, July 2013.

[114] Yaron M. Sigal, Colenso M. Speer, Hazen P. Babcock, and Xiaowei Zhuang. Mapping Synaptic Input Fields of Neurons with Super-Resolution Imaging. Cell, 163(2):493-505, October 2015.

[115] Francesca Pennacchietti, Sebastiano Vascon, Thierry Nieus, Christian Rosillo, Sabyasachi Das, Shiva K. Tyagarajan, Alberto Diaspro, Alessio Del Bue, Enrica Maria Petrini, Andrea Barberis, and Francesca Cella Zanacchi. Nanoscale Molecular Reorganization of the Inhibitory Postsynaptic Density Is a Determinant of GABAergic Synaptic Potentiation. The Journal of Neuroscience, 37(7):1747-1756, February 2017. 


\section{BIBLIOGRAPHY}

[116] Julien Caron, Clément Fallet, Jean-Yves Tinevez, Lionel Moisan, L Philippe (Ori) Braitbart, Gabriel Y Sirat, and Spencer L Shorte. Conical diffraction illumination opens the way for low phototoxicity super-resolution imaging. Cell Adhesion 83 Migration, 8(5):430-439, September 2014.

[117] Colton Boudreau, Tse-Luen (Erika) Wee, Yan-Rung (Silvia) Duh, Melissa P. Couto, Kimya H. Ardakani, and Claire M. Brown. Excitation Light Dose Engineering to Reduce Photo-bleaching and Photo-toxicity. Scientific Reports, 6:30892, August 2016 .

[118] Jürgen J Schmied, Mario Raab, Carsten Forthmann, Enrico Pibiri, Bettina Wünsch, Thorben Dammeyer, and Philip Tinnefeld. DNA origami-based standards for quantitative fluorescence microscopy. Nature Protocols, 9(6):1367-1391, May 2014.

[119] Yujia Liu, Yiqing Lu, Xusan Yang, Xianlin Zheng, Shihui Wen, Fan Wang, Xavier Vidal, Jiangbo Zhao, Deming Liu, Zhiguang Zhou, Chenshuo Ma, Jiajia Zhou, James A. Piper, Peng Xi, and Dayong Jin. Amplified stimulated emission in upconversion nanoparticles for super-resolution nanoscopy. Nature, February 2017.

[120] Jun Chu, Younghee Oh, Alex Sens, Niloufar Ataie, Hod Dana, John J Macklin, Tal Laviv, Erik S Welf, Kevin M Dean, Feijie Zhang, Benjamin B Kim, Clement Tran Tang, Michelle Hu, Michelle A Baird, Michael W Davidson, Mark A Kay, Reto Fiolka, Ryohei Yasuda, Douglas S Kim, Ho-Leung Ng, and Michael Z Lin. A bright cyan-excitable orange fluorescent protein facilitates dual-emission microscopy and enhances bioluminescence imaging in vivo. Nature Biotechnology, May 2016.

[121] Emiliano Cortés, Paloma A. Huidobro, Hugo G. Sinclair, Stina Guldbrand, William J. Peveler, Timothy Davies, Simona Parrinello, Frederik Görlitz, Chris Dunsby, Mark A. A. Neil, Yonatan Sivan, Ivan P. Parkin, Paul M. W. French, and Stefan A. Maier. Plasmonic Nanoprobes for Stimulated Emission Depletion Nanoscopy. ACS Nano, November 2016.

[122] Yong Wu, Xundong Wu, Rong Lu, Jin Zhang, Ligia Toro, and Enrico Stefani. Resonant Scanning with Large Field of View Reduces Photobleaching and Enhances Fluorescence Yield in STED Microscopy. Scientific Reports, 5:14766, October 2015.

[123] Jale Schneider, Jasmin Zahn, Marta Maglione, Stephan J Sigrist, Jonas Marquard, Jakub Chojnacki, Hans-Georg Kräusslich, Steffen J Sahl, Johann Engelhardt, and Stefan W Hell. Ultrafast, temporally stochastic STED nanoscopy of millisecond dynamics. Nature Methods, 12(9):827-830, July 2015. 
[124] Gerald Donnert, Christian Eggeling, and Stefan W Hell. Major signal increase in fluorescence microscopy through dark-state relaxation. Nature Methods, 4(1):81-86, January 2007.

[125] Brian Patton, Debora Andrade, Daniel Burke, Joerg Bewersdorf, and Martin Booth. Adaptive super-resolution microscopy for imaging thick samples. SPIE Newsroom, March 2016.

[126] Stefan W. Hell and Matthias Kroug. Ground-state-depletion fluorscence microscopy: A concept for breaking the diffraction resolution limit. Applied Physics B: Lasers and Optics, 60(5):495-497, 1995.

[127] Stefan W Hell. Toward fluorescence nanoscopy. Nature Biotechnology, 21(11):1347-1355, November 2003.

[128] Michael Hofmann. RESOLFT-Mikroskopie mit photoschaltbaren Proteinen. 2007.

[129] Maria Ingaramo, Andrew G. York, Eelco Hoogendoorn, Marten Postma, Hari Shroff, and George H. Patterson. Richardson-Lucy Deconvolution as a General Tool for Combining Images with Complementary Strengths. ChemPhysChem, 15(4):794-800, March 2014.

[130] André Klauss, Marcelle König, and Carsten Hille. Upgrade of a Scanning Confocal Microscope to a Single-Beam Path STED Microscope. PLOS ONE, 10(6):e0130717, June 2015. 



\section{A. Appendix}

\section{A.1. Further information}

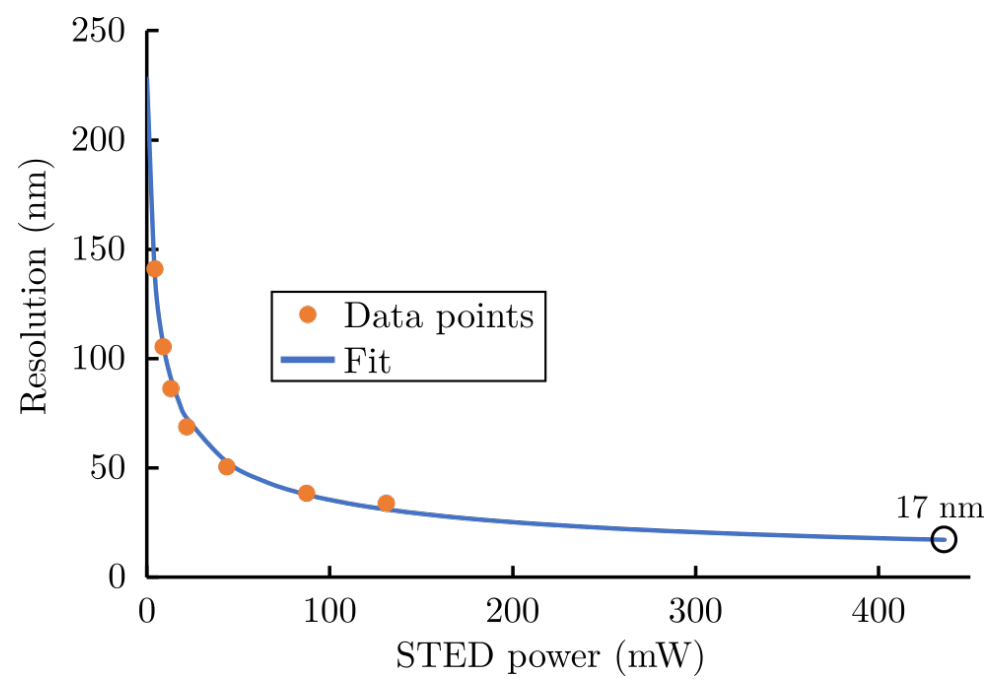

Figure A.1.: Resolution measurement for the used STED microscope. The resolution was measured for different STED powers at the large slope of the resolution curve. The sample consisted of $\sim 23$-nm-sized fluorescent nanoassemblies, containing ATTO 647N (Bead R, GattaQuant). For each data point the resolution of more than 100 isolated beads was evaluated with the software GATTAnalysis v1.2 (GattaQuant). The bead size was taken into account during resolution evaluation. Equation 1.3 was fitted to the data points. At the maximum STED power of $430 \mathrm{~mW}$ in the sample at $40 \mathrm{MHz}$, a resolution (FWHM) of $\sim 17 \mathrm{~nm}$ is expected. 

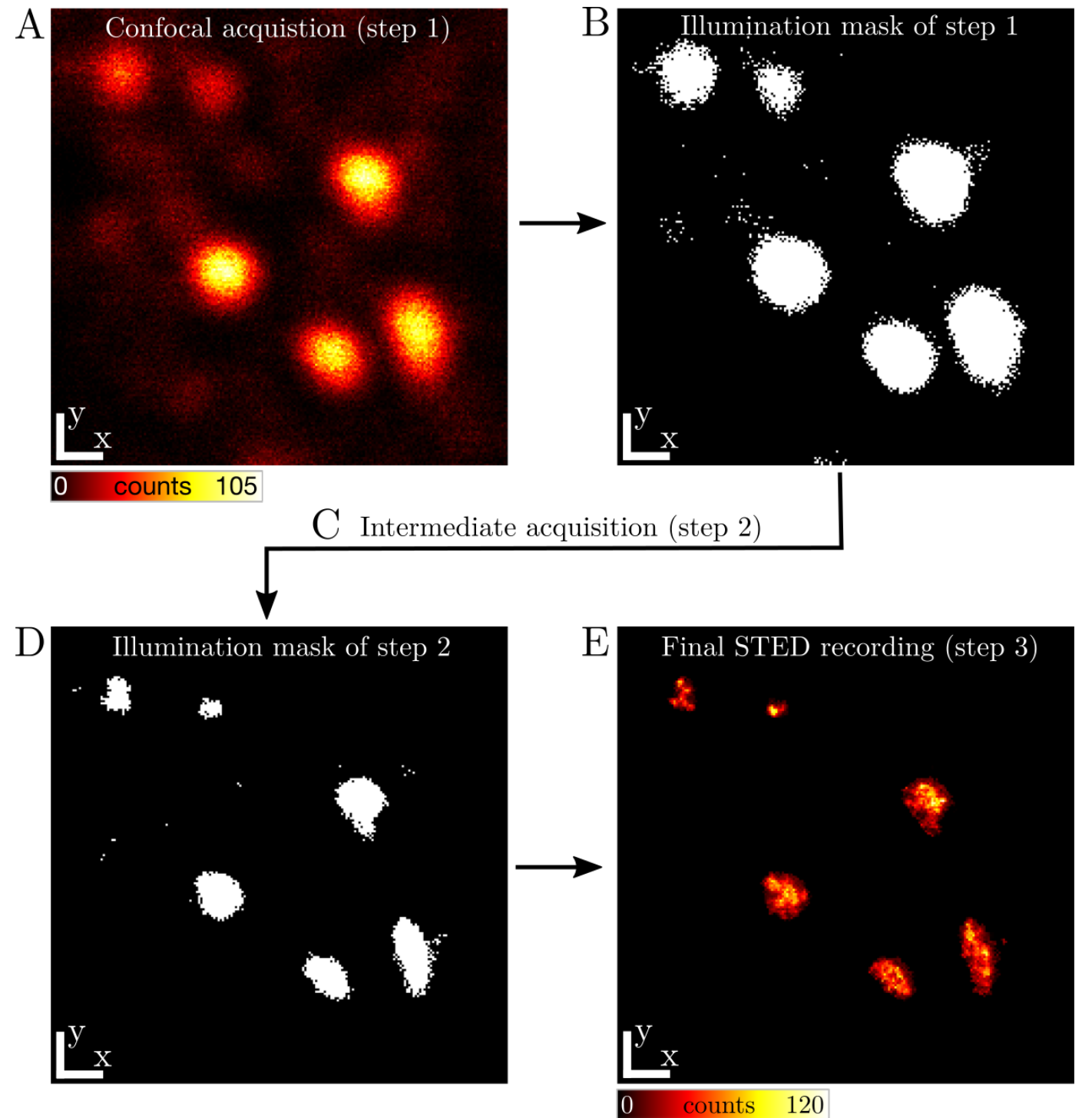

Figure A.2.: Example for a 3-step DyMIN illumination mask creation. Same data are shown as in figure 5.6 in the main text. (A) Step 1 data (confocal) after the complete scan is performed. (B) Illumination mask 1 which is extracted from the data in (A). Black pixels: threshold is not reached, illumination is stopped in these pixels. White pixels: threshold was reached, the illumination continues with the next step. (C) Illumination and readout for step 2 is performed. (D) Illumination mask 2 which originates from step 2 and is the illumination mask for the final step. Same color coding as for (B). (E) Final STED data acquired only in the high pixels (white) of the mask from (D). Please note: the mask creation routine is performed in each pixel (A - E, step 1 - 3) before the scan advances to the next pixel. In other words, the mask creation is not performed in a frame based acquisition mode. Scale bars $(x, y)$ are $200 \mathrm{~nm}$. 

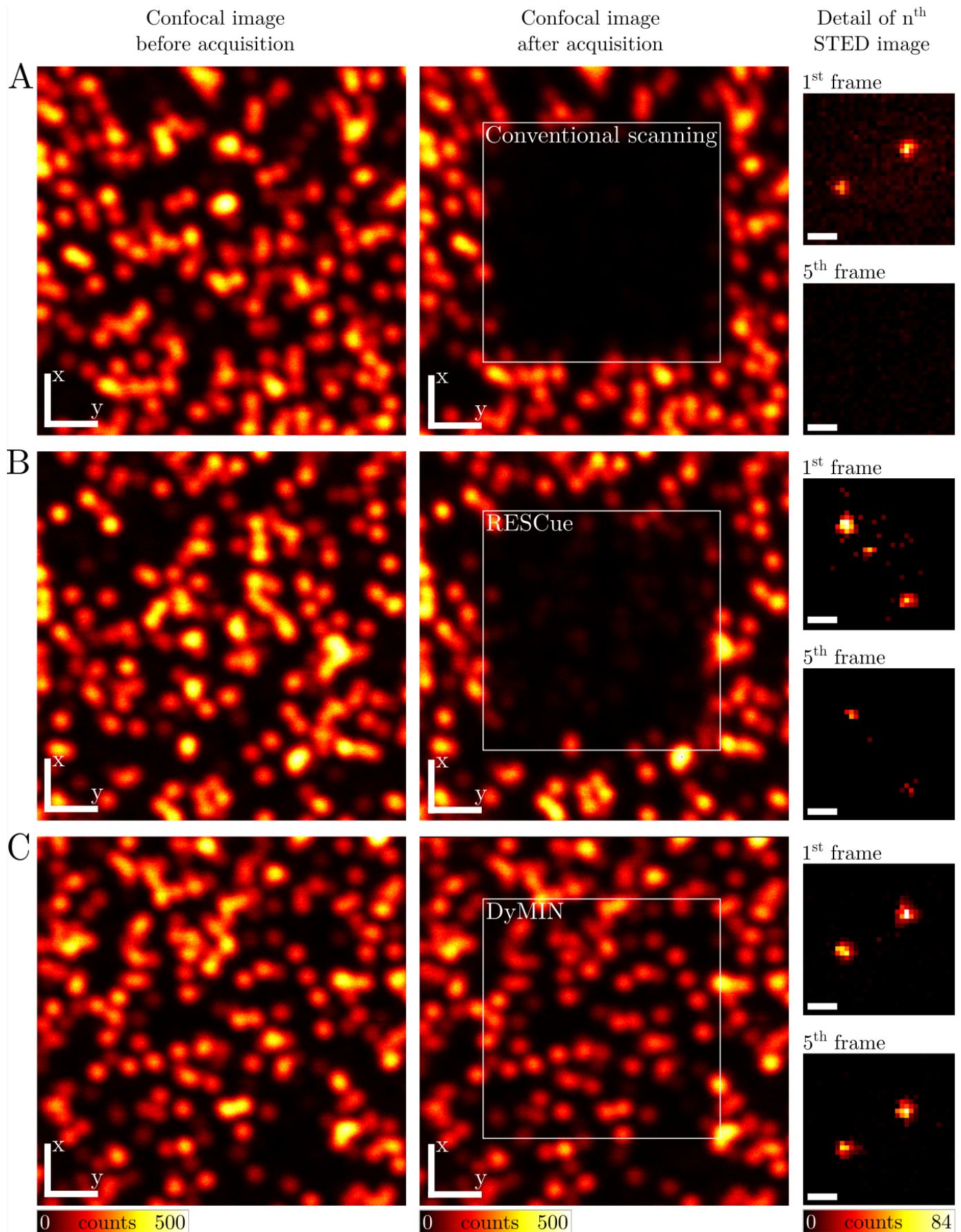

Figure A.3.: Signal reduction over time due to photobleaching for conventional scanning, RESCue and DyMIN (data in Figure 5.5 (C,D). Left column: Confocal fluorescence images before acquisition. Middle column: Confocal fluorescence images after acquisition. Right column: STED image details in $1^{\text {st }}$ and $5^{\text {th }}$ acquired frame out of 20 in total. The sample consisted of dispersed fluorescent nanobeads (Crimson beads, Molecular Probes) of nominally $40 \mathrm{~nm}$ diameter. (A) Conventional scanning, (B) RESCue, (C) DyMIN. Scale bars $(x, y)$ are $1 \mathrm{\mu m}$ (left and middle column), $100 \mathrm{~nm}$ (right column). 

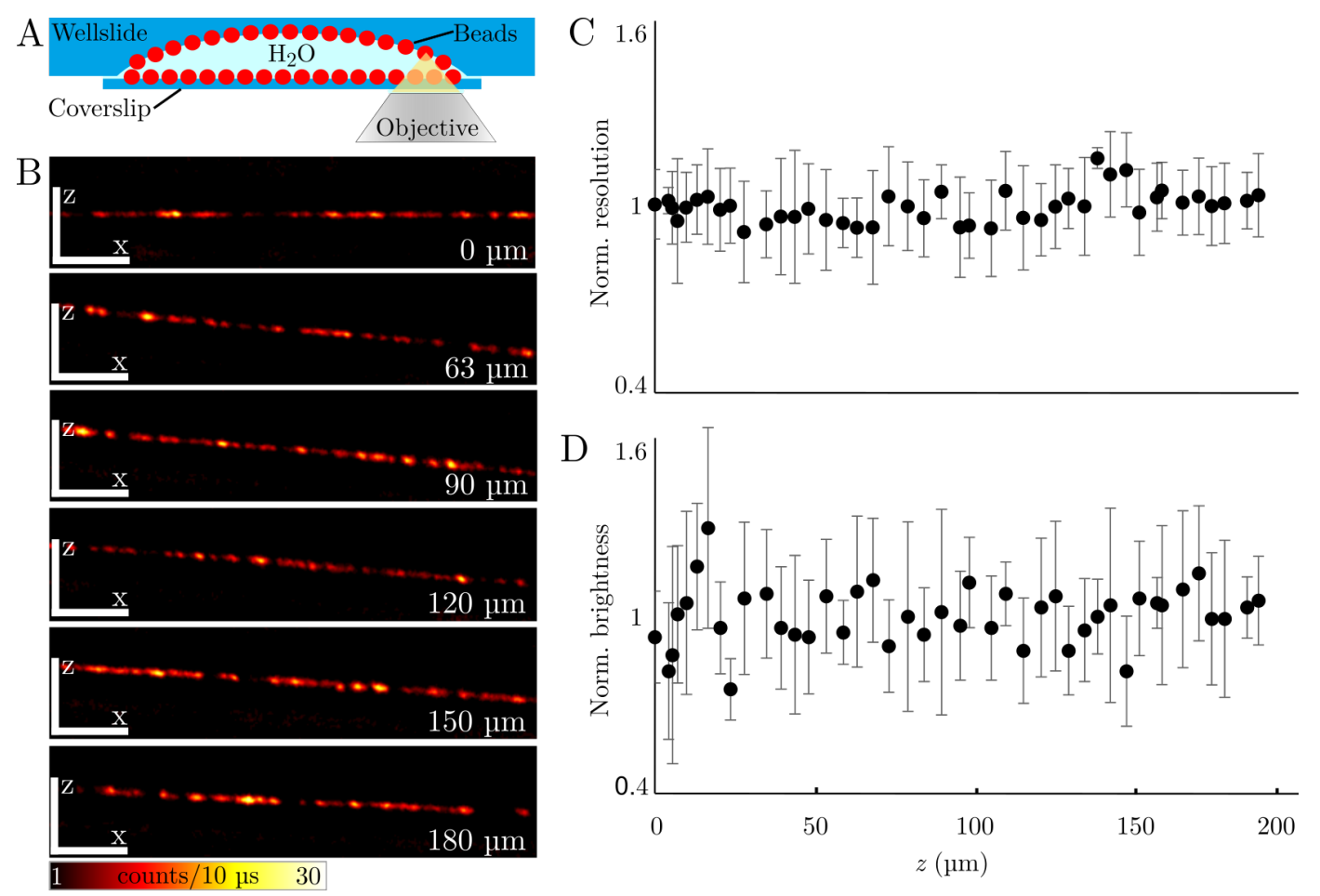

Figure A.4.: Axial-STED with a water-immersion objective in an aqueous mounting media at different depths. (A) Sample schema: $\sim 40 \mathrm{~nm}$-sized fluorescent nanobeads (Crimson, Molecular Probes) are mounted on the surface of a coverslip and on a well slide. The sample was mounted in water, featuring different distances between both bead layers. (B) The axial-STED images of the inside layer for different depth are shown. (C) Measured and normalized resolution of the inside bead layer as a function of the depth. (D) Normalized brightness of the inside bead layer as a function of the depths. Scale bars $(x, z)$ are $400 \mathrm{~nm}(\mathrm{~B})$. 


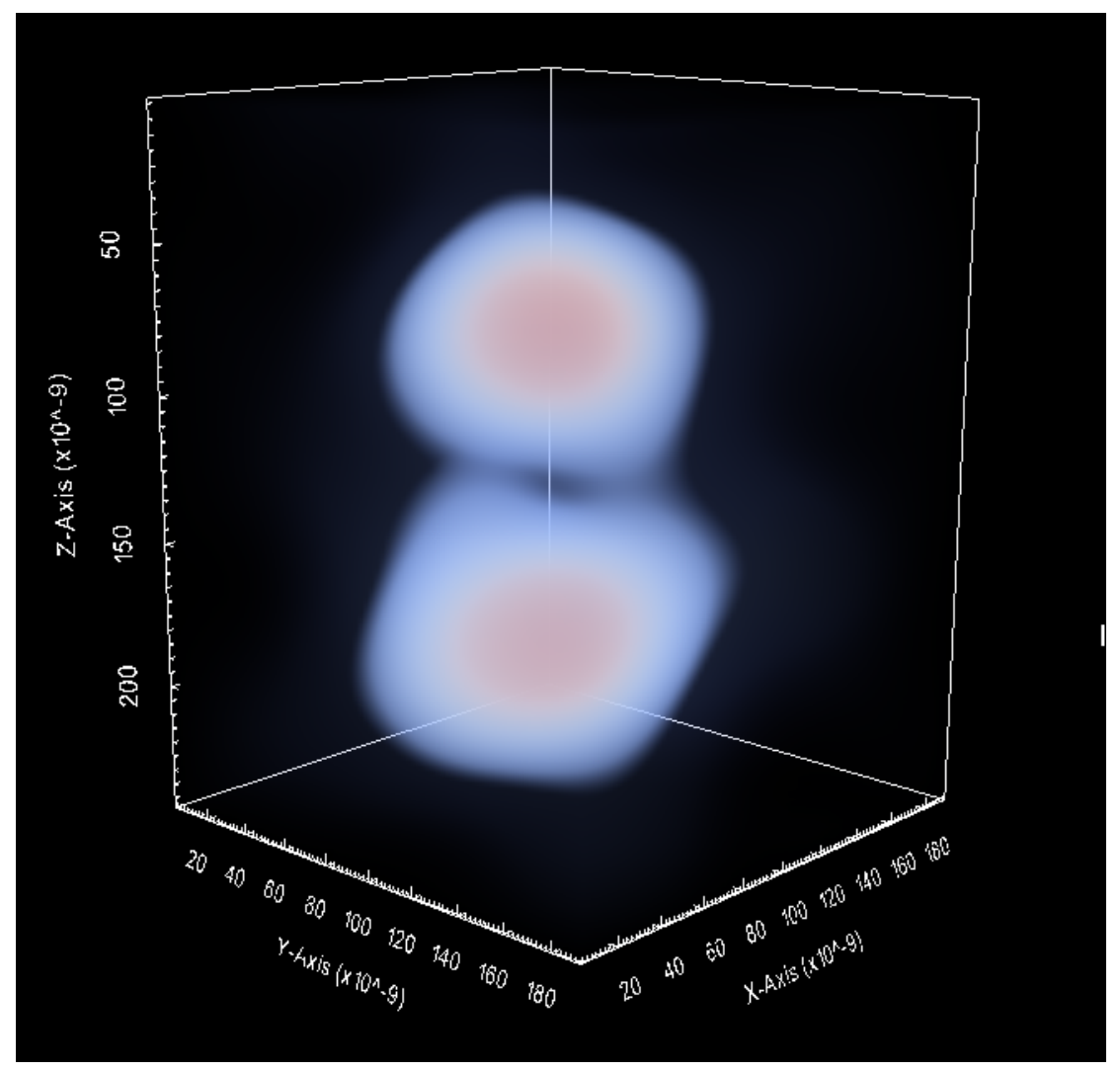

Figure A.5.: 3D rendering of a standing DNA origami with $91 \mathrm{~nm}$ spot separation. A MINFIELD volume was repeatedly scanned and the signal of each individual scan summed up. The volume dimensions are $190 \mathrm{~nm}$ for $x$ and $y$, and $250 \mathrm{~nm}$ for $z$. 


\section{A.2. Imaging parameters}

In the following tables the image parameters used within this thesis are listed.

\begin{tabular}{llllll}
\hline Figure & Measurement & $\begin{array}{l}\text { Excitation } \\
(\mu \mathrm{W})\end{array}$ & $\begin{array}{l}\text { STED } \\
(\mathrm{mW})\end{array}$ & $\begin{array}{l}\text { Pixelsize } \\
(\mathrm{nm})\end{array}$ & $\begin{array}{l}\text { Dwell time } \\
(\mu \mathrm{s})\end{array}$ \\
\hline 2.3 & $40 \mathrm{~nm}$ & 10 & 129 & 15 & varied \\
\hline 2.3 & $45 \mathrm{~nm}$ & 10 & 172 & 17 & varied \\
\hline 3.2 & - & 6.8 & 115 & 20 & 24 \\
\hline 3.4 & - & 13.6 & 230 & 30 & 40 \\
\hline 3.5 & Oil $10 \mu \mathrm{m}$ & 10.7 & 139 & 30 & 20 \\
\hline 3.5 & $\mathrm{H} 2 \mathrm{O} 10 \mu \mathrm{m}$ & 14.9 & 228 & 40 & 20 \\
\hline 3.5 & $\mathrm{H} 2 \mathrm{O} 0-180 \mu \mathrm{m}$ & 3 & 163 & $40 \times 45(x, z)$ & 5 \\
\hline 3.6 & - & 4.6 & 129 & 30 & 40 \\
\hline 3.7 & - & 9.6 & 48 & $30 \times 44(x, z)$ & 21 \\
\hline 4.2 & GP210 & 4.5 & 230 & 8 & $14 \times 30$ \\
\hline 4.2 & HIV & 11.2 & 150 & 5 & $10 \times 30$ \\
4.2 & Clathrin & 9 & 230 & 5 & $10 \mathrm{x} 15$ \\
\hline 4.3 & Nanopillar & 45 & 344 & 10 & $10 \mathrm{x} 10$ \\
\hline 4.4 & - & 1.29 & 19.5 & 5 & $n \times 20$ \\
\hline
\end{tabular}




\begin{tabular}{|c|c|c|c|c|}
\hline Figure 5.2 & Step 1 & Step 2 & Step 3 & Step 4 \\
\hline Excitation $(\mu \mathrm{W})$ & 18 & 18 & 18 & 18 \\
\hline Pixelsize (nm) & \multicolumn{4}{|c|}{15} \\
\hline \multicolumn{5}{|l|}{2 step } \\
\hline Decision/dwell time ( $\mu \mathrm{s})$ & 10 & 100 & - & - \\
\hline STED $(\mathrm{mW})$ & 0 & 103 & - & - \\
\hline DyMIN threshold (counts) & 10 & - & - & - \\
\hline \multicolumn{5}{|l|}{3 step } \\
\hline Decision/dwell time ( $\mu \mathrm{s})$ & 10 & 10 & 100 & - \\
\hline STED $(\mathrm{mW})$ & 0 & 32 & 103 & - \\
\hline DyMIN threshold (counts) & 10 & 16 & - & - \\
\hline \multicolumn{5}{|l|}{4 step } \\
\hline Decision/dwell time ( $\mu \mathrm{s})$ & 10 & 10 & 10 & 100 \\
\hline STED $(\mathrm{mW})$ & 0 & 11 & 64 & 103 \\
\hline DyMIN threshold (counts) & 10 & 20 & 10 & - \\
\hline \begin{tabular}{l|l|l} 
Figure & 5.5 & $\mathrm{~A}$ \\
\end{tabular} & Step 1 & Step 2 & Step 3 & Step 4 \\
\hline Excitation $(\mu \mathrm{W})$ & 18 & 18 & 18 & - \\
\hline STED $(\mathrm{mW})$ & 0 & $0-108$ & $43-185$ & - \\
\hline Decision/dwell time ( $\mu \mathrm{s})$ & 10 & 10 & 100 & - \\
\hline DyMIN threshold (counts) & 10 & 5 & - & - \\
\hline Pixelsize (nm) & & 1 & & \\
\hline
\end{tabular}

\begin{tabular}{|c|c|c|c|c|}
\hline \begin{tabular}{l|l|l} 
Figure & $5.5 \mathrm{~A}$
\end{tabular} & Step 1 & Step 2 & Step 3 & Step 4 \\
\hline Excitation $(\mu \mathrm{W})$ & 1.3 & 7 & 7 & - \\
\hline STED $(\mathrm{mW})$ & 0 & 39 & 215 & - \\
\hline Decision/dwell time ( $\mu \mathrm{s})$ & 10 & 10 & 100 & - \\
\hline DyMIN threshold (counts) & 15 & 10 & - & - \\
\hline Pixelsize (nm) & \multicolumn{4}{|c|}{15} \\
\hline
\end{tabular}




\begin{tabular}{|c|c|c|c|c|}
\hline \begin{tabular}{l|l|l} 
Figure & 5.5 & $\mathrm{C}($ DyMIN)
\end{tabular} & Step 1 & Step 2 & Step 3 & Step 4 \\
\hline Excitation $(\mu \mathrm{W})$ & 9 & 9 & 9 & - \\
\hline STED (mW) & 0 & 65 & 301 & - \\
\hline Decision/dwell time ( $\mu \mathrm{s})$ & 10 & 10 & 100 & - \\
\hline DyMIN threshold (counts) & 10 & 5 & - & - \\
\hline Pixelsize (nm) & \multicolumn{4}{|c|}{15} \\
\hline
\end{tabular}

\begin{tabular}{|c|c|c|c|c|}
\hline \begin{tabular}{l|l|l|} 
Figure & 5.6 & $\mathrm{~A}$
\end{tabular} & Step 1 & Step 2 & Step 3 & Step 4 \\
\hline Excitation $(\mu \mathrm{W})$ & 2.5 & 15 & 15 & - \\
\hline STED $(\mathrm{mW})$ & 0 & 22 & 172 & - \\
\hline Decision/dwell time ( $\mu \mathrm{s})$ & 10 & 10 & 100 & - \\
\hline DyMIN threshold (counts) & 14 & 7 & - & - \\
\hline Pixelsize (nm) & \multicolumn{4}{|c|}{13} \\
\hline
\end{tabular}

\begin{tabular}{llllll}
\hline Figure 5.6 & B & Step 1 & Step 2 & Step 3 & Step 4 \\
\hline Excitation $(\mu \mathrm{W})$ & 2.5 & 15 & 15 & - \\
STED $(\mathrm{mW})$ & 0 & 26 & 258 & - \\
Decision/dwell time $(\mu \mathrm{s})$ & 10 & 10 & 100 & - \\
DyMIN threshold (counts) & 18 & 13 & - & - \\
Pixelsize $(\mathrm{nm})$ & \multicolumn{5}{c}{13} \\
\hline
\end{tabular}

\begin{tabular}{|c|c|c|c|c|}
\hline Figure $5.7 \mathrm{~B}$ & Step 1 & Step 2 & Step 3 & Step 4 \\
\hline Excitation $(\mu \mathrm{W})$ & 12 & 12 & 21 & 21 \\
\hline STED $(\mathrm{mW})$ & 0 & 11 & 64 & 450 \\
\hline Decision/dwell time ( $\mu \mathrm{s})$ & 10 & 10 & 13 & 150 \\
\hline DyMIN threshold (counts) & 10 & 9 & 7 & - \\
\hline Pixelsize (nm) & \multicolumn{4}{|c|}{8} \\
\hline
\end{tabular}




\begin{tabular}{llllll}
\hline Figure 5.8 & B & Step 1 & Step 2 & Step 3 & Step 4 \\
\hline Excitation $(\mu \mathrm{W})$ & 14 & 14 & 14 & - \\
STED $(\mathrm{mW})$ & 0 & $0-180$ & 201 & - \\
Decision/dwell time ( $\mathrm{\mu s})$ & 10 & 20 & 100 & - \\
DyMIN threshold (counts) & 25 & 10 & - & - \\
Pixelsize $(\mathrm{nm})$ & \multicolumn{5}{c}{30} \\
\hline
\end{tabular}

\begin{tabular}{|c|c|c|c|c|}
\hline \begin{tabular}{ll|l} 
Figure & 5.9 & $\mathrm{~B}$
\end{tabular} & Step 1 & Step 2 & Step 3 & Step 4 \\
\hline Excitation $(\mu \mathrm{W})$ & 1 & 9 & 9 & - \\
\hline STED $(\mathrm{mW})$ & 0 & 43 & 215 & - \\
\hline Decision/dwell time ( $\mu \mathrm{s})$ & 10 & 10 & 80 & - \\
\hline DyMIN threshold (counts) & 16 & 9 & - & - \\
\hline Voxelsize (nm) & \multicolumn{4}{|c|}{30} \\
\hline
\end{tabular}

\begin{tabular}{lllll}
\hline Figure 5.10 & Step 1 & Step 2 & Step 3 & Step 4 \\
\hline Excitation $(\mu \mathrm{W})$ & 4.5 & 4.5 & 18 & 18 \\
STED at 20 Mhz $(\mathrm{mW})$ & 0 & 13 & 52 & 322 \\
Decision/dwell time ( $\mathrm{\mu s})$ & 20 & 20 & 20 & 100 \\
DyMIN threshold (counts) & 20 & 10 & 13 & - \\
Pixelsize $(\mathrm{nm})$ & \multicolumn{5}{c}{17} \\
\hline
\end{tabular}

\begin{tabular}{|c|c|c|c|c|}
\hline Figure 5.11 B (lamina) & Step 1 & Step 2 & Step 3 & Step 4 \\
\hline Excitation $(\mu \mathrm{W})$ & 4.5 & 4.5 & 4.5 & - \\
\hline STED (mW) & 0 & 43 & 301 & - \\
\hline Decision/dwell time ( $\mu \mathrm{s})$ & 10 & 17 & 80 & - \\
\hline DyMIN threshold (counts) & 40 & 20 & - & - \\
\hline Pixelsize (nm) & \multicolumn{4}{|c|}{25} \\
\hline
\end{tabular}




\begin{tabular}{|c|c|c|c|c|}
\hline \begin{tabular}{l|l|l} 
Figure & 5.11 & $\mathrm{~B}$ (NPCs)
\end{tabular} & Step 1 & Step 2 & Step 3 & Step 4 \\
\hline Excitation $(\mu \mathrm{W})$ & 3 & 12.5 & 12.5 & - \\
\hline STED (mW) & 0 & 30 & 344 & - \\
\hline Decision/dwell time ( $\mu \mathrm{s})$ & 10 & 10 & 80 & - \\
\hline DyMIN threshold (counts) & 39 & 30 & - & - \\
\hline Pixelsize (nm) & \multicolumn{4}{|c|}{25} \\
\hline
\end{tabular}




\section{List of Figures}

1.1. Simulated intensity distributions for different STED modes and corresponding phase masks. . . . . . . . . . . . 7

1.2. Jablonski diagram of dye energy-levels. . . . . . . . . . . . . . 11

2.1. Bleaching of the fluorescence level in STED imaging. . . . . . . . . . 15

2.2. Resolution improvement by a 10-fold bleaching reduction. . . . . . . 18

2.3. The fluorescence signal in STED imaging in dependence on the resolution and dwell time. . . . . . . . . . . . . . . . . . . . 19

3.1. Schematic representation of the relevant optical and mechanical microscope components. . . . . . . . . . . . . . 22

3.2. Influence of astigmatism on the 2D-STED OFF-switching pattern and fluorescence signal. . . . . . . . . . . . . . . . . 25

3.3. Measured axial-STED OFF-switching pattern shapes in dependence on the phase mask circle diameter. . . . . . . . . . . . . . 27

3.4. Dependence of the fluorescence signal on the phase mask circle diameter. 28

3.5. Objective-lens dependent penetration depths in water embedded samples with axial-STED. . . . . . . . . . . . . . . . 30

3.6. Correction of spherical aberrations increases the penetration depth with axial-STED. . . . . . . . . . . . . . . . . 32

3.7. Imaging of living cells with axial-STED at great depth. . . . . . . . 33

4.1. Galvanometric beam scanner motion and laser control logic for a certain scan range. . . . . . . . . . . . . . . . 37

4.2. 2D-STED MINFIELD with galvanometric scanners. . . . . . . . . 38

4.3. 3D-STED MINFIELD with isotropic resolution. . . . . . . . . . . . . 40

4.4. Bleaching reduction by MINFIELD for Oregon Green 488 on nuclear pore complexes (indirect immunolabeling, NUP153) . . . . . . . . 42

5.1. STED fluorescence nanoscopy with a Dynamic intensity MINimum

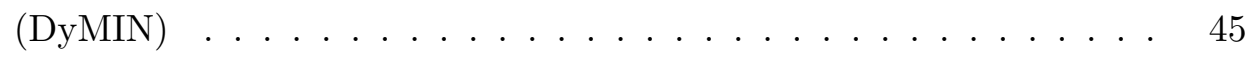

5.2. Influence of the number of intermediate DyMIN steps. . . . . . . . . 51

5.3. Simulated DyMIN scan for different structure geometries. . . . . . . 53

5.4 . Light dose for different scan schemata in dependence on the sample

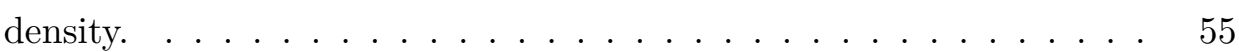


5.5. Reduction of photobleaching by a 3-step DyMIN scan. . . . . . . . . 57

5.6. DyMIN increases the image brightness and structural definition. . . 59

5.7. DyMIN recording with high resolution and fluorescence signal. . . . 61

5.8. Bleaching reduction for 3D-STED with 3-step DyMIN. . . . . . . . . 62

5.9. Lower bleaching by DyMIN enables a volume acquisition with isotropic resolution of tubulin structure in mouse spermatozoa. . . . 64

5.10. DyMIN recording with very 3D-STED resolution. . . . . . . . . . . . 65

5.11. Two-color DyMIN imaging of NPCs and lamina. . . . . . . . . . . 67

5.12. Weaknesses of the here presented pixel-step DyMIN implementation. 69

A.1. Resolution measurement for the used STED microscope. . . . . . . . 99

A.2. Example for a 3-step DyMIN illumination mask creation. . . . . . . 100

A.3. Signal reduction over time due to photobleaching for conventional scanning, RESCue and DyMIN (data in Figure 5.5 (C,D). . . . . . 101

A.4. Axial-STED with a water-immersion objective in an aqueous mounting medium at different depths. . . . . . . . . . . . . . 102

A.5. 3D rendering of an upright DNA origami with $91 \mathrm{~nm}$ spot separation. 103 


\section{List of Tables}

2.1. Effects which lower the obtainable signal in a STED image. . . . . . 14

5.1. Simulation framework to calculate the STED light dose which is applied during DyMIN scanning. . . . . . . . . . . . . . . . . 49

5.2. Resolutions used for figure $[5.2$ for the different DyMIN scans. . . . . 52

\begin{tabular}{|ll|l|l|}
\hline 5.3. & Resolutions for the different DyMIN methods used in figure & 5.4 . . & 56
\end{tabular} 



\title{
Curriculum Vitae
}

\author{
Personal Information
}

$\begin{array}{ll}\text { Name: } & \text { Jörn Heine } \\ \text { Date of Birth: } & \text { 10. August } 1987 \text { in Gifhorn } \\ \text { Nationality } & \text { German }\end{array}$

\section{Academic Career}

Sep. 2008 - Aug. 2011 Study of Physikalische Technologien at the Hochschule für angewandte Wissenschaft und Kunst (HAWK), Göttingen. Finished with degree „Bachelor of Science“.

Sep. 2011 - Oct. 2013 Study of Optical Engineering/Photonics at the Hochschule für angewandte Wissenschaft und Kunst (HAWK), Göttingen. Finished with degree „Master of Science“.

since Jun. 2014

Ph.D student at the Georg-August-University Göttingen.

Research is performed in the company Abberior Instruments GmbH, Göttingen. 


\section{Publications}

\section{Before the Ph.D time}

Heine J., Damm R., Gerhard C., Wieneke S., Viöl W., (2013): Surface activation of plane and curved automotive polymer surfaces by a fittable multi-pin DBD plasma source, Plasma Science and Technology, Vol. 16, No. 6

Gerhard C., Heine J., Bahr B., Brückner,S., Viöl W., (2013): A common-path interferometer including integrated single-prism beam shaping, Measurement, Vol. 46, No. 3

Gerhard C., Heine J., Brückner S., Wienke S., Viöl W., (2013): A hybrid laserplasma ablation method for improved nanosecond laser machining of heavy flint glass, Lasers in Engineering, Vol. 24, Issue 5/6

\section{During the Ph.D time}

Heine J., Reuss M., Harke B., D’Este E., Sahl S., Hell S.W., (2017): Adaptiveillumination STED nanoscopy, Proceedings of the National Academy of Sciences, Vol. 114, No. 37

Göttfert F., Pleiner T., Heine J., Westphal V., Görlich D., Sahl S., Hell S.W., (2017): Strong signal increase in STED fluorescence microscopy by imaging regions of subdiffraction extent, Proceedings of the National Academy of Sciences, Vol. 114, No. 9 


\section{Danksagungen}

Diese Arbeit wurde in der Firma Abberior Instruments GmbH in Göttingen durchgeführt. Viele Personen haben zu dem Erfolg dieser Arbeit beigetragen und deshalb möchte ich ganz herzlich den folgenden Menschen danken:

Prof. Dr. Stefan W. Hell dafür, dass er mir trotz zweiten Bildungswegs die Möglichkeit gegeben hat mich in einen so tollen Wissenschaftsbereich einzubringen, sowie für die Unterstützung, die hilfreichen Diskussionen und die Betreuung dieser Arbeit;

Prof. Dr. Tim Salditt für seine kontinuierliche Unterstützung und die Begutachtung dieser Arbeit;

Dr. Benjamin Harke dafür, dass er mir so viele Dinge beigebracht hat und für die Betreuung dieser Arbeit. Vor allem aber dafür, dass er immer mit Geduld und dem absoluten Willen zu helfen einen Großteil zum erfolgreichen und krisenarmen Abschluss dieser Arbeit beigetragen hat;

Dr. Gerald Donnert für die Finanzierung dieser Arbeit und für die Möglichkeit meine Arbeit in einem so tollen Arbeitsumfeld zu schreiben;

Dr. Christian Wurm für viele hilfreiche Diskussionen, Ideen für Proben, Ratschläge und nette Ausflüge;

Dr. Matthias Reuss, Dr. Lars Kastrup und Dr. Andreas Schönle für die Hilfe bei physikalischen Fragen, elektronischen Entwürfen und Softwareproblemen;

Dr. Steffen Sahl für den lehrreichen Schreibunterricht und die tiefere Vermittlung einer wissenschaftlichen Arbeitsweise; 
Dr. Franziska R. Winter für die tolle Zusammenarbeit im 3D-STED Projekt;

Dr. Elisa D‘Este für die vielen schönen und spannenden Proben und die gemeinsame Arbeit im DyMIN Projekt;

Dr. Jan Keller-Findeisen für die Zusammenarbeit und Simulationen;

Dr. Ellen Rothermel für eine nie versiegende Quelle an Spitzenproben;

Allen Mitarbeitern von Abberior Instruments und Abberior für die vielen Gefallen und die schöne Zeit;

Meiner Schwester für ihre Diskussionen und die Unterstützung;

Und natürlich meinen Eltern für den fortlaufenden Rückhalt und die ständige Hilfsbereitschaft in allen Lebenslagen;

Danke euch allen! 